\title{
Multi-Telescope Radio Observations for Low Frequency Gravitational Wave Astrophysics
}

Megan L. Jones

West Virginia University, mljones1@mix.wvu.edu

Follow this and additional works at: https://researchrepository.wvu.edu/etd

Part of the Cosmology, Relativity, and Gravity Commons, and the Stars, Interstellar Medium and the Galaxy Commons

\section{Recommended Citation}

Jones, Megan L., "Multi-Telescope Radio Observations for Low Frequency Gravitational Wave Astrophysics" (2018). Graduate Theses, Dissertations, and Problem Reports. 3723.

https://researchrepository.wvu.edu/etd/3723

This Dissertation is protected by copyright and/or related rights. It has been brought to you by the The Research Repository @ WVU with permission from the rights-holder(s). You are free to use this Dissertation in any way that is permitted by the copyright and related rights legislation that applies to your use. For other uses you must obtain permission from the rights-holder(s) directly, unless additional rights are indicated by a Creative Commons license in the record and/ or on the work itself. This Dissertation has been accepted for inclusion in WVU Graduate Theses, Dissertations, and Problem Reports collection by an authorized administrator of The Research Repository @ WVU.

For more information, please contact researchrepository@mail.wvu.edu. 


\title{
Multi-Telescope Radio Observations for Low Frequency Gravitational Wave Astrophysics
}

\author{
Megan L. Jones
}

Dissertation Submitted to

The Eberly College of Arts and Sciences at West Virginia University

in partial fulfillment of the requirements

for the degree of

Doctor of Philosophy

in

Physics

\author{
Maura McLaughlin, Ph.D., Chair \\ James M. Cordes, Ph.D. \\ Sarah Burke-Spolaor, Ph.D. \\ Kathryn Williamson, Ph.D. \\ Morgantown, West Virginia, USA \\ 2018
}

Keywords: pulsars, gravitational waves

Copyright 2018 Megan L. Jones 


\title{
Abstract \\ Multi-Telescope Radio Observations for Low Frequency Gravitational Wave Astrophysics
}

\author{
Megan L. Jones
}

The North American Nanohertz Observatory for Gravitational Waves (NANOGrav) has the principal goal of detecting gravitational waves (GWs) in the nanohertz part of the spectrum using pulsar timing. This thesis presents results from radio campaigns at frequencies from $322 \mathrm{MHz}$ to $10 \mathrm{GHz}$ aimed at both multi-messenger constraints on GW sources and improving the timing sensitivity. The primary expected source of GWs at the nanohertz frequencies to which pulsar timing is sensitive are supermassive black hole (SMBH) binaries. We investigate a purported SMBH displaced from the galactic photocenter in NGC 3115. We explore the possibilities that the source is a SMBH binary or a post-merger recoiling SMBH. We place constraints on a possible SMBH companion using observations taken with the NRAO Very Large Array. If a companion SMBH can be confirmed, this system could be a future GW source detectable with pulsar timing.

In order to detect such sources, our pulsar timing array must be as sensitive as possible, requiring the mitigation of all other astrophysical delays, including those from the interstellar medium (ISM). Using NANOGrav wideband multifrequency observations obtained with the Green Bank Telescope and Arecibo Observatory, we characterize frequency-dependent dispersion. This effect is quantified by the dispersion measure (DM). We analyze trends in the DM time series, propose sources of these trends, and identify timescales over which the DM varies beyond measurement errors and therefore can no longer be modeled as constant in timing. Analyzing DM variations aids in characterizing properties of the ISM and informs our timing observation strategy.

Multi-telescope observations around the globe and at complementary frequencies can be used to more sensitively constrain DMs. We compare DMs measured with dual-frequency observations obtained using the Giant Metrewave Radio Telescope (GMRT) to those calculated in the NANOGrav 11-year data release to assess the possible precision of frequency-dependent noise measurements with the GMRT. We discuss the possibility of incorporating the GMRT into international pulsar timing efforts and anticipated challenges in future data combination. 


\section{Acknowledgements}

First, I would like to thank Maura McLaughlin for all of her guidance over the past five years, and for telling me time and time again that everything was going to be alright. I cannot imagine a better or more supportive advisor. I would also like to thank my committee members for their investment in my doctoral success: Jim Cordes for his seemingly infinite wisdom and willingness to share it, Sarah BurkeSpolaor for being so cool and sharing her data wizardry with me, and Kathryn Williamson for helping me build my confidence in front of a crowd and in life.

I would like to thank my undergraduate advisor Eric Wilcots for giving me my start in astronomy research as a plucky teenager, and for helping me find my way both in undergrad and after. Thank you for always giving me good advice, and for guiding me when I could not see the forest through the trees.

Thank you to my numerous colleagues in the NANOGrav collaboration. Among you are my friends, mentors, and role models; it takes a village, and you all are my village. Thank you also to my friends and fellow graduate students at WVU. Grad school would not have been surmountable without our camaraderie and beer therapy. Particular thanks to my best friend Tom, who is always up for long chats, late night proofreading, and who always makes me laugh. A huge thank you to Michael Lam for being my professional and emotional co-pilot, and for keeping me fueled with the best ramen ever while writing this thesis.

Above all, I would like to thank my parents, Gail and Richard, for their unceasing, unwavering, and unconditional support. They taught me to see no limitations and that the stars were (literally) the limit. I would not be the person I am today without their care and guidance. 


\section{Table of Contents}

List of Tables

List of Figures $\quad$ vii

1 Introduction 1

1.1 Gravitational waves . . . . . . . . . . . . . . . . . 3

1.1.1 Gravitational multipoles . . . . . . . . . . . . . . . 4

1.1.2 Sources of gravitational waves . . . . . . . . . . . . . . 6

1.2 Pulsars . . . . . . . . . . . . . . . . . . . . . 10

1.2.1 Millisecond pulsars . . . . . . . . . . . . . . . 10

1.2 .2 Pulsar timing . . . . . . . . . . . . . . . . . 11

1.2.3 Pulsar timing array . . . . . . . . . . . . . . . . 17

1.3 The interstellar medium . . . . . . . . . . . . . . . . . . . . 19

1.3.1 Dispersion . . . . . . . . . . . . . . . . . . 19

1.3.2 Scattering . . . . . . . . . . . . . . 22

1.4 Using multiple radio telescopes for GW astrophysics . . . . . . . . . . 23

1.4.1 Telescopes starting to do PTA science. . . . . . . . . . . 25

1.4.2 Combining timing data . . . . . . . . . . . . . . 26

1.5 Astrophysics across a wide frequency range . . . . . . . . . . . . 26

2 Measurement and Analysis of Variations in Dispersion Measures 28

2.1 Abstract . . . . . . . . . . . . . . . . . . 28

2.2 Introduction . . . . . . . . . . . . . . . . . . . . . . . . . 29

2.3 The NANOGrav 9-year data set . . . . . . . . . . . . . . 31

2.4 Determining significance and trends in the variations . . . . . . . . . 32

2.4.1 Systematic variations . . . . . . . . . . . . . . 33

2.4.2 DM variation timescale . . . . . . . . . . . . . . . 43

2.4.3 Solar-angle correlation ................. . . 44

2.4.4 Pulsar trajectories . . . . . . . . . . . . . . . 45

2.5 Structure functions . . . . . . . . . . . . . . . . . 46

2.6 Results . . . . . . . . . . . . . . . . . . 57

2.6.1 Linear trends and annual periodicities . . . . . . . . . . 57

2.6.2 Structure functions . . . . . . . . . . . . . . . . . 64

2.6.2.1 PSR J1713+0747 . . . . . . . . . . . . 66

2.6.2.2 PSR B1855+09 . . . . . . . . . . . . 67

2.6.2.3 PSR B1937+21 .............. . . 67

2.7 Discussion . . . . . . . . . . . . . . . . . . . . . . . . . . . . . . . . 68

2.8 Acknowledgments . . . . . . . . . . . . . . . . . 71 
3 Investigating the Candidate Displaced AGN in NGC 3115 72

3.1 Abstract . . . . . . . . . . . . . . . 72

3.2 Introduction . . . . . . . . . . . . . . . . . . . 73

3.3 Very Large Array Data . . . . . . . . . . . . . . . . . . . . . . 75

3.4 Analysis of Available Data . . . . . . . . . . . . . . . . . 76

3.4.1 $10 \mathrm{GHz}$ Measurement Results . . . . . . . . . . . 76

3.4.2 Would we have detected a distinct SMBH companion? . . . . 82

3.4.3 Multi-wavelength astrometry . . . . . . . . . . . . . . . 83

3.5 Discussion and Conclusions . . . . . . . . . . . . . . . 87

3.5.1 Where is the radio AGN in NGC 3115? . . . . . . . . . . . 87

3.5.2 Does NGC 3115 contain a binary, offset, or singular central AGN? . . . . . . . . . . . . . . . 87

3.6 Acknowledgments . . . . . . . . . . . . . . . . . . . 88

4 Evaluating Low Frequency Observations at the GMRT 89

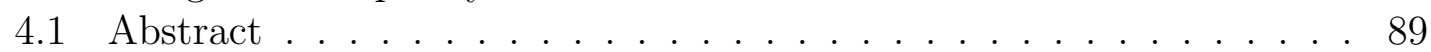

4.2 Introduction . . . . . . . . . . . . . . . . . . 90

4.3 Data . . . . . . . . . . . . . . . . . . . . . . . . . . . . . . . . .

4.4 Comparison of DM measurements . . . . . . . . . . . . . . . . . 101

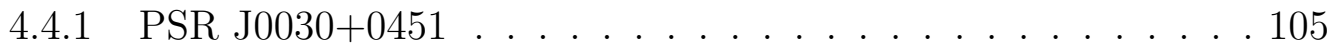

$4.4 .2 \quad$ PSR J1640+2224 . . . . . . . . . . . . . . . . . . . . 105

4.4.3 PSR J1713+0747 . . . . . . . . . . . . . . . 106

4.4.4 PSR J2145-0750 . . . . . . . . . . . . . . . . 107

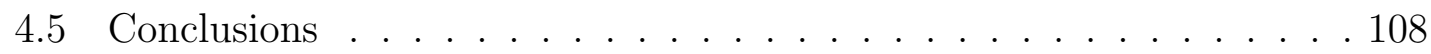

5 Conclusion $\quad 111$

5.1 Importance of DM characterization and understanding the ISM . . . 111

5.2 Investigating a potential SMBH binary candidate . . . . . . . . . . . 112

5.2.1 Data combination and PTA data standards . . . . . . . . 113

5.3 Going forward . . . . . . . . . . . . . . . . . . 115 


\section{List of Tables}

2.1 Properties of NANOGrav MSPs in the 9-Year Data Release. . . . . . 34

2.2 Fitted Trends in the DM Time Series for MSPs in the 9-Year Release 35

2.3 Diffractive timescales for 18 MSPs . . . . . . . . . . . . . 56

2.4 Positions and Corrected Velocities For Three MSPs . . . . . . . . . . 58

2.5 Significance of DM peaks for MSPs within $10^{\circ}$ of the ecliptic . . . . . 62

3.1 Astrometric Position Comparison of Sources at Other Frequencies . . 80

4.1 NANOGrav Observing Frequencies . . . . . . . . . . . . . . . . . . . 98

4.2 GMRT observation lengths . . . . . . . . . . . . . . . . . . . . . . . . . . . . . . . . . . .

4.3 DM estimates using low frequency GMRT data . . . . . . . . . . . . 102

4.4 Sky and system temperatures . . . . . . . . . . . . . . 109 


\section{List of Figures}

1.1 Coverage of experiments across the GW spectrum. . . . . . . . . . . 2

1.2 Polarization of a GW. . . . . . . . . . . . . . . . . 6

1.3 Small galaxies merge to form larger galaxies. . . . . . . . . . . . 8

1.4 The $P-\dot{P}$ diagram. . . . . . . . . . . . . . . . . . . . . . . . . . . . . 12

1.5 Effects on timing residuals due to errors in the timing model. . . . . . 13

1.6 Jitter in simulated pulse profiles. . . . . . . . . . . . . . 15

1.7 Pulse profiles for PSR J1713+0747. . . . . . . . . . . . . . . . 16

1.8 The Hellings-Downs curve. . . . . . . . . . . . . . . . . . . . 18

1.9 Frequency-dependent dispersion. . . . . . . . . . . . . . . . . . . . . . . . . . . . . . 20

1.10 DM variations. . . . . . . . . . . . . . . . . . . . 22

1.11 Pulse scattering. . . . . . . . . . . . . . . . . . . 23

2.1 DM time series for eight pulsars. . . . . . . . . . . . . . . . . 38

2.2 DM time series for eight more pulsars. . . . . . . . . . . . . . . 39

2.3 DM time series for eight more pulsars. . . . . . . . . . . . . . . 40

2.4 DM time series for eight more pulsars. . . . . . . . . . . . . . . . 41

2.5 DM time series for five more pulsars. . . . . . . . . . . . . . . . . 42

2.6 MSP positions with respect to the ecliptic. . . . . . . . . . . . . 44

2.7 DM variations with respect to the solar position angle. . . . . . . . . 45

2.8 MSP trajectories plotted with DM color mapping at each epoch. . . . 47

2.9 MSP trajectories plotted with DM color mapping at each epoch. . . . 48

2.10 MSP trajectories plotted with DM color mapping at each epoch. . . . 49

2.11 MSP trajectories plotted with DM color mapping at each epoch. . . . 50

2.12 Structure functions for the DM variations. . . . . . . . . . . . . 51

2.13 Structure functions for the DM variations. . . . . . . . . . . . . . 52

2.14 Lomb-Scargle periodogram for PSR J0645+5158. . . . . . . . . . . . 58

3.1 Contours of the 10-GHz emission from NGC 3115. . . . . . . . . . . . 77

3.2 Stokes $I$ emission centered on a background source with an X-ray and ugi counterpart. . . . . . . . . . . . . . . . . . . 79

3.3 Flux density with different frequencies. . . . . . . . . . . . . . 81

3.4 The relative positions of the data listed in Table 3.1, with our $10 \mathrm{GHz}$ radio image shown in greyscale. . . . . . . . . . . . . . . . 84

4.1 DM affecting the pulse arrival time for different frequencies. . . . . . 95

4.2 DM comparison from timing. . . . . . . . . . . . . 103 


\section{Chapter 1}

\section{Introduction}

With the detection of gravitational waves (GWs) by the Laser Interferometer Gravitational-Wave Observatory (LIGO), we have entered a new era in GW astronomy. GW astronomy is necessary for studying phenomena only visible through gravitational dynamics and provides a multi-messenger view of processes in the Universe, like supermassive black hole $(\mathrm{SMBH})$ binary mergers. GW frequencies are determined by their sources; for example, SMBH binaries in year orbits emit lowfrequency GWs, while binaries nearing mergers emit much higher frequency GWs. A variety of experiments and observatories are needed to provide coverage across the GW spectrum, seen in Figure 1.1. GW detections in lower frequency regions would be complementary to those already achieved by LIGO and would offer characterization of the GW universe through the observation of a diverse population of sources. A pulsar timing array (PTA), which is made up of an accurately timed network of precise stellar rotators called pulsars, is a low-frequency GW experiment which can be used to look for a correlated signal across multiple pulsars in the network. PTAs are formed by observing pulsars using radio telescopes here on Earth, creating a Galactic scale interferometer.

In this chapter, we will give a brief description of GWs, their detection, sources, as well as pulsar timing and the effects of the interstellar medium. 


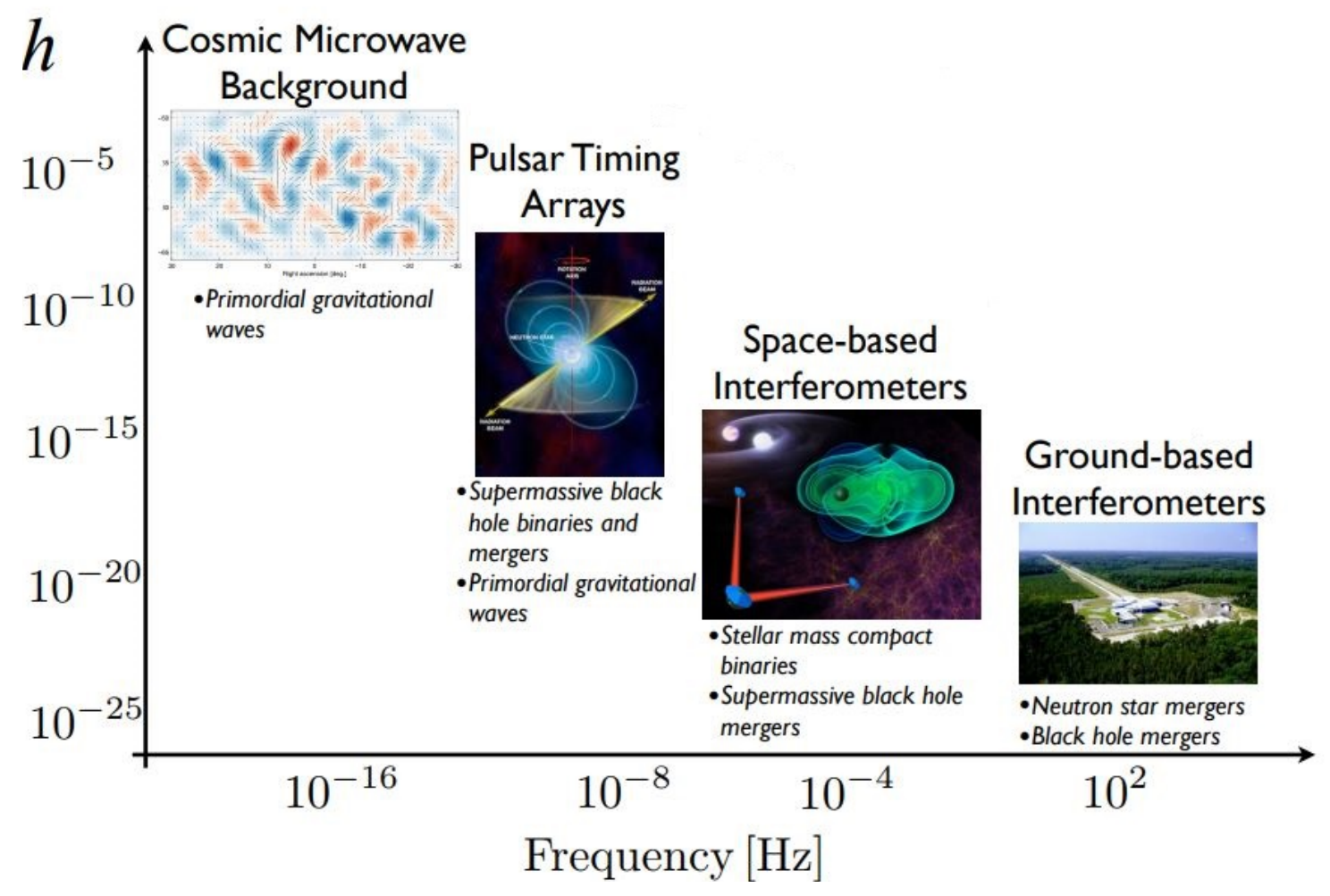

Figure 1.1 Coverage of experiments across the GW spectrum and the detectable sources in each regime. Image credit: NANOGrav. 


\subsection{Gravitational waves}

The need for an improved gravitational theory over the Newtonian description can be seen from looking at how classical mechanics deals with gravitational force

$$
F=\frac{G m_{1} m_{2}}{r^{2}}
$$

where $G$ is the gravitational constant, $m_{1}$ and $m_{2}$ are two masses, and $r$ is the distance between them. Nothing travels faster than light and information is no exception; as can be seen in Eq. 1.1, Newtonian gravity does not incorporate an explicit time dependence and therefore does not account for the time it takes for information to travel. What if the Sun's mass was increasing over time? Equation 1.1 would go from $F \propto m$ to $F(t) \propto m(t)$, which suggests that the planets would feel this change in gravitational force at the same time $t$ (i.e. instantaneously). This would require a speed faster than light and would violate the laws of physics.

The existence of GWs was posited by Einstein in his general theory of relativity (henceforth GR; Einstein, 1915). Contrary to Newton's description of gravity as a force between two objects, Einstein described the influence of gravity as curvature of 4-dimensional spacetime (three spatial dimensions, and one in time); as masses move, the curvature of spacetime changes. GWs are perturbations in spacetime that propagate outward from their source at the speed of light, carrying away energy from the source system along with them.

The effect of GWs traveling through the Universe is to warp the curvature 
of spacetime by contracting or elongating space. If two masses are separated by a distance $L$, the fractional positional perturbation they experience due to a passing GW can be described as

$$
h=\frac{\delta L}{L}
$$

where $\delta L$ is the change in $L$ due to the warping of spacetime. This fractional change $h$ is referred to as the GW strain. GW experiments must be able to very accurately measure $L$ in order to detect the immensely small $\delta L$ produced by GWs. The detection of $\delta L$ and the precision of that measurement are determined by the noise both intrinsic and extrinsic to the detector. It is therefore paramount for GW experiments to characterize sources of noise that may be present in the data.

\subsubsection{Gravitational multipoles}

While any object with mass will have a gravitational field (and will therefore warp spacetime), only certain kinds of systems will emit GWs. In order to determine which systems will be GW emitters, we must be able to characterize gravitational systems, how their mass is distributed, and how they move. It is useful to make a comparison to electromagnetism in order to describe the different properties of massive systems.

The gravitational monopole (the zeroth order term in the gravitational multipole expansion) describes the total mass in a system and does not determine the emission of gravitational waves. A gravitational dipole describes how the mass is distributed. Similar to the electric dipole, the gravitational dipole can be described 


$$
\mathbf{d}=\sum_{i} m_{i} \mathbf{x}_{i}
$$

where $\mathbf{x}_{i}$ is the position vector between the two ends of the dipole. When taking the first derivative of this moment, we get the momentum

$$
\mathbf{p}=\dot{\mathbf{d}}=\sum_{i} m_{i} \dot{\mathbf{x}}_{i}
$$

GWs carry energy away from the system and momentum must be conserved, therefore gravitational dipoles also do not emit gravitational waves.

The gravitational quadrupole moment describes how masses move; it can be calculated as

$$
\mathbf{q}=\sum_{i, j}\left(m_{i}+m_{j}\right) \mathbf{x}_{i} \mathbf{x}_{j}
$$

For instance, a perfect non-rotating sphere moving through space has a constant quadrupole moment because from the object's reference frame, it is at rest. In the case of a binary neutron star system however, regardless of which reference frame is used at least one of the stars is always going to be moving, giving the system a quadrupole moment that changes in time. Therefore the gravitational quadrupole is the first possible nonzero response and is the lowest order contributor to gravitational radiation.

The quadrupolar nature of GWs also determines the shape of the perturbation. A GW affects spacetime by contracting or lengthening space; the directions along which space is affected in either direction depend on the polarization of the GW. 


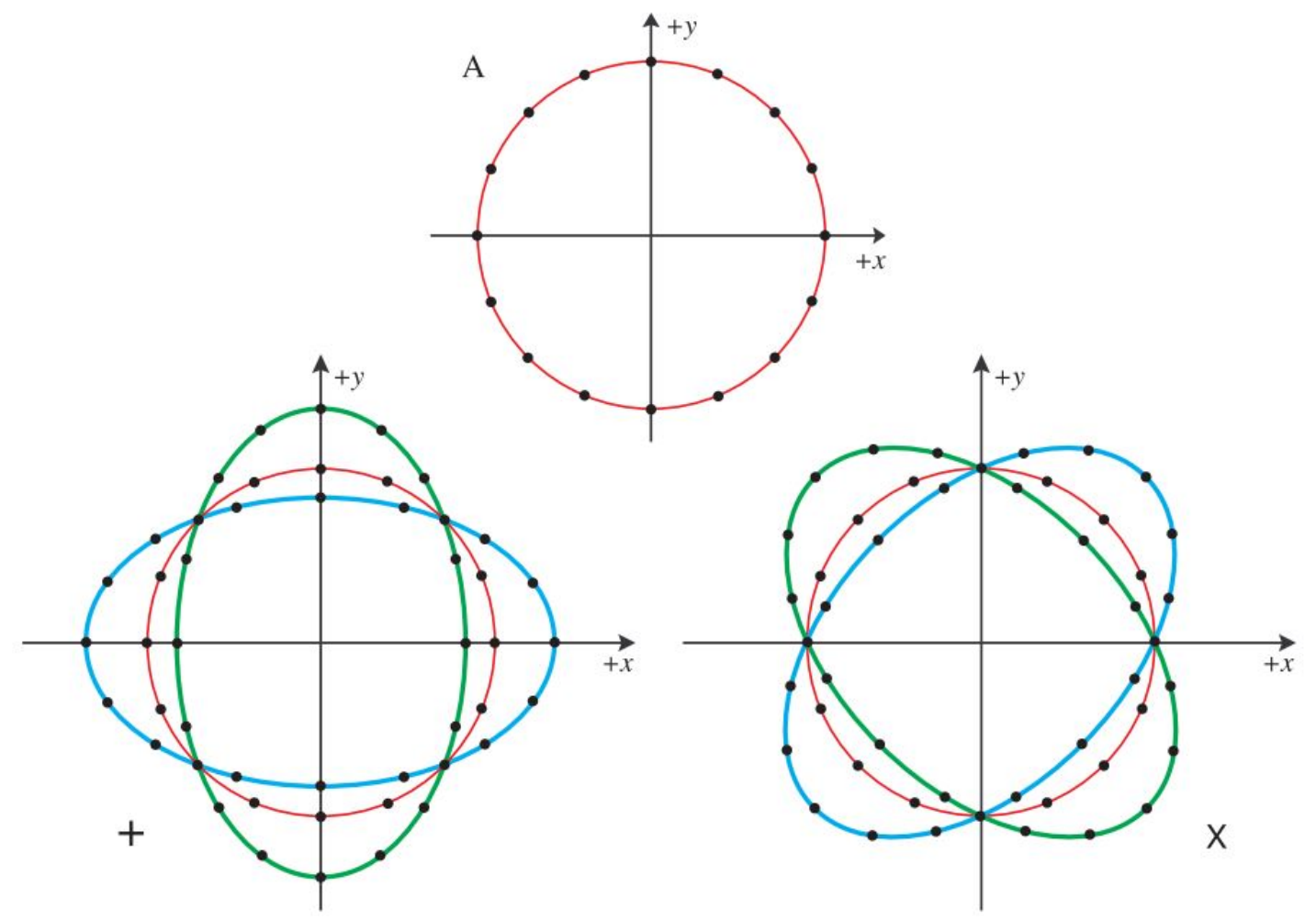

Figure 1.2 A GW affects spacetime by contracting or lengthening space; the directions along which space is affected in either direction depends on the polarization of the GW. Plot A on top shows a ring of test particles unaffected by a GW. The two plots on the bottom show how the ring of test particles will behave if a GW goes through the paper. The ring of test particles initially are perturbed into stretching along the $\mathrm{y}$-axis and contracting along the x-axis (shown in green), then lengthens and contracts in the other directions a half cycle later (shown in blue). Image from Wheeler (2013).

An example of GW polarizations can be seen in Figure 1.2.

\subsubsection{Sources of gravitational waves}

With a decade length data span and weekly to monthly observations, PTAs are sensitive to GW frequencies in the nanohertz to microhertz part of the spectrum. This portion of the GW spectrum includes continuous waves due to supermassive black hole (SMBH) binaries, the stochastic GW background made from many GW 
sources in the Universe, cosmic strings, and maybe even early Universe inflation (shown in Figure 1.1). Unlike electromagnetic radiation, GWs propagate through the Universe virtually unimpeded by matter, rippling the space around them as they pass.

It is widely thought that most galaxies (if not all) host a SMBH at their cores; even our own Milky Way is host to a SMBH. There are several theories on the formation of SMBHs; they are even seen in the somewhat early universe (Volonteri, 2010). Galaxies grow when smaller galaxies merge and form larger galaxies; galaxy mergers also trigger large amounts of star formation. Once black holes are formed through the death of high mass stars, they grow via accretion of matter. As a black hole grows and interacts with more matter in the galaxy, it will move towards the center of the gravitational potential well (i.e. the galactic center). SMBH binaries form following major galaxy mergers. When two galaxies merge, the central SMBHs are brought together into a binary orbit through dynamical friction; as more material is consumed or ejected from the environment around this system, the binary becomes tighter and tighter.

It is at this distance that now, due to the lack of available matter left to interact with, the merging pair can stall. This is known as the final parsec problem. At first glance, the emission of GWs may appear to be a solution to this problem; GW emission results in energy being carried out of the binary system, and with this energy loss, the potential energy of the system decreases, causing the binary orbit to decay and the orbital distance to decrease. However, GW emission does not occur until the SMBHs are much closer together $\left(\sim 10^{-2} \mathrm{pc}\right)$. There is therefore a 


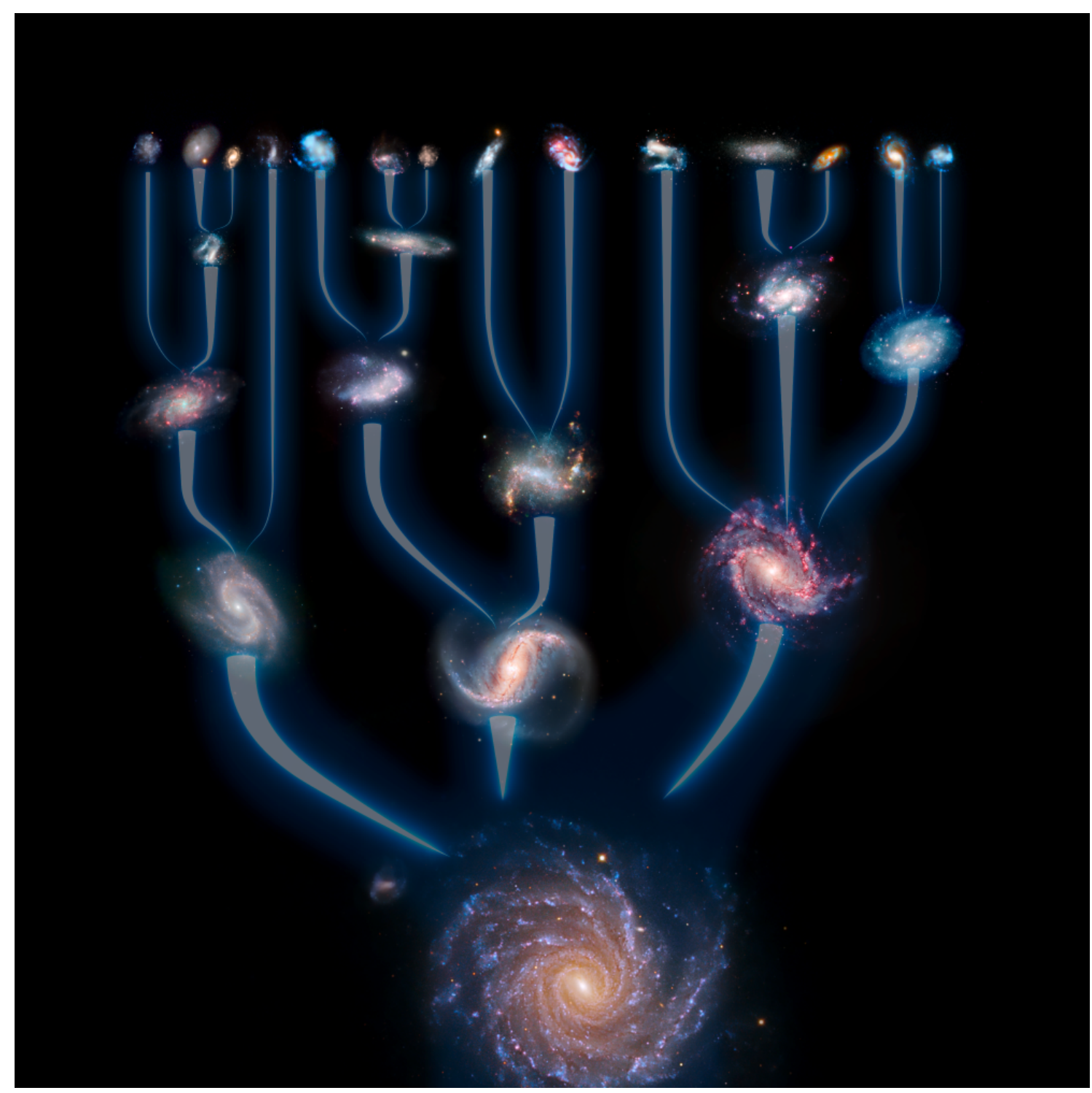

Figure 1.3 Many smaller galaxies merge to form larger, more organized galaxies. This process continues, forming larger, more massive galaxies. Through merger dynamics, the central black holes in these galaxies grow as they absorb newly nearby material as well as other black holes. Image credit: ESO/L. Calada. 
large amount of uncertainty regarding the $\mathrm{SMBH}$ merger rate due to the last parsec problem.

The expected strain from a SMBH binary in a circular orbit is

$$
h \simeq 10^{-17} M_{8}^{5 / 3} f_{y r^{-1}}^{2 / 3} D_{G l y}^{-1} \frac{q}{(1+q)^{2}},
$$

where $M$ is the mass of the binary in units of $10^{8} \mathrm{M}_{\odot}, D$ is the distance to the binary, $f$ is the frequency of the gravitational waves, and $q$ is the mass ratio of the binary. As can be seen from Eq. 1.6, given a detectable threshold value for $h$, then the detection of lower frequency GWs requires higher mass black holes and/or binaries that are closer to the Earth. The detection of GWs produced by SMBH mergers will inform on the percentage of galaxy mergers that eventually produce SMBH mergers, as well as provide conclusive evidence that the final parsec problem can indeed be solved.

The time to merger for a SMBH binary is

$$
\tau=10^{6} M_{8}^{-5 / 3} f_{y r^{-1}}^{-8 / 3} \frac{(1+q)^{2}}{q} \mathrm{yr} .
$$

The stochastic background spectrum is expected to follow an $f^{-2 / 3}$ power law in the case of GW-only driven mergers. SMBH binaries with lower frequencies take more time to merge than those at higher frequencies, therefore low-frequency SMBH binaries have longer lifetimes. Because of this, the gravitational stochastic background is dominated by $\mathrm{SMBH}$ binaries with low orbital frequencies. 


\subsection{Pulsars}

The first pulsar was discovered by graduate student Jocelyn Bell Burnell in 1967 (Hewish et al., 1968). Formed as leftover stellar cores following supernovae, pulsars are highly magnetized, rapidly rotating neutron stars. Charged particles are accelerated along the magnetic field lines of the neutron star; these particles create a beam of electromagnetic radiation as the pulsar spins. As the beam sweeps through space, scientists can detect the beam as a pulse of radiation (hence the name pulsar). The Nobel Prize was later awarded in 1974 (but not to Bell Burnell) for the discovery of pulsars.

Pulsar studies have made their way to the forefront of some of the most groundbreaking astrophysical science. The first exoplanets were discovered orbiting a pulsar, with the Wolszczan \& Frail (1992) discovery of a multi-planet system around the millisecond pulsar PSR 1257+12. The first binary pulsar PSR B1913+16 discovered by Hulse \& Taylor (1975) earned them a Nobel Prize in 1993. It was found that over time, this binary is losing angular momentum and the orbital separation is shrinking (reviewed in Weisberg et al., 2010). This loss is consistent with predictions from general relativity (GR), providing indirect evidence of GW emission.

\subsubsection{Millisecond pulsars}

Pulsars spin with periods ranging from a few tens of seconds to $\sim 1$ millisecond. The first millisecond pulsar (MSP) B1937+21 was detected by Backer et al. (1982) and was determined to have a period of $1.558 \mathrm{~ms}$. MSPs are defined as having 
rotational periods less than $\sim 30$ ms; these short periods are a result of the pulsars having been "recycled" or spun up after accreting material from a binary companion. As a result of this recycling, MSPs have lower magnetic fields and are more precise rotators than their slower canonical counterparts due to their lower spin-down rates, making them excellent tools for precision timing. Properties of different types of pulsars can be seen in the $P-\dot{P}$ diagram in Figure 1.4. According to the ATNF Pulsar Catalogue $\left(\mathrm{PSRCAT}^{1}\right), \sim 2600$ pulsars have been found to date, of which 〜350 are MSPs (Manchester et al., 2005).

\subsubsection{Pulsar timing}

As mentioned earlier, a GW causes a change in the length of the detector (in this case, the light travel time of the pulse). This difference in light travel time causes a change in the time of arrival (TOA) of the pulse, as a different distance means a different light travel time. GWs of the amplitude expected from SMBH binaries likely cause the TOA to vary by a few tens of nanoseconds. As a result, high precision timing is necessary in order to detect this incredibly small change in light travel time.

Due to the stability of MSP rotation periods, pulse TOAs can be predicted through pulsar timing and the determination of a timing model. A basic timing model consists of a Taylor expansion of the rotation phase $\phi$ around some initial

\footnotetext{
${ }^{1}$ http://www.atnf.csiro.au/people/pulsar/psrcat/
} 


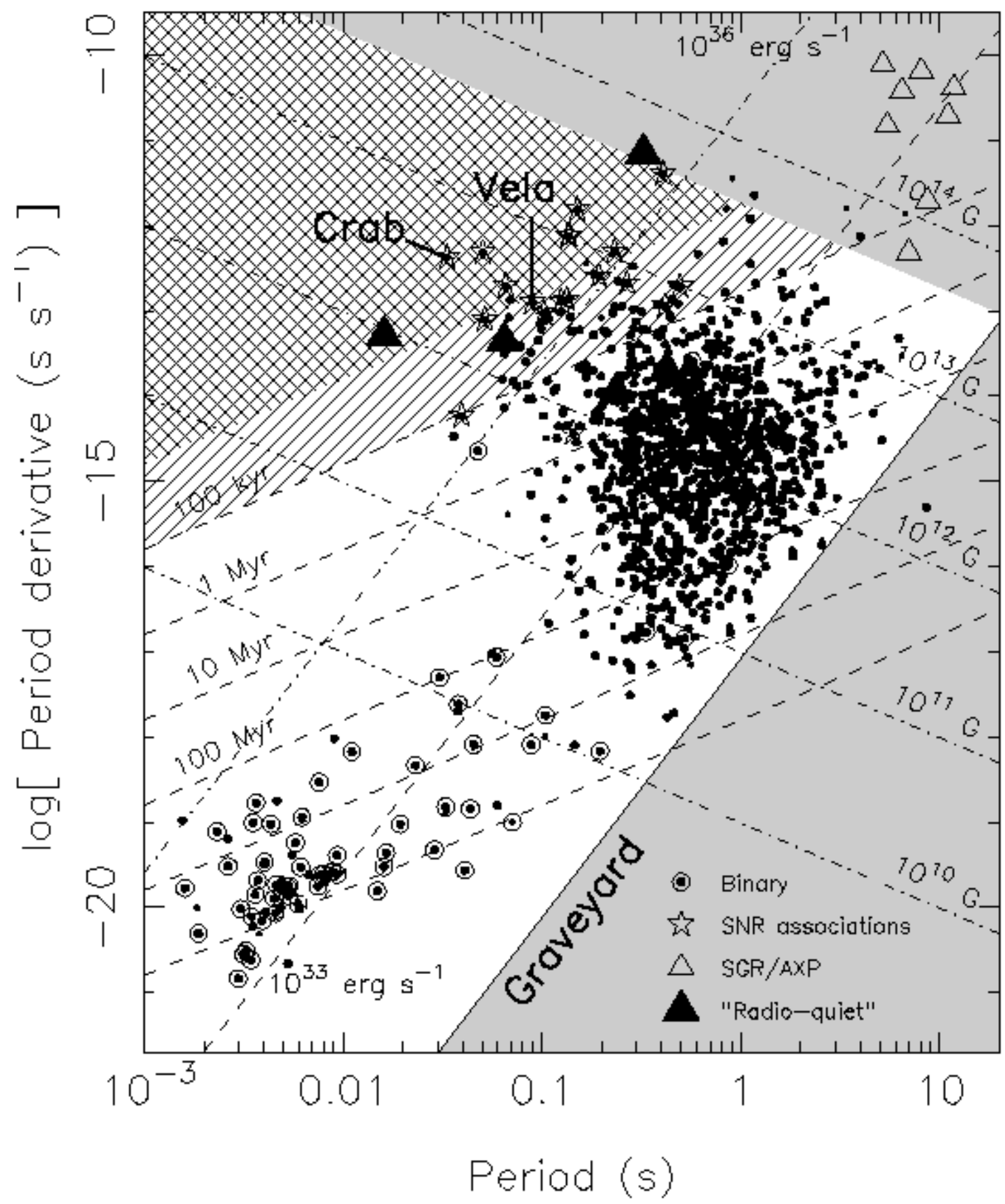

Figure 1.4 The $P-\dot{P}$ diagram. Lines indicate constant characteristic ages, magnetic fields, and spin-down luminosity. The grey region shows forbidden regions for the pulsar population. Most of the MSPs plotted above are in binary orbits; the short periods of these pulsars are a result of them having been spun up after accreting material from their binary companion. The MSP population has smaller period derivatives compared to their spin period, and therefore make more stable rotators than canonical pulsars. Plot taken from Handbook of Pulsar Astronomy (Lorimer \& Kramer, 2012). 

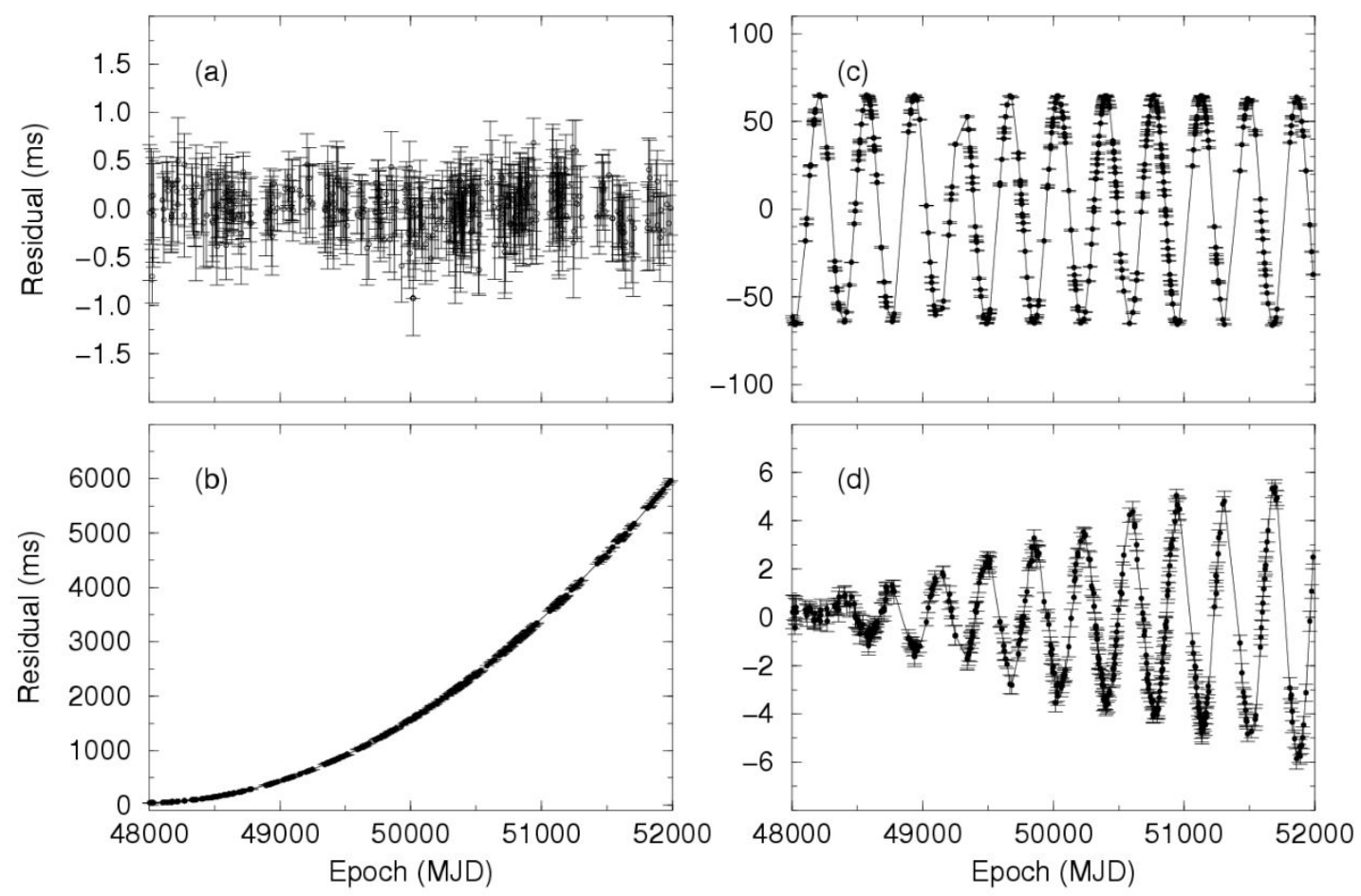

Figure 1.5 Effects on timing residuals for PSR B1133+16 due to errors in the timing model. Plots show (a) a good timing fit, where residuals are not structured and are randomly distributed around zero, (b) an incorrect pulse spin down in the timing model, causing pulses to arrive later and later over time, (c) an error in position which causes the Earth's motion around the Sun to be apparent in the residuals, (d) and an error in proper motion, causes an error in position that grows over time. All parameters but be fit as accurately as possible to achieve high precision in timing. Image taken from Handbook of Pulsar Astronomy (Lorimer \& Kramer, 2012). 
value of $\phi_{o}$ at a time $t_{o}$

$$
\phi(t)=\phi_{o}+f\left(t-t_{o}\right)+\frac{1}{2} \dot{f}\left(t-t_{o}\right)^{2}+\ldots
$$

where $f$ is the pulse frequency. There are corrections for known effects that must be made before $\phi(t)$ can be predicted, making a more complex timing model. For example, effects from other planets in our Solar System, observatory clock corrections, and the difference in light travel time for different points in Earth's orbit all need to be accounted for in timing. Timing models also includes a variety of spin, astrometric, and binary (where applicable) parameters that can be fit for in order to minimize the difference between our model predicted TOAs and measured TOAs. This difference in arrival time is called the residual. These parameters characterize properties both intrinsic to the pulsar as well as extrinsic, like effects on the pulse arrival time due to the ISM. Timing is done using the TEMPO ${ }^{2}$ software package, which applies a least-squares timing model fit. Errors on parameters are determined from the timing parameter covariance matrix after the least-squares fit.

Radio emission detected from pulsars is very weak; single pulses from MSPs are often too faint to detect over the noise. In addition, the pulse shape varies between pulses. As a result, pulses need to be summed in order to construct a stable average pulse profile to be used in the timing. Jitter, intrinsic variations in the pulse shape, causes the underlying pulse to vary from the summed template and introduces noise; an example of these pulse variations can be seen in Figure 1.2.2.

\footnotetext{
${ }^{2}$ http://tempo.sourceforge.net
} 

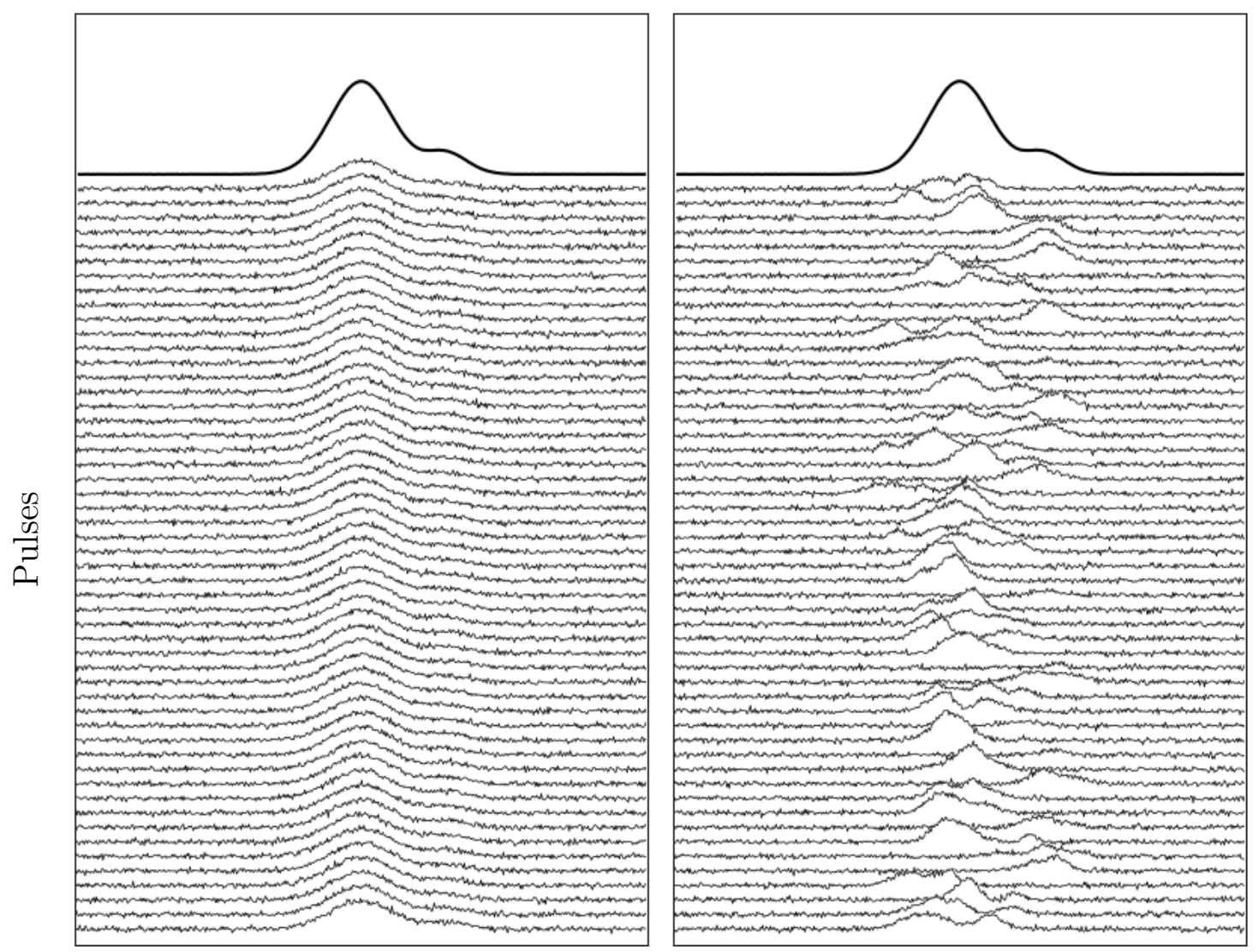

\section{Pulse Phase}

Figure 1.6 Simulated pulse data showing variations in pulse profile that cause jitter, with the summed profile appearing at the top. The simulated data on the left shows pulses that are fairly identical over time, while the pulses on the right show shape variations between pulses (i.e. jitter). Both sets of pulses results in the same averaged pulse profile. 


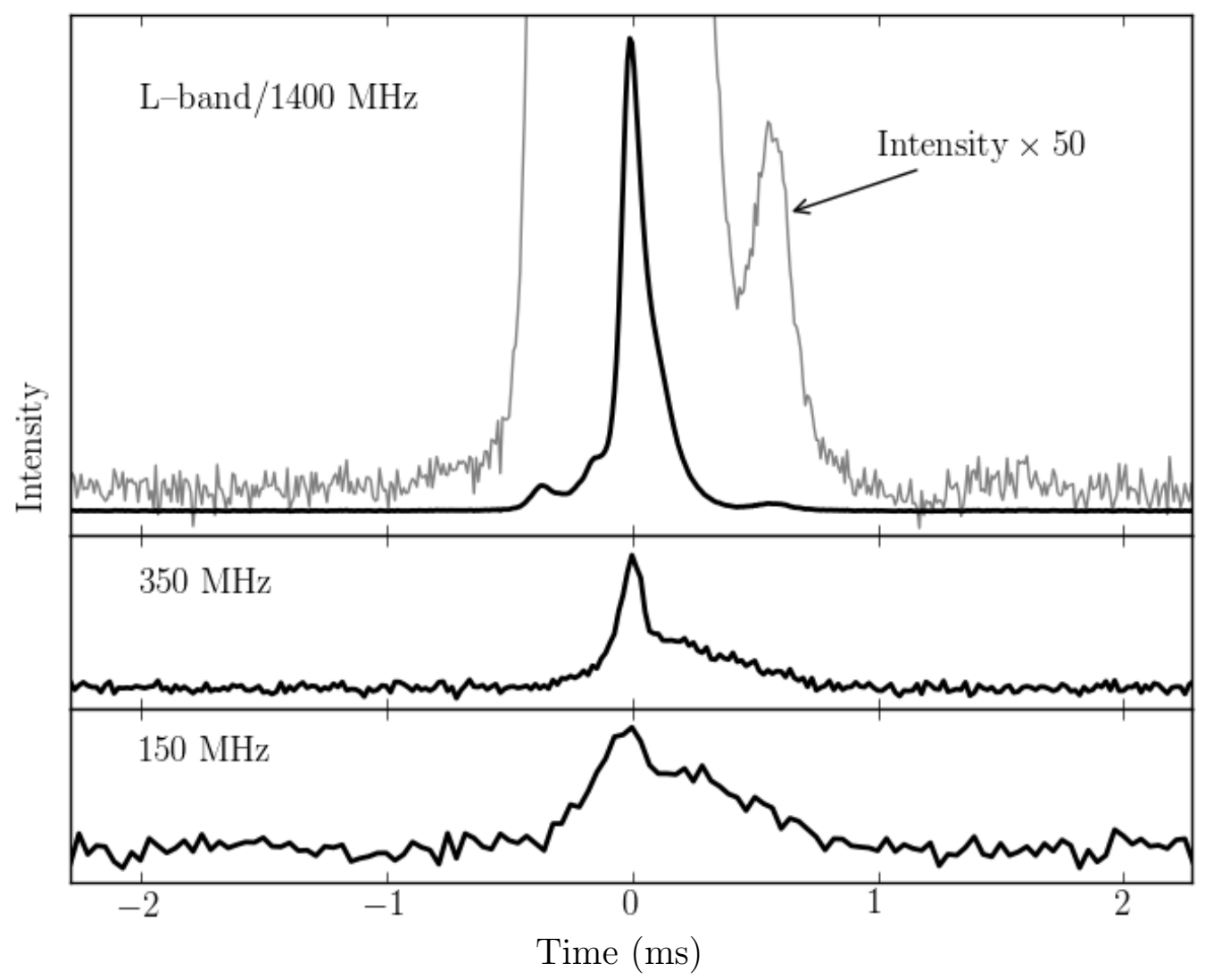

Figure 1.7 Pulse profile shapes for PSR J1713+0747 at different frequencies. The exponential tail visible at 150 and $350 \mathrm{MHz}$ is caused by scattering. Plot from Dolch et al. (2014). 


\subsubsection{Pulsar timing array}

In a PTA, the Earth and a pulsar each make up an end of our GW detector; the effects of a GW on each can be described by the "Earth term" and "pulsar term" respectively. While estimates of the pulsar distance may be known, they are not precise enough to enable detection of the change in distance due a GW perturbation. As a result, the pulsar term will not be correlated between pulsars. The Earth term will show a correlation between MSPs that is related to the angular separation between pulsars. This correlation function was calculated by Hellings \& Downs (1983)

$$
\alpha_{i j}=\frac{\left(1-\cos \gamma_{i j}\right)}{2} \ln \left(\frac{1-\cos \gamma_{i j}}{2}\right)-\frac{1}{6} \frac{\left(1-\cos \gamma_{i j}\right)}{2}+\frac{1}{3}
$$

where $\gamma_{i j}$ is the angle between two pulsars $(i$ and $j)$ in the network. Due to the quadrupolar nature of GWs, pulsars in the same or opposite directions will show a correlated signal, while the residuals for pulsars at $90^{\circ}$ angles from each other will be anti-correlated. The correlation function is illustrated by the Hellings-Downs curve, seen in Figure 1.8.

There are currently three global PTA efforts: the North American Nanohertz Observatory for Gravitational waves (NANOGrav; Arzoumanian et al., 2015), the European Pulsar Timing Array (EPTA; Desvignes et al., 2016), and the Parkes Pulsar Timing Array (PPTA; Manchester et al., 2013). These three experiments make up the International Pulsar Timing Array (IPTA; Manchester \& IPTA, 2013). Other telescopes like MeerKAT and the Giant Metre-Wave Telescope (GMRT) are 


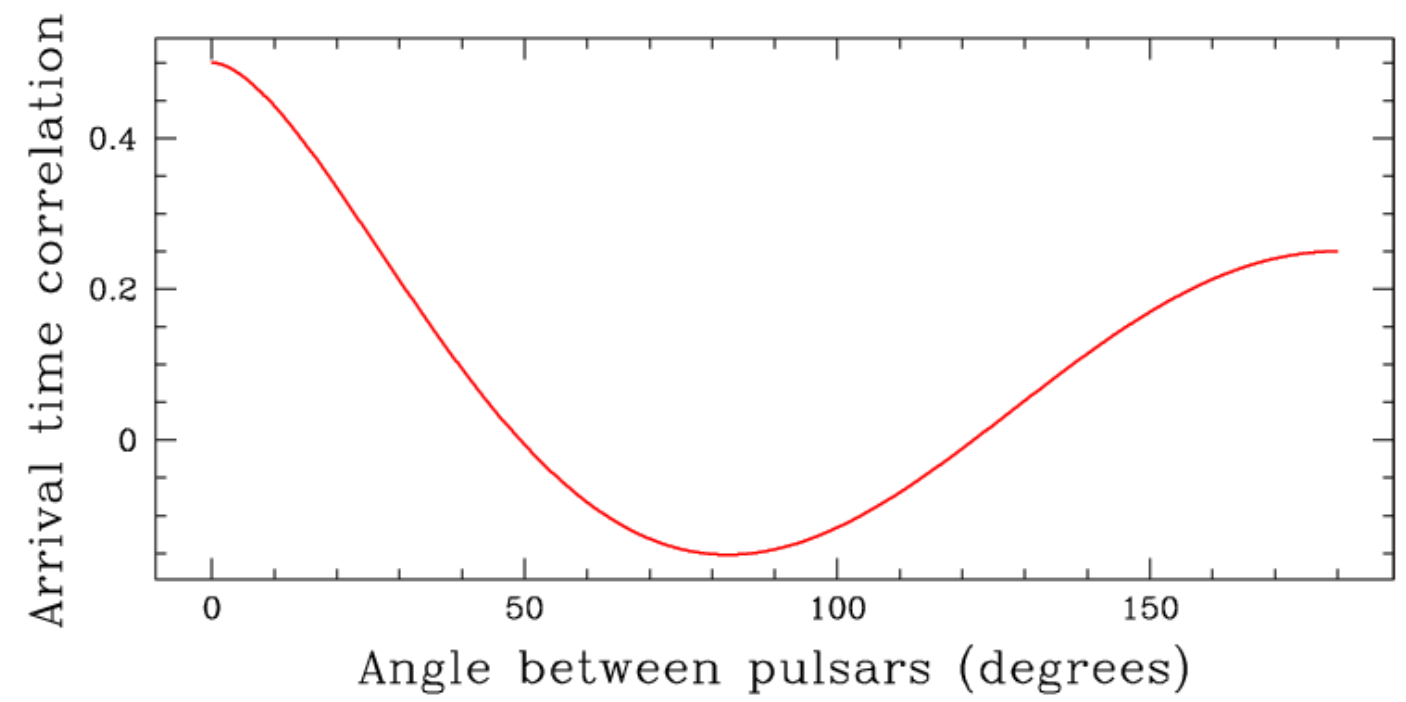

Figure 1.8 The Hellings-Downs curve based on Equation 1.9. Image credit: NANOGrav.

also being used for pulsar timing, and may also be associated with future PTAs incorporated in the IPTA as well. This will be discussed more in Chapter 4.

Timing multiple pulsars forms a network of high precision Galactic clocks; this network is the PTA. The average signal-to-noise of a PTA in the intermediate GW signal limit is

$$
S / N \propto N_{\mathrm{MSP}} T^{1 / 2}\left(\frac{c}{\sigma_{R M S}^{2}}\right)^{3 / 26}
$$

where $N_{\text {MSP }}$ is the number of MSPs in the data set, $T$ is the length of the data span, $\sigma$ is the average rms and $c$ is the average cadence of observations. Sensitivity of a PTA improves as more MSPs are added to the network; it is therefore essential to add as many well-timed MSPs as possible. A perturbation due to a passing GW at the Earth would create a correlated signal across the multi-pulsar network.

Taylor et al. (2016) calculate the probability of a GW detection as a function 
of observation time. Using current estimates of merger rates, the probability of a GW detection by PTAs in the next 6 years is $\sim 80 \%$. PTAs will expand the range of GW astrophysics by offering constraints of the galaxy merger process, including eccentricity and stellar densities. The most recent NANOGrav limit on the GW stochastic background is $h=1.45 \times 10^{-15}$ at a frequency of $\mathrm{f}=1 \mathrm{yr}^{-1}$ (Arzoumanian et al., 2018a).

\subsection{The interstellar medium}

Radio waves propagating through the interstellar medium (ISM) interact with this plasma in space, causing a variety of effects. The ISM is predominantly made up of hydrogen and helium that was created during the Big Bang. There is roughly one free electron for every 10 neutral hydrogen atoms (He et al., 2013).

\subsubsection{Dispersion}

Free electrons cause frequency-dependent dispersion of the pulse, which can be quantified by the dispersion measure (DM). The DM is the integrated column density of free electrons along the line of sight (LOS) to a pulsar

$$
\mathrm{DM}=\int_{0}^{d} n_{e}(l) d l
$$

where $n_{e}$ is the free electron density along a LOS $l$ and $d$ is the pulsar distance. By assuming a model for the Galactic electron density (Cordes \& Lazio, 2002, i.e. NE2001;), DM can also be used to estimate the pulsar distance. 


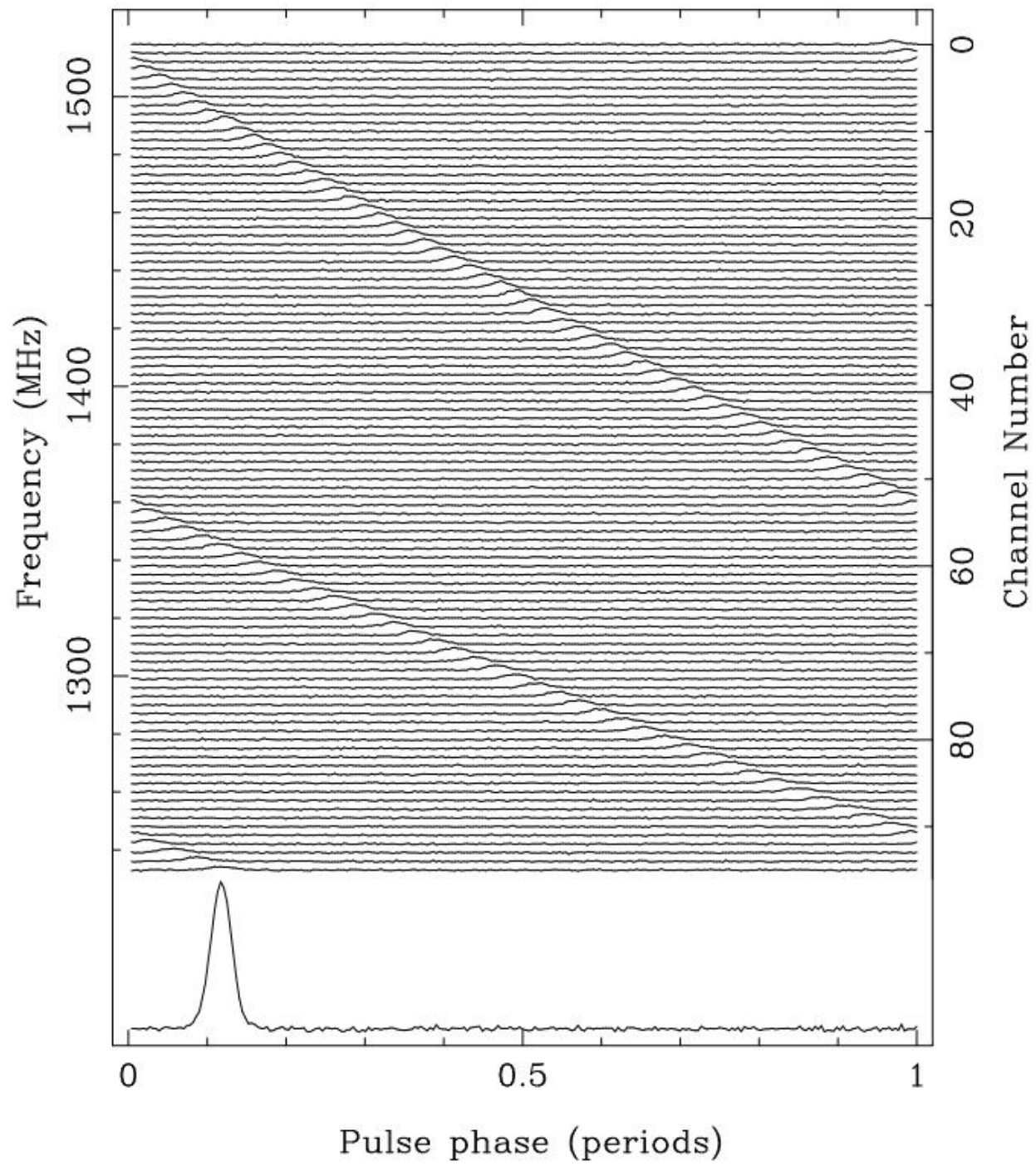

Figure 1.9 Frequency-dependent dispersion due to free electrons in the ISM. Plot taken from Handbook of Pulsar Astronomy (Lorimer \& Kramer, 2012). 
Dispersion causes a frequency-dependent time delay with pulses at lower frequency arriving later than those at higher frequencies. Because of the long distances these pulses are traveling, even a very small $n_{e}$ can result in a measurable change in the pulse arrival time. In order to correct for the time delay caused by DM, timing observations must be done at multiple frequencies to quantify the DM and calculate the difference in arrival times between frequencies

$$
\Delta t \simeq 4.15 \times 10^{6} \mathrm{~ms} \times \mathrm{DM}\left(\frac{1}{\nu_{1}^{2}}-\frac{1}{\nu_{2}^{2}}\right)
$$

where the DM is in pc $\mathrm{cm}^{-3}$ and observing frequencies $\nu_{1}$ and $\nu_{2}$ are in $\mathrm{MHz}$. For example, PSR J1713+0747 has a pulse period of $4.57 \mathrm{~ms}$ and a DM of $15.9 \mathrm{pc} \mathrm{cm}^{-3}$; if observed at $\nu_{1}=820 \mathrm{MHz}$ and $\nu_{2}=2300 \mathrm{MHz}$ (Arzoumanian et al., 2018b, as it is for NANOGrav;), the time delay due to DM would be $86 \mathrm{~ms}$, roughly 19 times larger than the pulse period. The DM varies with time and needs to be fit at each observing epoch. When timing, observations at different frequencies can be timed together to estimate DM for that epoch; any TOAs within that bin are considered to have the same DM. DM is then fit over multiple windows over the data set to characterize any variations and get the clearest picture of the DM for a particular epoch.

For most pulsars, variations in DM exhibit structure over time. DMs can monotonically increase or decrease due to an increasing distance between the Earth and the pulsar, or due to stochastic variations in the ISM. Periodic variations in the form of a smooth sinusoid occur due to the Earth's motion around the Sun, 


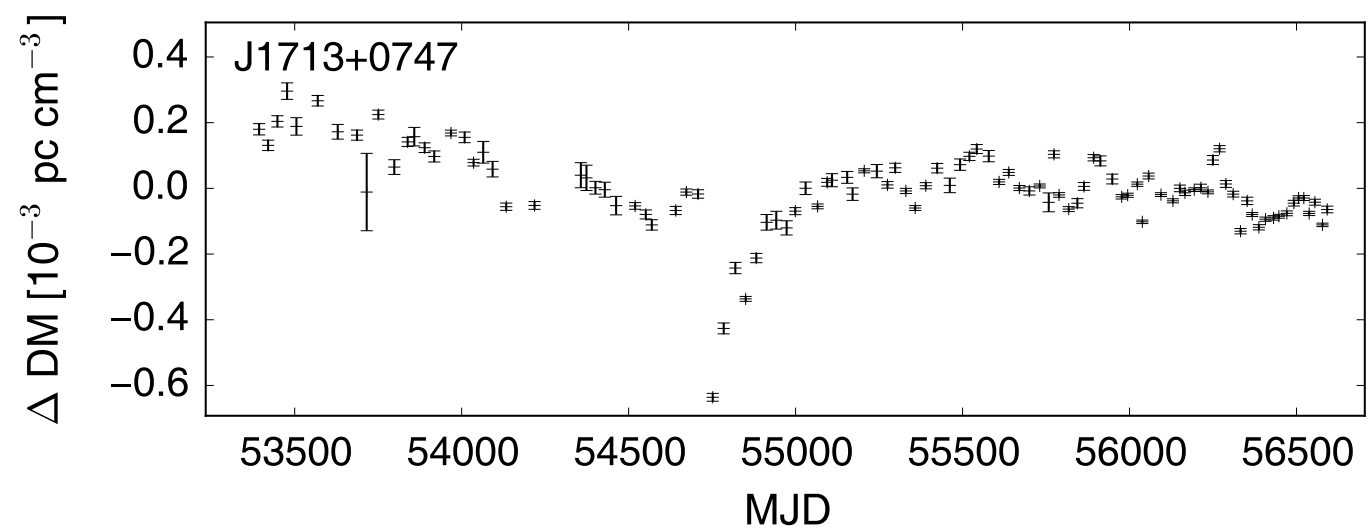

Figure 1.10 DM variations for PSR J1713+0747. Not only does the DM exhibit periodic structure, a significant dip in DM can be seen near the end of 2008 (MJD 54700). DM variations are discussed more throughly in Chapter 2. Data taken from the NANOGrav 9-year data release (Arzoumanian et al., 2015).

or in sharp peaks as the LOS crosses the solar ionosphere. Studying structure can determine whether a DM trend is due to a real astrophysical effect or due to some other noise processes. Variations can occur on timescales of a few hours to years. Accounting for this DM variation timescale is important when determining DM windows for DM correction. Knowledge of such structure is helpful in understanding the ISM. Characterizing the structure seen in these variations along with possible explanations of them are laid out in Chapter 2.

\subsubsection{Scattering}

Pulses are scattered by irregularities in the ISM, causing variable path lengths and therefore varying time delays. This results in a scatter-broadened pulse. As seen with DM, lower frequencies are more affected by the turbulent plasma in the ISM, and so low frequencies experience more scattering than higher frequencies (scatter- 


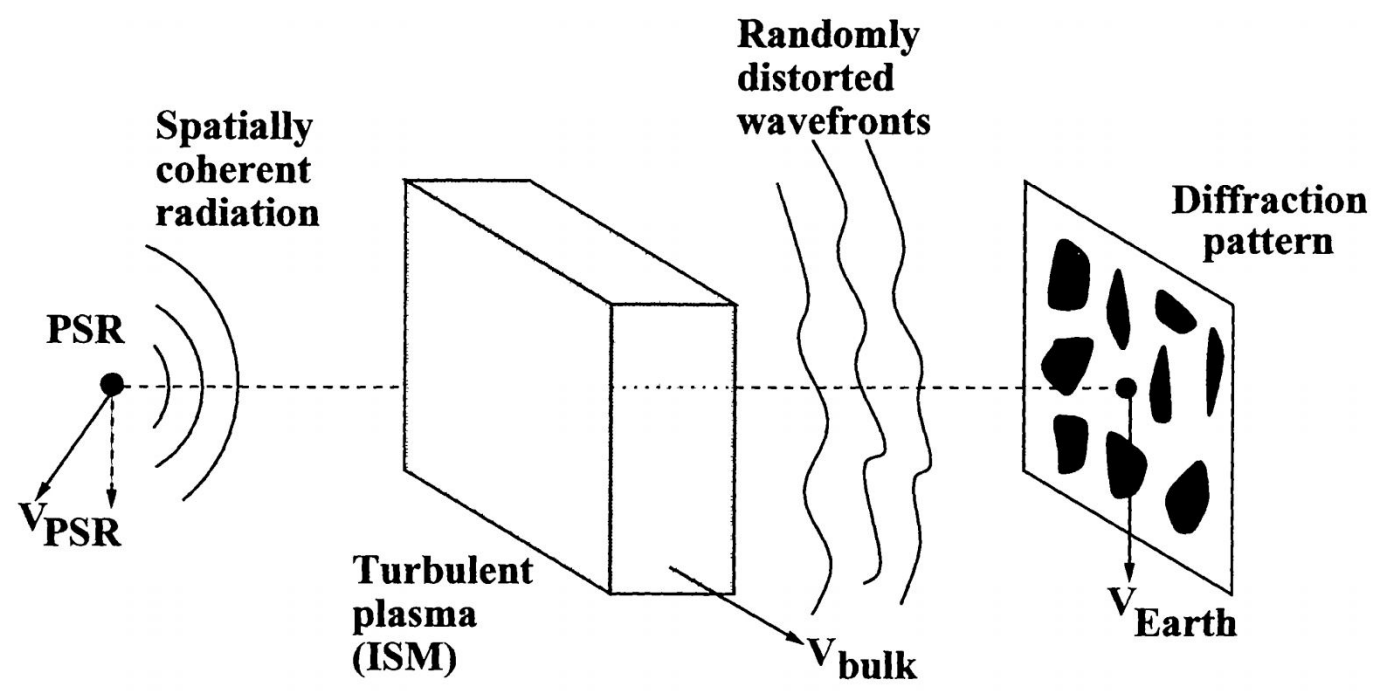

Figure 1.11 Scattering of the pulse by a turbulent plasma screen sitting between the Earth and the pulsar. Image taken from Cordes (2002).

ing scales as $\nu^{-4}$, where $\nu$ is the observing frequency). Because DM and scattering are both frequency dependent, some scattering effects can be absorbed when fitting for DM. Low frequency observations can be used to disentangle scattering contributions from DM. The same irregularities in the ISM that cause scattering also cause scintillation. As the pulse propagation paths change with time, so does the phase of the pulse when it reaches the Earth (i.e. constructive/destructive interference of the pulse). Similar to how stars twinkle when viewed from Earth, the pulse brightness will vary with the modulation of the pulse phase. The effects of scattering in pulsar timing are discussed more in Chapter 4.

\subsection{Using multiple radio telescopes for GW astrophysics}

There are many radio telescopes currently being used for GW detection by PTAs as well as other PTA science. Observing with multiple telescopes improves 
sky coverage and cadence as multiple telescopes can observe the same source.

The EPTA utilizes the Effelsberg 100-m Radio Telescope in Germany, the Lovell Telescope at Jodrell Bank in the UK, the Nançay decimetric radio telescope in France, and the Westerbork Synthesis Radio Telescope in the Netherlands. When the Sardinia Radio Telescope in Italy comes fully online, it will also be used in EPTA science (Desvignes et al., 2016). This group of telescopes has the advantage of providing wide frequency coverage, high cadence, and a long timing baseline for many pulsars.

The PPTA uses the Parkes Observatory in Australia. Parkes is equipped with a 64-m dish and also has a long timing baseline for many pulsars. Parkes is able to observe MSPs in the Southern Hemisphere that are not visible to the other PTAs; this coverage is useful for the IPTA.

NANOGrav observes with the Arecibo Observatory in Puerto Rico and the Green Bank Observatory in West Virginia. The Arecibo Observatory hosts a 305-m dish; due to this large collecting area, Arecibo is extremely sensitive. Because of this size, the dish cannot move and therefore has limited range on the sky that it can observe. The Green Bank Observatory on the other hand can see $~ 85 \%$ of the sky; at $100-\mathrm{m}$, it is the largest steerable object on land. Both Arecibo and Green Bank are capable of observing large bandwidths, providing the highest precision TOAs. 


\subsubsection{Telescopes starting to do PTA science}

The Giant Metrewave Radio Telescope (GMRT) and Ooty Radio Telescope (ORT) are both part of the National Centre for Radio Astrophysics (NCRA) in India. The ORT is a 530-m long and 30-m cylindrical parabolic antenna. The GMRT is made up of 30 45-m dishes and offers frequency coverage from 150 to 1500 MHz. This lower coverage will make the GMRT useful for noise and ISM studies. The Very Large Array (VLA) in New Mexico is comprised of 27 25-m dishes. Pulsar timing has been done at the VLA for the past few years; the VLA can observe PSR J0437-4715 in the south, which is a particularly bright MSP with high timing precision. The array can also observe at high frequencies (2 to $4 \mathrm{GHz}$ ) which is useful for ISM mitigation. The MeerKAT radio telescope is currently operating and will be made up of 64 dishes that are each comprised of a 13.5-m reflector and 3.8-m subreflector. MeerKAT will make very sensitive TOA measurements, and will be capable of forming sub-arrays to observe multiple pulsars at once.

The Canadian Hydrogen Intensity Mapping Experiment (CHIME) telescope and Five-hundred-meter Aperture Spherical radio Telescope (FAST) are both in the commissioning phase and will be used to time pulsars once completed. CHIME consists of 4 100-m by 20-m semi-cylinders in British Columbia. The telescope cannot be steered, it observes the sky overhead as the Earth turns. CHIME can observe up to 10 pulsars simultaneously (observation duration depends on declination, MSPs may be visible for $\sim 10$ minutes or less; $\mathrm{Ng}, 2017$ ). CHIME will observe frequencies from 400 to $800 \mathrm{MHz}$. Daily observations with CHIME will be very useful for DM 
correction and characterization. FAST in China is very similar to the Arecibo Observatory, only much bigger (500-m) and can observe a larger range in declination. FAST will achieve incredibly sensitive observations of MSPs (Hobbs et al., 2014).

\subsubsection{Combining timing data}

There can be many difficulties in combining data from multiple telescopes as each telescope has its own aspects that may need to be taken into account. Telescope backends are also individual to the telescope and are replaced/upgraded over time, which needs to be accounted for when incorporating older data. Different observatories may use different file formats, header information, and hardware/software for data acquisition. Data processing is done differently in each PTA, and timing models can vary between PTAs. For example, DM is corrected for by a different method, different timing parameters (e.g. using a solar wind model) may be used, among other others.

\subsection{Astrophysics across a wide frequency range}

In this thesis multiple radio observations at various frequencies between 322 $\mathrm{MHz}$ and $10 \mathrm{GHz}$ are used to conduct astrophysical research related to GW detection using PTAs. In Chapter 2, we discuss DM variations seen in the NANOGrav 9-year data release using wideband multi-frequency observations. Specifically, we measure and analyze trends seen in the DM time series, propose sources of these trends, and identify timescales over which the DM varies beyond measurement er- 
rors and therefore can no longer be considered constant. In Chapter 3, we analyze a previously published work that identifies the galaxy NGC 3115 as being an AGN potentially situated outside of the galactic photocenter. We investigate the possibilities that the source is a SMBH binary or a post-merger recoiling SMBH. In Chapter 4, we compare DM measurements obtained with dual-frequency GMRT observations to those calculated in the NANOGrav 11-year data release and assess the relative precision of the GMRT DM measurements. We also examine data taken with the GMRT to identify its potential for inclusion in the IPTA. In Chapter 5, conclusions are put forth based on the works presented here and discuss avenues for future works. 


\section{Chapter 2}

\section{The NANOGrav Nine-Year Data Set:}

\section{Measurement and Analysis of Variations in Dispersion Measures}

\subsection{Abstract}

We analyze dispersion measure $(\mathrm{DM})$ variations of 37 millisecond pulsars in the nine-year North American Nanohertz Observatory for Gravitational Waves (NANOGrav) data release and constrain the sources of these variations. DM variations can result from a changing distance between Earth and the pulsar, inhomogeneities in the interstellar medium, and solar effects. Variations are signicant for nearly all pulsars, with characteristic timescales comparable to or even shorter than the average spacing between observations. Five pulsars have periodic annual variations, 14 pulsars have monotonically increasing or decreasing trends, and 14 pulsars show both effects. Of the four pulsars with linear trends that have line-of-sight velocity measurements, three are consistent with a changing distance and require an overdensity of free electrons local to the pulsar. Several pulsars show correlations

Published as M. L . Jones et al. 2017, ApJ, 841, 125.

Contributing authors: M. A. McLaughlin, M. T. Lam, J. M. Cordes, L. Levin, S. Chatterjee, Z. Arzoumanian, K. Crowter, P. B. Demorest, T. Dolch, J. A Ellis, R. D. Ferdman, E. Fonseca, M. E. Gonzalez, G. Jones, T. J. W. Lazio, D. J. Nice, T. T. Pennucci, S. M. Ransom, D. R. Stinebring, I. H. Stairs, K. Stovall, J. K. Swiggum, W. W. Zhu 
between DM excesses and lines of sight that pass close to the Sun. Mapping of the DM variations as a function of the pulsar trajectory can identify localized interstellar medium features and, in one case, an upper limit to the size of the dispersing region of 4 AU. Four pulsars show roughly Kolmogorov structure functions(SFs), and another four show SFs less steep than Kolmogorov. One pulsar has too large an uncertainty to allow comparisons. We discuss explanations for apparent departures from a Kolmogorov-like spectrum, and we show that the presence of other trends and localized features or gradients in the interstellar medium is the most likely cause.

\subsection{Introduction}

The principal goal of the North American Nanohertz Observatory for Gravitational Waves (NANOGrav; McLaughlin, 2013) is to detect gravitational waves in the nanohertz regime of the gravitational wave spectrum using a pulsar timing array (PTA). Sensitivity improves as more millisecond pulsars (MSPs) are added to the PTA, and therefore it is essential to have as many well-timed MSPs as possible (Siemens et al., 2013; Vigeland \& Siemens, 2016). For every MSP, we must construct an accurate timing model that accounts for all known effects on the pulsar times-of-arrival (TOAs) over decade timescales (Jenet et al., 2005; Cordes \& Shannon, 2010). One of the parameters that must be fit in the timing model is the dispersion measure (DM; Lorimer \& Kramer, 2012). As the pulsar signal travels through the interstellar medium (ISM), it encounters ionized plasma and electron 
density variations along the way. The DM is the integrated column density of free electrons along the line of sight to a pulsar

$$
\mathrm{DM}=\int_{0}^{d} n_{e}(l) d l
$$

where $n_{e}$ is the free electron density along a line of sight $l$ and $d$ is the pulsar distance. When the pulsar signal propagates through the ISM, interactions with these free electrons cause dispersion that is characterized by a frequency dependent time delay

$$
\Delta t \simeq 4.15 \times 10^{6} \mathrm{~ms} \times \mathrm{DM}\left(\frac{1}{\nu_{1}^{2}}-\frac{1}{\nu_{2}^{2}}\right)
$$

where $\nu_{1}$ and $\nu_{2}$ are two different frequencies in $\mathrm{MHz}$ and $\mathrm{DM}$ is in $\mathrm{pc} \mathrm{cm}^{-3}$. Observing at least two frequencies is necessary to solve for the DM for a measured time delay. This time delay can be significant when compared to the pulsar period, and therefore the DM must be fit when creating a timing model and corrected for at each epoch (e.g. Demorest et al., 2013; Arzoumanian et al., 2015).

Inhomogeneities in the ISM, solar wind, and differences in the relative velocity of the pulsar and the Earth can change the free electron density along the line of sight (LOS; Lam et al., 2016). The result is a DM that varies with time, changing on timescales of hours to years. In this paper we discuss the variations seen in the NANOGrav 9-year data release (Arzoumanian et al., 2015), constrain the possible sources of these variations, and use these constraints to characterize the ISM along the LOS. 
In $\S 2$, we discuss the data used for this analysis. In $\S 3$, we discuss the significance and trends seen in the DM time series. In $\S 4$, we perform a structure function (SF) analysis on select MSPs and put the results in the context of a Kolmogorov spectrum. In $\S 5$, we discuss the results of these analyses and in $\S 6$ we present conclusions.

\subsection{The NANOGrav 9-year data set}

Our analysis uses data from the NANOGrav 9-year data set (Arzoumanian et al., 2015). Pulsars were included in the data set based on the anticipated stability of their timing, their TOA precision, and their detection over a wide frequency range. Of the 37 MSPs included in the data release, 17 were reported on in Demorest et al. (2013). Observations took place roughly once a month between 2004 and 2013 with observing time spans of individual pulsars ranging from 0.6 to 9.0 years. Those MSPs with declinations between 0 and $39^{\circ}$ were observed with the $305-\mathrm{m}$ William E. Gordon Telescope at the Arecibo Observatory, and the rest were observed with the 100-m Robert C. Byrd Green Bank Telescope (GBT) of the National Radio Astronomy Observatory (NRAO); PSRs J1713+0747 and B1937+21 were observed with both telescopes. Every MSP was observed at multiple frequencies to account for frequency-dependent dispersion effects. Dual frequency observations occurred within $\sim 1$ hour at Arecibo and within several days at the GBT. The typical length of an observation was $\sim 25$ minutes. A more detailed and thorough description of these observations can be found in Arzoumanian et al. (2015). 
For each pulsar, the DM was measured at nearly every observing epoch and recorded using the DMX parameter as part of the TEMPO software package ${ }^{1}$, where DMX models DM as constant over a chosen time window (14 days in this case). The $\Delta \mathrm{DM}$, an offset from a globally fixed fiducial DM, is fit as a free parameter in the timing model. The possible errors in $\mathrm{DM}(t)$ estimation using this method are discussed in Lam et al. (2015). Errors on DM are $1 \sigma$ and are determined from the timing-parameter covariance matrix after the least-squares timing model fit. Data from early single-receiver observations were omitted for PSRs J1741+1351, $\mathrm{J} 1853+1303$, J1910+1256, J1944+0907, and B1953+29 as it was not possible to independently measure DM and other timing properties. We plot DMs vs time (i.e., $\operatorname{DM}(t))$ for all of the pulsars in Figures 2.1 through 2.5. Values from the 9-year data release used in this analysis can be seen in Table 2.1. Partial $\operatorname{DM}(t)$ data spans have already been published for 15 pulsars (PSRs J0340+4130, J0613-0200, J16142230, J1713+0747, J1738+0333, J1741+1351, J1744-1134, B1855+09, J1909-3744, J1910+1256, J1918-0642, B1937+21, J1944+0907, J2010-1323, and J2302+4442) in Levin et al. (2016).

\subsection{Determining significance and trends in the variations}

Variability in the DM timeseries can be seen for many pulsars; it has been a long known effect. The first detection of temporal variations was for the Crab pulsar (Rankin \& Roberts, 1971). These variations were later determined to be most likely due to variations in the surrounding nebula (Isaacman \& Rankin, 1977). The Vela

\footnotetext{
${ }^{1}$ TEMPO software package: http://tempo.sourceforge.net
} 
pulsar exhibits a decreasing time-dependent DM attributed to the pulsar motion through the enveloping supernova remnant (Hamilton et al., 1985).

We first determine whether the DM variations we see are significant or if they are consistent (within errors) with a constant DM value. We calculate the reduced $\chi^{2}$ for each pulsar as

$$
\chi_{r}^{2}=\frac{1}{N_{\mathrm{DOF}}} \sum \frac{(\mathrm{DM}(t)-\overline{\mathrm{DM}})^{2}}{\sigma(t)^{2}}
$$

where $N_{\text {DOF }}$ is the number of degrees of freedom, $\overline{\mathrm{DM}}$ is the average DM value for the data span for a pulsar (in this model), and $\sigma(t)$ is the error associated with each DM(t) value. All but two of the pulsars (PSRs J1923+2515 and J2214+3000) have $\chi_{r}^{2} \geq 1$. Of these, we identify 15 pulsars as showing moderate variations (those with $\left.1 \leq \chi_{r}^{2} \leq 10\right)$, and 20 pulsars with significant variations $\left(\chi_{r}^{2} \geq 10\right)$. We therefore conclude that the DMs are intrinsically variable for all of the MSPs in our sample with the possible exceptions of J1923+2515 and J2214+3000, which both show visible variation at a low level despite the statistical test. Both of these pulsars have short data sets (2.2 and 2.1 years, respectively).

\subsubsection{Systematic variations}

DMs can vary in many ways, with components that appear linear, periodic, or random. Here we consider systematic DM variations such as linear trends and periodicities. Stochastic contributions are discussed in Section 2.5. Sources of linear trends and periodicities include a changing distance between the Earth and the 
Table 2.1 Properties of NANOGrav MSPs in the 9-Year Data Release.

\begin{tabular}{|c|c|c|c|c|c|c|c|c|c|}
\hline PSR & $\begin{array}{r}\mu_{\lambda} \\
\left(\operatorname{mas~yr}^{-1}\right)\end{array}$ & $\begin{array}{r}\mu_{\beta} \\
\left(\operatorname{mas~yr}^{-1}\right)\end{array}$ & $\begin{array}{r}\mu_{\alpha} \\
\left(\operatorname{mas~yr}^{-1}\right)\end{array}$ & $\begin{array}{r}\mu_{\delta} \\
\left(\operatorname{mas~yr}^{-1}\right)\end{array}$ & $\begin{array}{r}\mathrm{DM} \\
\left(\mathrm{pc} \mathrm{cm}^{-3}\right)\end{array}$ & $\begin{array}{l}\mathrm{d}_{\mathrm{DM}} \\
(\mathrm{kpc})\end{array}$ & $\begin{array}{l}\mathrm{d}_{\mathrm{PX}} \\
(\mathrm{kpc})\end{array}$ & $\chi_{r}^{2}$ & $\begin{array}{l}v_{T} \\
\left(\mathrm{~km} \mathrm{~s}^{-1}\right)\end{array}$ \\
\hline $\mathrm{J} 0023+0923$ & $-13.9(2)$ & $-1(1)$ & $-12.3(6)$ & $-6.7(9)$ & 14.3 & $0.7(2)$ & - & 4.2 & $46(13)$ \\
\hline $\mathrm{J} 0030+0451$ & $-5.52(1)$ & $3.0(5)$ & $-6.3(2)$ & $0.6(5)$ & 4.3 & $0.3(1)$ & $0.30(2)$ & 11 & $8.9(7)$ \\
\hline $\mathrm{J} 0340+4130$ & $-2.4(8)$ & $-4(1)$ & $-1.3(7)$ & $-5(1)$ & 49.6 & $1.7(4)$ & - & 6.8 & $38(12)$ \\
\hline J0613-0200 & $2.12(2)$ & $-10.34(4)$ & $1.85(2)$ & $-10.39(4)$ & 38.8 & $1.7(4)$ & $1.1(2)$ & 70 & $55(10)$ \\
\hline J0645+5158 & $2.1(1)$ & $-7.3(2)$ & 1.4(1) & $-7.5(2)$ & 18.2 & $0.7(2)$ & $0.8(3)$ & 2.7 & $29(11)$ \\
\hline J0931-1902 & - & - & - & - & 41.5 & $1.8(5)$ & - & 2.2 & - \\
\hline $\mathrm{J} 1012+5307$ & $13.9(1)$ & $-21.7(3)$ & $2.5(2)$ & $-25.6(2)$ & 9.0 & $0.4(1)$ & - & 1.8 & $49(12)$ \\
\hline J1024-0719 & $-14.36(6)$ & $-57.8(3)$ & $-35.2(1)$ & $-48.0(2)$ & 6.5 & $0.4(1)$ & - & 15 & $113(28)$ \\
\hline J1455-3330 & $8.16(7)$ & $0.5(3)$ & $7.9(1)$ & $-2.0(3)$ & 13.6 & $0.5(1)$ & - & 2.4 & $19(4)$ \\
\hline J1600-3053 & $0.47(2)$ & $-7.0(1)$ & $-0.95(3)$ & $-7.0(1)$ & 52.3 & $1.6(4)$ & $3.0(8)$ & 42 & $100(27)$ \\
\hline J1614-2230 & $9.46(2)$ & $-31(1)$ & $3.8(2)$ & $-32(1)$ & 34.5 & $1.3(3)$ & $0.65(5)$ & 20 & $100(8)$ \\
\hline $\mathrm{J} 1640+2224$ & $4.20(1)$ & $-10.73(2)$ & $2.09(1)$ & $-11.33(2)$ & 18.5 & $1.2(3)$ & - & 295 & $66(16)$ \\
\hline J1643-1224 & $5.56(8)$ & $5.3(5)$ & $6.2(1)$ & $4.5(5)$ & 62.4 & $2.3(6)$ & - & 112 & $84(22)$ \\
\hline $\mathrm{J} 1713+0747$ & $5.260(2)$ & $-3.442(5)$ & $4.918(2)$ & $-3.914(5)$ & 16.0 & $0.9(2)$ & $1.18(4)$ & 29 & $35(1)$ \\
\hline $\mathrm{J} 1738+0333$ & $6.6(2)$ & $6.0(4)$ & $6.9(2)$ & $5.8(4)$ & 33.8 & $1.4(4)$ & - & 5.0 & $59(17)$ \\
\hline $\mathrm{J} 1741+1351$ & $-8.8(1)$ & $-7.6(2)$ & $-9.1(1)$ & $-7.2(2)$ & 24.2 & $0.9(2)$ & - & 4.7 & $50(11)$ \\
\hline J1744-1134 & $19.01(2)$ & $-8.68(8)$ & $18.76(2)$ & $-9.20(8)$ & 3.1 & $0.4(1)$ & $0.41(2)$ & 17 & $41(2)$ \\
\hline J1747-4036 & $0.1(8)$ & $-6(1)$ & $0(1)$ & $-6(1)$ & 153.0 & $3.3(8)$ & - & 133 & - \\
\hline J1832-0836 & - & - & - & - & 28.2 & $1.1(3)$ & - & 16 & - \\
\hline $\mathrm{J} 1853+1303$ & $-1.8(2)$ & $-2.9(4)$ & $-1.48(2)$ & $-3.1(4)$ & 30.6 & $2.0(5)$ & - & 5.8 & $32(9)$ \\
\hline B1855+09 & $-3.27(1)$ & $-5.10(3)$ & $-2.651(15)$ & $-5.45(3)$ & 13.3 & $1.2(3)$ & 一 & 1335 & $34(9)$ \\
\hline $\mathrm{J} 1903+0327$ & $-3.5(3)$ & $-6.2(9)$ & $-2.7(3)$ & $-6.5(9)$ & 297.6 & $6(2)$ & - & 27 & $202(71)$ \\
\hline J1909-3744 & $-13.868(4)$ & $-34.34(2)$ & $-9.518(4)$ & $-35.79(2)$ & 10.4 & $0.5(1)$ & $1.07(4)$ & 1375 & $188(7)$ \\
\hline $\mathrm{J} 1910+1256$ & $-0.7(1)$ & $-7.2(2)$ & $0.3(1)$ & $-7.2(2)$ & 38.1 & $2.3(6)$ & - & 2.7 & $79(21)$ \\
\hline J1918-0642 & $-7.93(2)$ & $-4.85(9)$ & $-7.18(3)$ & $-5.90(9)$ & 26.6 & $1.2(3)$ & $0.9(2)$ & 171 & $40(9)$ \\
\hline $\mathrm{J} 1923+2515$ & $-9.5(2)$ & $-12.8(5)$ & $-6.6(2)$ & $-14.5(5)$ & 18.9 & $1.6(4)$ & - & 0.9 & $121(30)$ \\
\hline B1937+21 & $-0.02(1)$ & $-0.41(2)$ & $0.07(1)$ & $-0.40(2)$ & 71.0 & $3.6(7)$ & - & 1162 & $7(1)$ \\
\hline $\mathrm{J} 1944+0907$ & $9.4(1)$ & $-25.5(4)$ & $14.37(11)$ & $-23.1(4)$ & 24.3 & $1.8(5)$ & - & 147 & $232(64)$ \\
\hline $\mathrm{J} 1949+3106$ & $13(15)$ & 10(13) & $10(11)$ & 13(16) & 164.1 & $3.6(9)$ & - & 1.4 & - \\
\hline B1953+29 & $-1.8(9)$ & $-4.4(14)$ & $-0.4(12)$ & $-5(1)$ & 104.5 & $5(1)$ & - & 6.6 & $113(24)$ \\
\hline J2010-1323 & $1.16(4)$ & $-7.3(4)$ & $2.71(9)$ & $-6.9(4)$ & 22.2 & $1.0(3)$ & - & 70 & $35(11)$ \\
\hline $\mathrm{J} 2017+0603$ & 2.3(6) & $-0.1(7)$ & $2.2(7)$ & $0.5(6)$ & 23.9 & $1.6(4)$ & - & 2.8 & - \\
\hline $\mathrm{J} 2043+1711$ & $-8.97(7)$ & $-8.5(1)$ & $-5.85(7)$ & $-10.9(1)$ & 20.7 & $1.7(4)$ & $1.3(4)$ & 6.3 & $100(23)$ \\
\hline $\mathrm{J} 2145-0750$ & $-12.04(4)$ & $-3.7(4)$ & $-10.1(1)$ & $-7.5(4)$ & 9.0 & $0.6(2)$ & $0.8(2)$ & 24 & $48(12)$ \\
\hline $\mathrm{J} 2214+3000$ & $17.1(5)$ & $-10.5(9)$ & $20.0(6)$ & $-1.7(8)$ & 22.6 & $1.5(4)$ & - & 1.0 & $143(38)$ \\
\hline $\mathrm{J} 2302+4442$ & $-3.3(6)$ & $-1(2)$ & $-2(1)$ & $-3(2)$ & 13.7 & $1.1(3)$ & - & 1.5 & - \\
\hline $\mathrm{J} 2317+1439$ & $0.19(2)$ & $3.80(7)$ & $-1.39(3)$ & $3.55(6)$ & 21.9 & $0.8(2)$ & $1.3(4)$ & 18732 & $14(4)$ \\
\hline
\end{tabular}

Notes. Columns are pulsar name, ecliptic proper motion (longitude and latitude), proper motion in RA and Dec, the DM, the DM-derived distance, the parallax-derived distance, the reduced chi-squared of the DM time series prior to any fitting, and the transverse velocity. The ecliptic proper motions and DMs were calculated for the 9-year data release (Arzoumanian et al., 2015). Proper motion in RA and Dec as well as parallax distances were calculated through timing observations and discussed in Matthews et al. (2016). The DM derived distances were calculated from the NE2001 model assuming 20\% error (Cordes \& Lazio 2002). The value for $\chi_{r}^{2}$ was calculated using Equation 2.3. Dashes indicate that no significant measurement was possible. The transverse velocity $v_{T}$ was calculated from the proper motion and the distance estimate with the smaller error (i.e. $d_{D M}$ or $\left.d_{P X}\right)$. 
Table 2.2 Fitted Trends in the DM Time Series for MSPs in the 9-Year Release

\begin{tabular}{|c|c|c|c|c|c|c|c|c|c|}
\hline PSR & Trend & $\begin{array}{l}d \mathrm{DM} / d t \\
\left(10^{-3} \mathrm{pc} \mathrm{cm}^{-3} \mathrm{yr}^{-1}\right)\end{array}$ & $\begin{array}{l}\text { Amplitude } \\
\left(10^{-4} \mathrm{pc} \mathrm{cm}^{-3}\right)\end{array}$ & $\begin{array}{l}\text { Period } \\
\text { (days) }\end{array}$ & $\chi_{r}^{2}$ & $\begin{array}{l}\mathrm{P}_{\mathrm{LS}} \\
\text { (days) }\end{array}$ & $\begin{array}{l}\text { FAP } \\
(\%)\end{array}$ & $\begin{array}{l}\text { Length } \\
\text { (days) }\end{array}$ & $\begin{array}{r}\delta t \\
\text { (days) }\end{array}$ \\
\hline J0023+0923 & None & - & - & - & - & - & - & 841 & - \\
\hline $\mathrm{J} 0030+0451$ & Periodic & - & $1.2(3)$ & $373(5)$ & 9.2 & 371 & 6.3 & 3204 & 33 \\
\hline $\mathrm{J} 0340+4130$ & Linear & $0.88(9)$ & - & - & 1.7 & 241 & 6.3 & 613 & 73 \\
\hline J0645+5158 & Periodic & - & $0.9(3)$ & $377(29)$ & 2.6 & 199 & 0.54 & 881 & 78 \\
\hline J0931-1902 & None & - & - & - & - & - & - & 235 & - \\
\hline $\mathrm{J} 1012+5307$ & Linear & $0.11(2)$ & - & - & 0.94 & - & - & 3368 & 2286 \\
\hline J1024-0719 & Linear & $0.39(2)$ & - & - & 2.7 & - & - & 1467 & 148 \\
\hline J1455-3330 & Linear & $0.15(2)$ & - & - & 1.0 & 361 & 7.3 & 3368 & 904 \\
\hline J1614-2230 & Periodic & - & $3.1(5)$ & $370(9)$ & 11 & 370 & 0.17 & 1860 & 14 \\
\hline J1643-1224 & Both & $-1.02(3)$ & $8(1)$ & $387(4)$ & 10 & 387 & 0.01 & 3293 & 104 \\
\hline $\mathrm{J} 1738+0333$ & Linear & $-0.8(2)$ & - & - & 1.4 & - & - & 1456 & 213 \\
\hline $\mathrm{J} 1741+1351$ & Linear & $-0.12(4)$ & - & - & 1.9 & - & - & 1224 & 287 \\
\hline J1744-1134 & Both & $-0.069(7)$ & $0.4(2)$ & $383(16)$ & 8.0 & - & - & 3369 & 66 \\
\hline J1747-4036 & Linear & $-7.3(4)$ & - & - & 10.0 & 459 & 6.7 & 608 & 16 \\
\hline J1832-0836 & None & - & - & - & - & - & - & 231 & - \\
\hline $\mathrm{J} 1853+1303$ & Linear & $0.12(9)$ & - & - & 5.2 & - & - & 1468 & 361 \\
\hline B1855+09 & Both & $0.382(7)$ & $0.5(3)$ & $364(11)$ & 15.7 & - & - & 3240 & 27 \\
\hline $\mathrm{J} 1903+0327$ & Both & $-3.0(4)$ & $31(6)$ & $375(11)$ & 12 & 371 & 1.0 & 1456 & 99 \\
\hline J1909-3744 & Both & $-0.239(4)$ & $0.7(1)$ & $366(5)$ & 28 & 366 & 0.25 & 3306 & 9 \\
\hline $\mathrm{J} 1910+1256$ & Linear & $0.51(6)$ & - & - & 0.90 & 404 & 2.9 & 2574 & 443 \\
\hline $\mathrm{J} 1923+2515$ & None & - & - & - & - & - & - & 803 & - \\
\hline J1944+0907 & Linear & $1.3(2)$ & - & - & 44 & - & - & 1467 & 43 \\
\hline $\mathrm{J} 1949+3106$ & Periodic & - & $10(3)$ & $391(37)$ & 1.0 & - & - & 455 & 51 \\
\hline B1953+29 & Both & $-1.3(3)$ & $3(2)$ & $356(72)$ & 2.1 & - & - & 1967 & 136 \\
\hline J2010-1323 & Both & $0.38(2)$ & $2.2(4)$ & $372(9)$ & 14 & 372 & 0.56 & 1490 & 16 \\
\hline $\mathrm{J} 2017+0603$ & Both & $0.23(7)$ & $2.3(5)$ & $440(37)$ & 0.94 & - & - & 609 & 38 \\
\hline $\mathrm{J} 2043+1711$ & Both & $-0.12(4)$ & $1.0(4)$ & $390(38)$ & 3.7 & - & - & 834 & 189 \\
\hline $\mathrm{J} 2145-0750$ & Linear & $0.08(2)$ & - & - & 18 & - & - & 3318 & 568 \\
\hline $\mathrm{J} 2214+3000$ & Periodic & - & $4(1)$ & $319(25)$ & 0.83 & - & - & 755 & 17 \\
\hline $\mathrm{J} 2302+4442$ & Linear & $-0.6(2)$ & - & - & 1.5 & - & - & 613 & 202 \\
\hline $\mathrm{J} 2317+1439$ & Both & $-0.550(9)$ & $0.9(3)$ & $311(6)$ & 321 & - & - & 3243 & 5 \\
\hline PSR & Trend & $\begin{array}{l}d \mathrm{DM} / d t \\
\left(10^{-3} \mathrm{pc} \mathrm{cm}^{-3} \mathrm{yr}^{-1}\right)\end{array}$ & $\begin{array}{l}\text { Amplitude } \\
\left(10^{-4} \mathrm{pc} \mathrm{cm}^{-3}\right)\end{array}$ & $\begin{array}{l}\text { Period } \\
\text { (days) }\end{array}$ & $\chi_{r}^{2}$ & Start & End & $\begin{array}{r}\text { Length } \\
\text { (days) }\end{array}$ & $\begin{array}{r}\delta t \\
\text { (days) }\end{array}$ \\
\hline \multirow[t]{2}{*}{ J0613-0200 } & Both & $0.066(7)$ & $1.8(1)$ & $358(4)$ & 0.72 & 53448 & 54970 & 3137 & 18 \\
\hline & Both & $0.161(7)$ & $1.2(2)$ & $352(5)$ & 3.5 & 54970 & 56380 & - & 23 \\
\hline \multirow[t]{2}{*}{ J1600-3053 } & Linear & $-0.73(4)$ & - & - & 2.8 & 54400 & 55300 & 2184 & 40 \\
\hline & None & - & - & - & - & 55300 & 56585 & - & - \\
\hline \multirow[t]{2}{*}{$\mathrm{J} 1640+2224$} & Linear & $0.145(3)$ & - & - & 7.0 & 53344 & 55850 & 3254 & 78 \\
\hline & None & - & - & - & - & 55850 & 56599 & - & - \\
\hline \multirow[t]{3}{*}{$\mathrm{J} 1713+0747$} & Both & $-0.066(8)$ & $0.5(1)$ & $400(16)$ & 2.0 & 53393 & 54730 & 3199 & 38 \\
\hline & None & - & - & - & - & 54730 & 54900 & - & - \\
\hline & Both & $-0.015(5)$ & $0.52(9)$ & $369(7)$ & 5.5 & 54900 & 56592 & - & 26 \\
\hline \multirow[t]{2}{*}{ J1918-0642 } & Both & $-0.49(1)$ & $1.2(4)$ & $385(11)$ & 4.3 & 53292 & 56000 & 3293 & 24 \\
\hline & Both & $0.23(3)$ & $1.2(3)$ & $541(47)$ & 2.9 & 56000 & 56585 & - & 31 \\
\hline \multirow[t]{3}{*}{ B1937+21 } & Both & $-0.34(3)$ & $3.2(4)$ & $395(11)$ & 28 & 53267 & 54550 & 3327 & 5 \\
\hline & None & - & - & - & - & 54550 & 55970 & - & - \\
\hline & Both & $0.050(3)$ & $3.7(2)$ & $469(14)$ & 10 & 55970 & 56594 & - & 5 \\
\hline
\end{tabular}

$\overline{\text { Notes. }}$ Results of fitting periodic and linear trends to the DM variations, where $1 \sigma$ uncertainty in the last significant digit is expressed in parentheses. The upper section lists pulsars where a single fit was applied; columns are the detected trend, the slope, the amplitude of the periodic fit, the period of the fit, the reduced chi-squared after the fitting, the period found by the Lomb-Scargle periodogram, the false alarm probability for that period, the length of the data span for that pulsar, and the average time it takes the DM time series to vary by $1 \sigma$. The lower section contains pulsars where piecewise trends were applied. The two columns that differ from above give the final start and end dates used in the fit. 
pulsar, a wedge with linear density changes in the ISM or the orbital motion of the Earth, among others; the possible geometries from which these trends arise are explained in detail in Lam et al. (2016). Both linear and periodic trends have been seen in Parkes Pulsar Timing Array (PPTA) data (You et al., 2007; Keith et al., 2013; Petroff et al., 2013; Reardon et al., 2016). Petroff et al. (2013) determine the significance of a linear trend by calculating the error of a fit to the slope; linear trends were deemed significant if the errors are less than $35 \%$ of the slope value and highly significant if the errors on the slope measurement are less than $20 \%$. This method is not applicable for the NANOGrav 9-year data set as a large number of pulsars exhibit sinusoidal trends without linear trends.

In order to determine the scale and structure of the variations, the options being linear, periodic, both, or variations consistent with stochastic noise, we applied a non-linear least squares fit to the data using three functions,

$$
\begin{aligned}
& \overline{\mathrm{DM}_{1}}(t)=m t+b, \\
& \overline{\mathrm{DM}_{2}}(t)=A \cos (\omega t+\phi)+b, \\
& \overline{\mathrm{DM}_{3}}(t)=A \cos (\omega t+\phi)+m t+b,
\end{aligned}
$$

with the $\chi_{r}^{2}$ calculated for the time series after each of these fits was individually subtracted off. For each fit, $N_{\text {DOF }} \approx N-N_{p}$, where $N$ is the number of DM measurements and $N_{p}$ is the number of free parameters being fit in that function $\left(N_{p}=1\right.$ when $\overline{\mathrm{DM}}(t)$ is a constant value). The three $\chi_{r}^{2}$ values were then compared to the original value; the result producing the lowest $\chi_{r}^{2}$ was assigned as the trend. 
The results of these fits can be seen in Table 2.2. There is a known complication when estimating the number of degrees of freedom for a non-linear model (Andrae et al., 2010). The $\chi_{r}^{2}$ is only used as a metric to compare the fits of models we know to be incomplete; as stated earlier, the ISM is more complicated than a purely linear trend plus annual component. The fitting routine incorporates a non-linear least squares fit which is locally linearized around the minimum $\chi_{r}^{2}$. Later on, we describe $\chi_{r}^{2}$ surfaces in the full parameter space and analyze the degree of covariance between fit parameters, finding it agrees with this fitting routine.

The periodic term in the function was fit using an initial guess of 365 days. Due to a change in sign of $d \mathrm{DM} / d t$ or the appearance/disappearance of a trend partway through the data span, PSRs J0613-0200, J1600-3053, J1640+2224, J1918-0642, and B1937+21 are not well characterized by a single fit. These MSPs were fit using piecewise functions, using the $\chi_{r}^{2}$ of the fit to identify the applicable MJD range for each fit. The results of this partial fitting can be seen in Table 2.2. The fits can be seen in Figures 2.1 through 2.5. The $\chi_{r}^{2}$ values listed in Table 2.2 are for the individual fit regions; these differ from the values shown in Figures 2.1 through 2.5 because those values incorporate both fits as well as any regions excluded from the fit.

We applied a Lomb-Scargle periodogram analysis to corroborate the best-fit periods, as an annual period was suggested during the trend fitting routine. The periodogram is also useful in possibly identifying non-annual periodicities (also seen in Table 2.2). This analysis is able to detect periodicities in unevenly sampled data (Scargle, 1982) for which a false alarm probability (FAP) may be calculated. The 

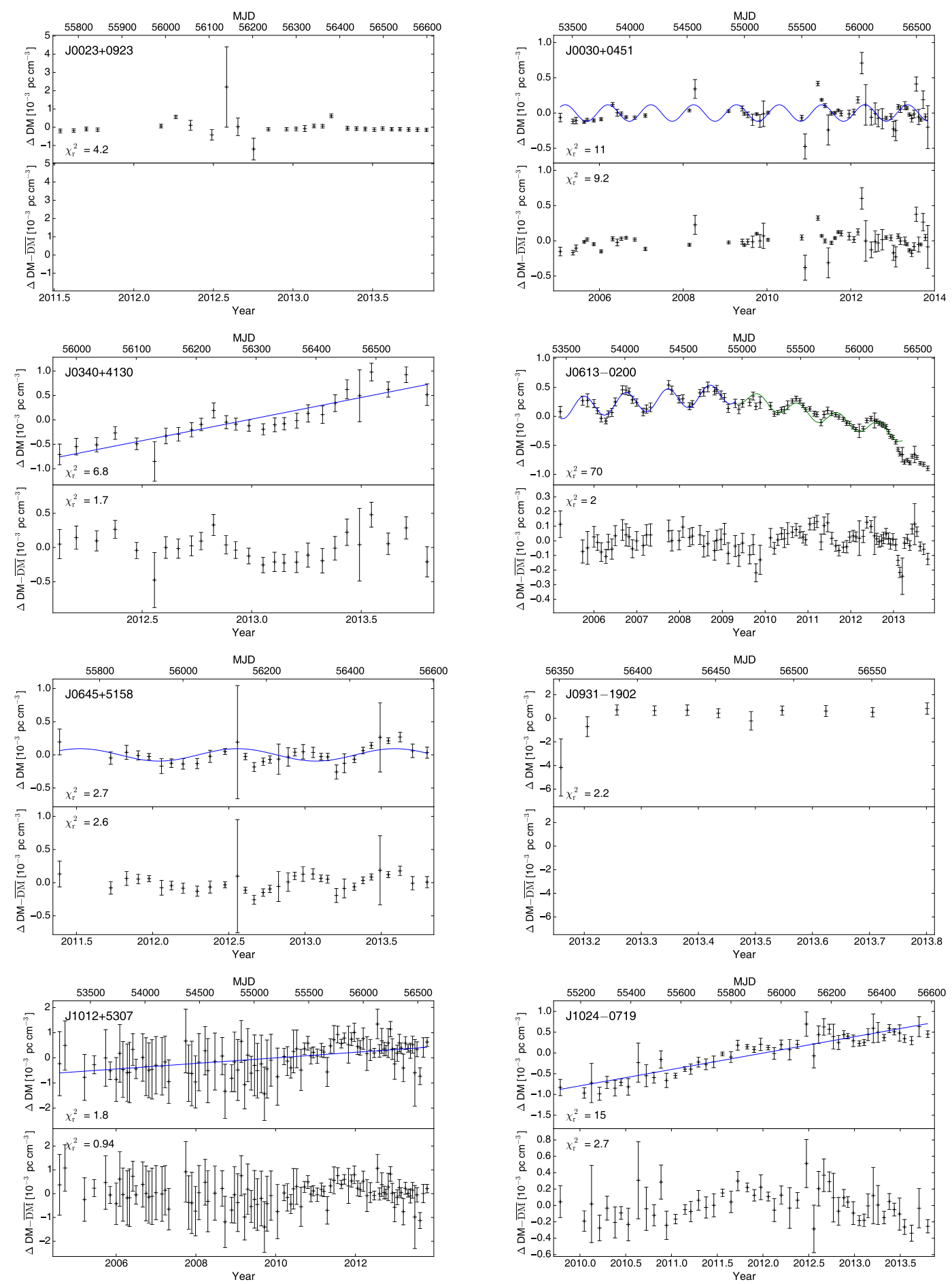

Figure 2.1 The top panel shows the DM time series with the best fit function (if applicable) in blue. The bottom panel shows the DM residuals after the trend has been removed from the time series; an empty panel means no trend was found. The $\chi_{r}^{2}$ values before and after these fits for each pulsar appear in the top and bottom panels respectively, as well as in Tables 2.1 and 2.2. PSR J0931-1902 has too short a data span for a trend to be determined. 

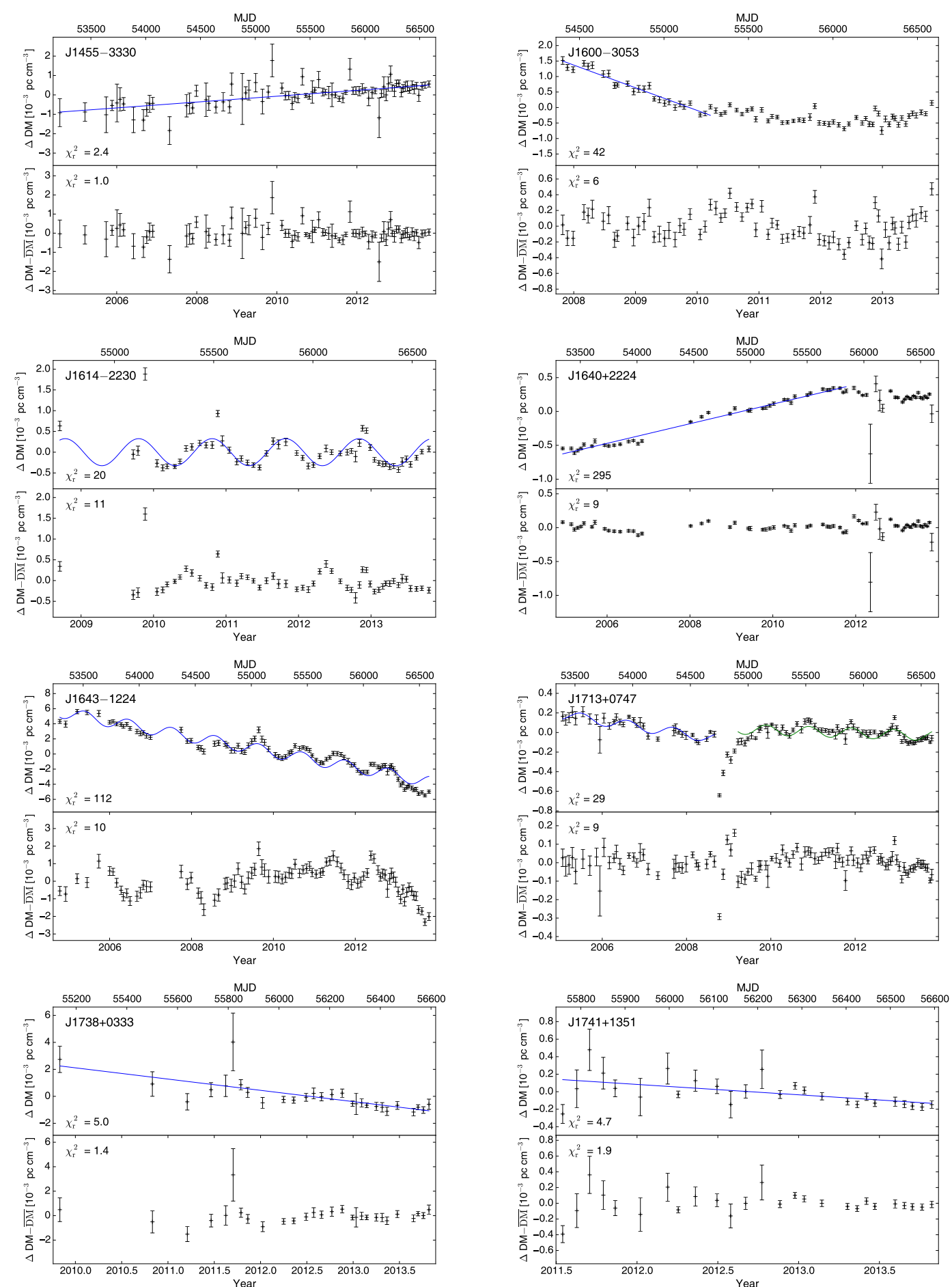

Figure 2.2 The top panel shows the DM time series with the best fit function (if applicable) in blue. The bottom panel shows the DM residuals after the trend has been removed from the time series; an empty panel means no trend was found. PSRs J1600-3053 and J1640+2224 were not found to have significant trends in the later parts of the DM time series. 

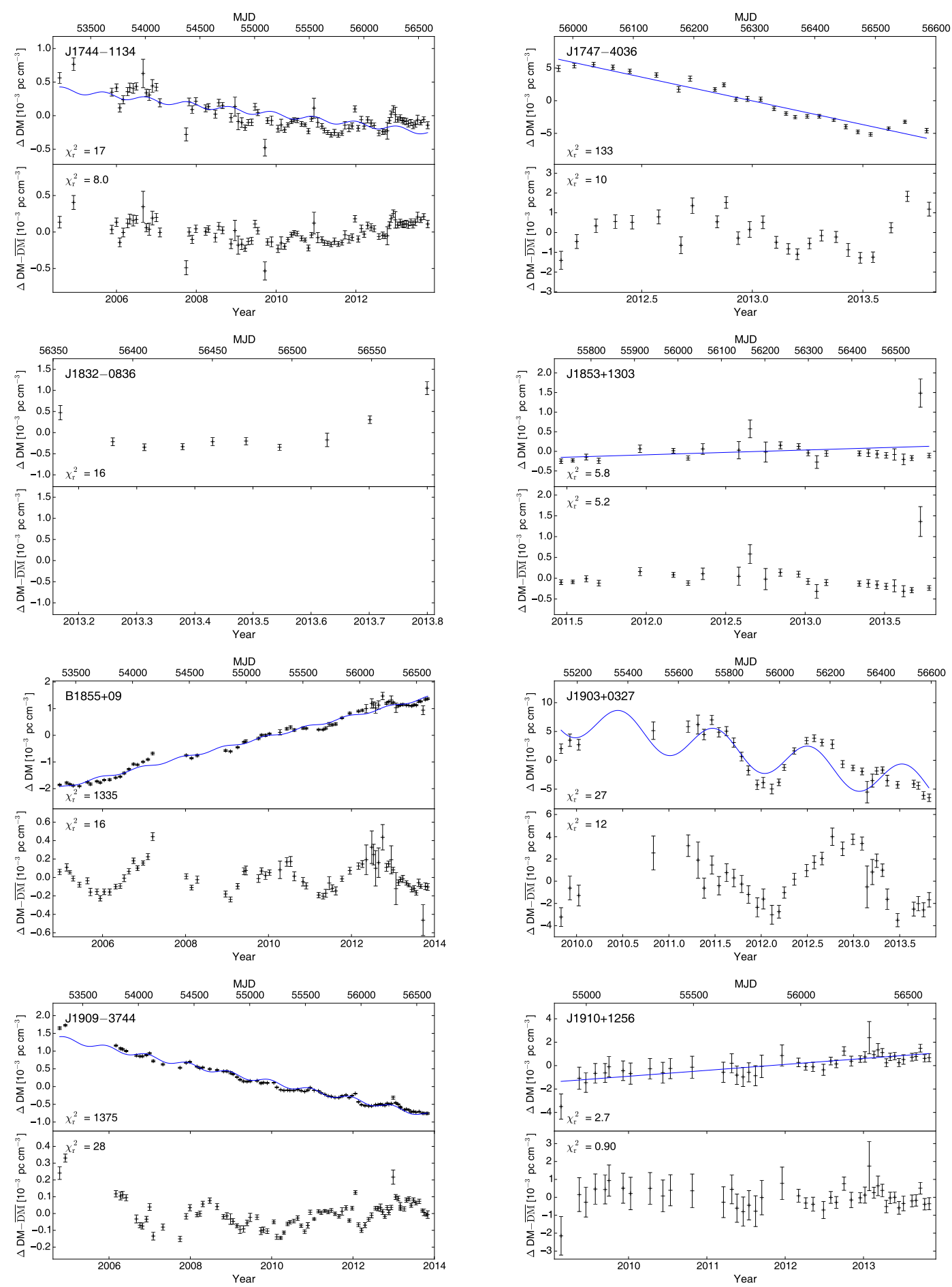

Figure 2.3 The top panel shows the DM time series with the best fit function (if applicable) in blue. The bottom panel shows the DM residuals after the trend has been removed from the time series; an empty panel means no trend was found. PSR J1832-0836 has too short a data span for a trend to be determined. 

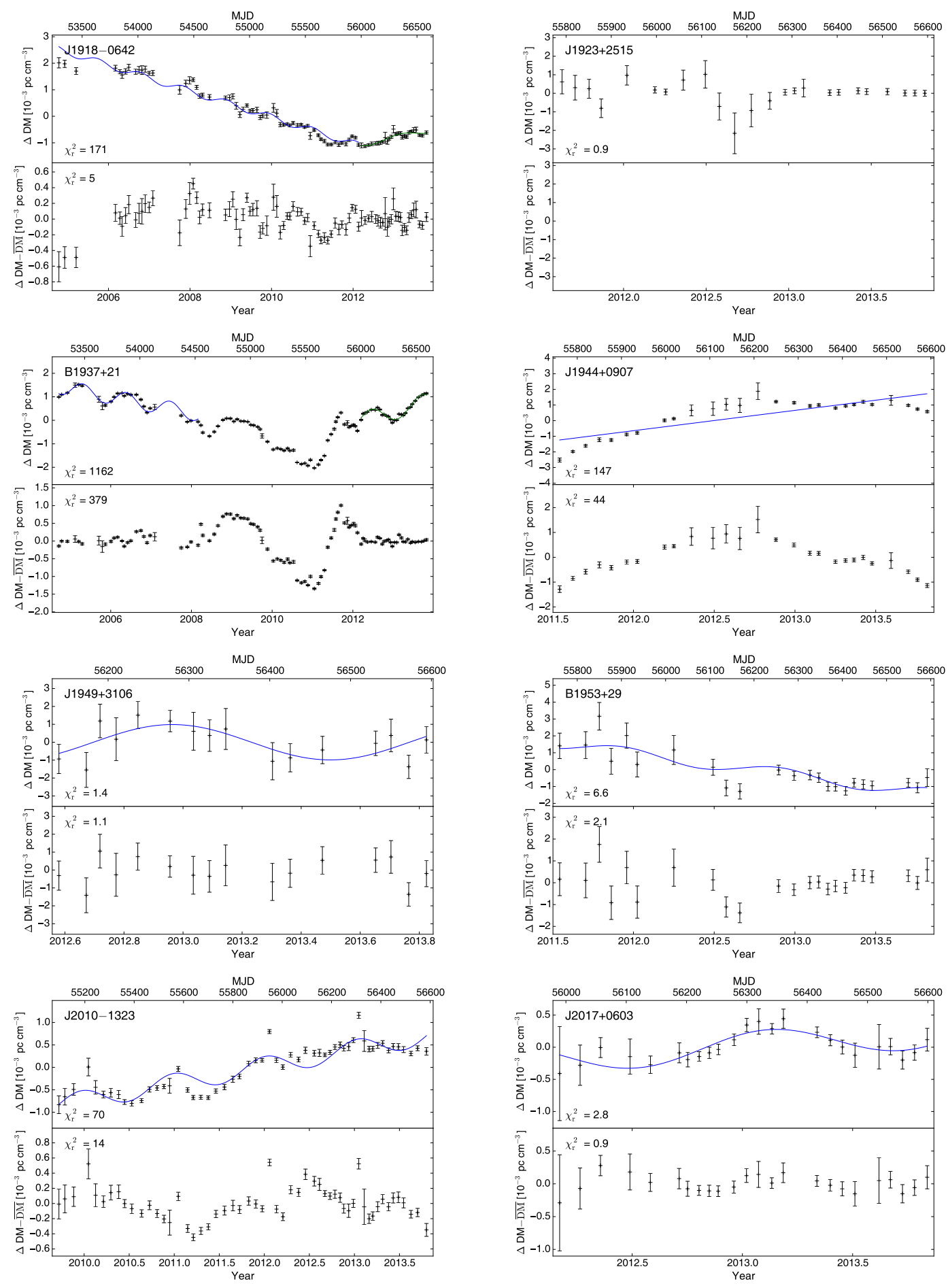

Figure 2.4 The top panel shows the DM time series with the best fit function (if applicable) in blue. The bottom panel shows the DM residuals after the trend has been removed from the time series; an empty panel means no trend was found. PSR B1937+21 could not be fit with a periodic trend throughout the data set. 

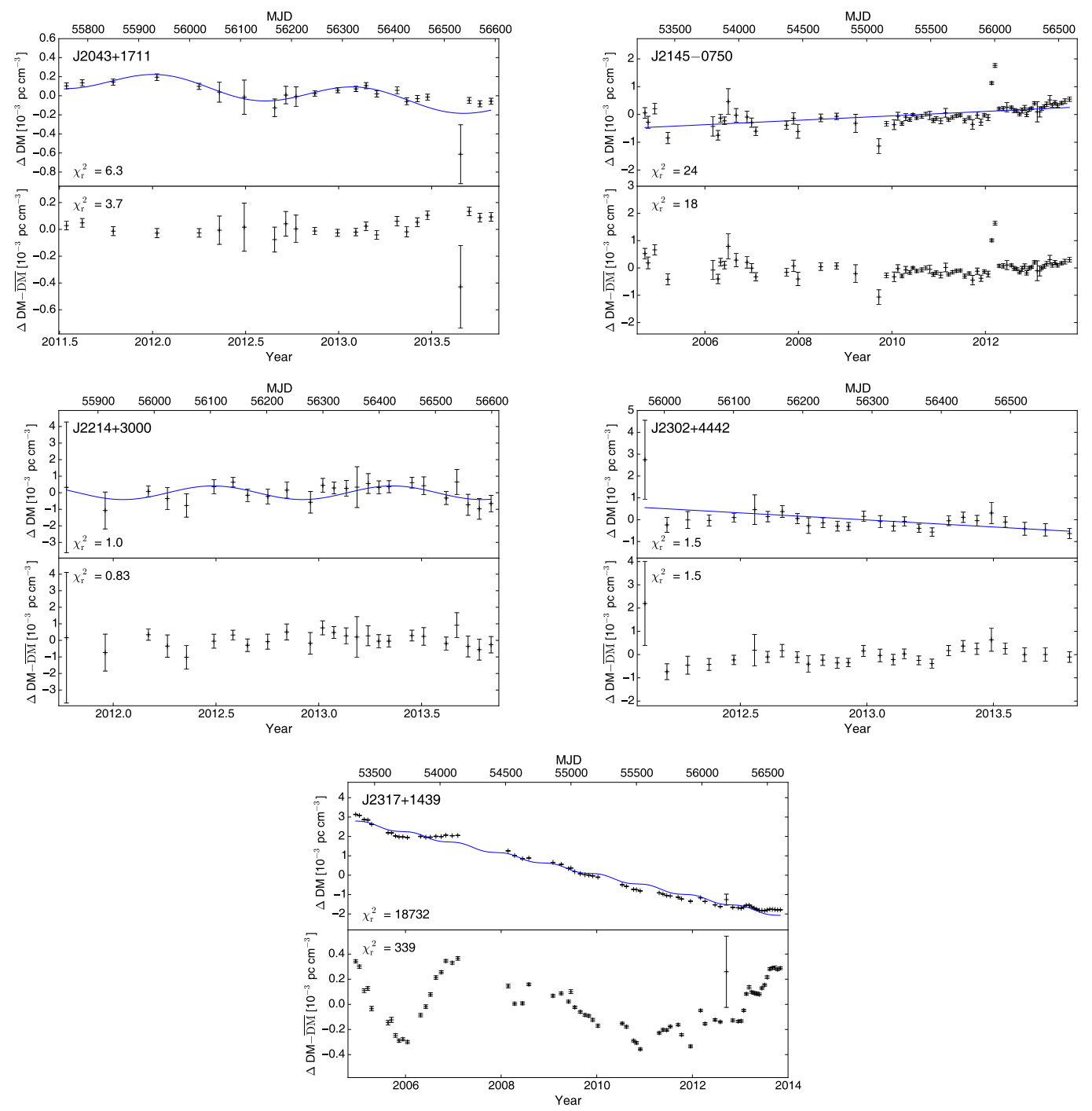

Figure 2.5 The top panel shows the DM time series with the best fit function (if applicable) in blue. The bottom panel shows the DM residuals after the trend has been removed from the time series; an empty panel means no trend was found. 
FAP is the likelihood that these periods would occur as a result of random white noise. We ignored periods found by the periodogram that coincided with either the length of the data set or the observing cadence. The resolution of the analysis is equal to the cadence of the observations. Any linear trend in the DM variations will mask the periodic effect, and therefore was removed from those identified to have linear effects before applying the periodogram analysis.

\subsubsection{DM variation timescale}

The DM value can vary on timescales of years, days, or even hours. Therefore it is important to know on what timescale this DM is accurate. The time $\delta t$ for DM to change by $\sigma_{\mathrm{DM}}$, the rms DM in the DM time series (prior to fitting any DM trends), gives us a rough estimate for how long a single DM estimation is valid. For a linear trend

$$
\frac{\sigma_{\mathrm{DM}}}{\delta t}=\frac{d \mathrm{DM}}{d t}=m \longrightarrow \delta t=\frac{\sigma_{\mathrm{DM}}}{m}
$$

where $m$ is the slope of the linear trend, seen also in Equation 2.4. The time associated with a periodic trend

$$
\frac{\sigma_{\mathrm{DM}}}{\delta t}=\frac{d \mathrm{DM}}{d t} \approx A \omega \longrightarrow \delta t=\frac{\sigma_{\mathrm{DM}}}{A \omega}
$$

where $A$ and $\omega$ are the amplitude and angular frequency of the periodic trend respectively. The variation time for a DM time series showing both trends combines 

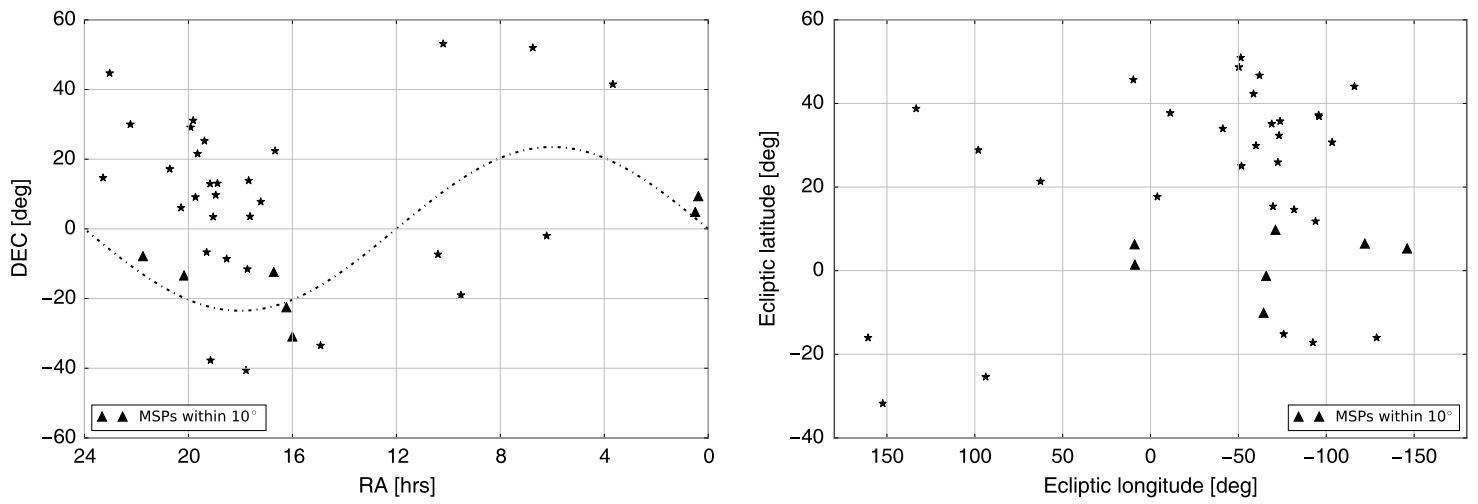

Figure 2.6 MSP positions with respect to the ecliptic, shown in RA and Dec (left) as well as ecliptic coordinates (right). In the left plot, the ecliptic is depicted by the dashed line. Sources that lie within $\sim 10^{\circ}$ of the ecliptic are signified by triangles.

the $d \mathrm{DM} / d t$ components from both the periodic and linear components

$$
\frac{\sigma_{\mathrm{DM}}}{\delta t} \approx m+A \omega \longrightarrow \delta t \approx \frac{\sigma_{\mathrm{DM}}}{m+A \omega}
$$

The $\delta t$ values for the MSPs showing trends are seen in Tables 2.2. This $\delta t$ can inform on what timescale our DM measurement is constant and the importance of observing at epochs with spacing smaller than this timescale.

\subsubsection{Solar-angle correlation}

Pulsars that lie close to the ecliptic (within approximately $10^{\circ}$ ) will have their LOS pass near the Sun during Earth's orbit. This proximity can cause a sinusoidal trend in DM variations due to the variation in $n_{e}$ along the LOS from the solar wind.

We examine the pulsar positions with respect to the ecliptic to determine for which MSPs this effect could be significant. As can be seen in Figure 2.6, 

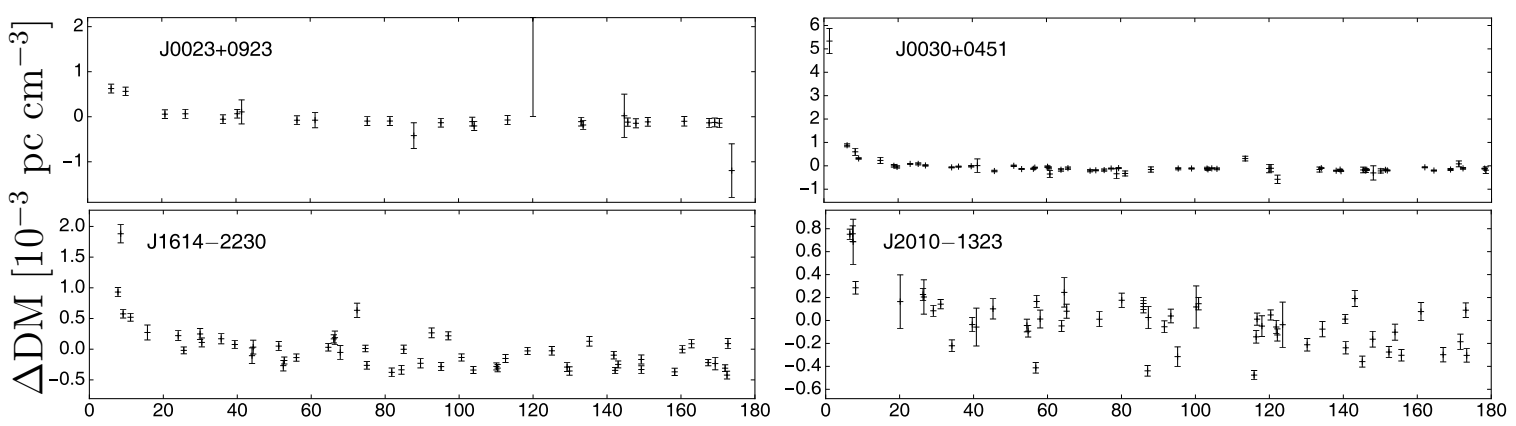

Solar Elongation (degrees)

Figure 2.7 DM variations with respect to the solar position angle. The linear trend identified for PSR J2010-1323 has been subtracted in order to better identify any correlation in the DM as a function of solar angle. Note that the two highest DM points seen for PSR J0030+0451 were omitted from the 9-year data set as outliers and are not plotted in Figure 2.1 (discussed in Section 2.4.3).

PSRs J0023+0923, J0030+0451, J1614-2230, and J2010-1323 reside close (within approximately $6.3^{\circ}, 1.5^{\circ}, 6.8^{\circ}$, and $6.5^{\circ}$ respectively on closest approach in the data set) to the ecliptic. The DM as a function of solar elongation angle can be seen in Figure 2.4.3. PSRs J0023+0923 and J2010-1323 show a slight peak in DM at the smallest pulsar-Sun angles. PSRs J0030+0451 and J1614-2230 show significant peaks at the minimal solar elongation angle. It should be noted that the two highest DM points for J0030+0451 were omitted from Arzoumanian et al. (2015) as outliers but were included for this portion of the analysis.

\subsubsection{Pulsar trajectories}

We have plotted the pulsar trajectories through the ISM as seen from Earth, color coded to signify the DM value at each epoch (Figures 2.8 through 2.11). For this, we assumed that all of the free electrons along the line of sight are sitting in 
a stationary phase screen located halfway between the Earth and the pulsar. The trajectories are the projected motions of the pulsar as seen on this phase screen. Using proper motion and distance estimates with errors from the NE2001 model (Cordes \& Lazio, 2002), the transverse velocity can be calculated and used to track the pulsar's trajectory in the sky. Proper motions were taken from the data release (seen in Table 2.1). These trajectory maps can be useful in isolating features in the ISM as well as visualizing trends in the DM time series.

\subsection{Structure functions}

Turbulence in the ISM is typically described as having a Kolmogorov power spectrum, meaning we expect to find larger variations over longer timescales. The power spectrum used to derive the structure function has the form

$$
P(q) \propto q^{-\beta}, q_{\text {outer }} \leq q \leq q_{\text {inner }}
$$

where $q$ is the reciprocal of the size scale, and $\beta$ is the power spectrum exponent. A Kolmogorov medium corresponds to a $\beta$ value of $11 / 3$, while the highest value expected for turbulence in the ISM (for an inner scale shorter than $10^{9} \mathrm{~m}$ ) is $\beta=4$ (Rickett, 1990). The outer scale is described as the size at which the ISM ceases to be homogeneous, and the inner scale is the point at which dissipation occurs in the material along the line of sight.

The DM structure function $(\mathrm{SF})$ is an effective analytic tool for characterizing interstellar turbulence over various time and size scales (Rickett, 1990; Cordes et al., 

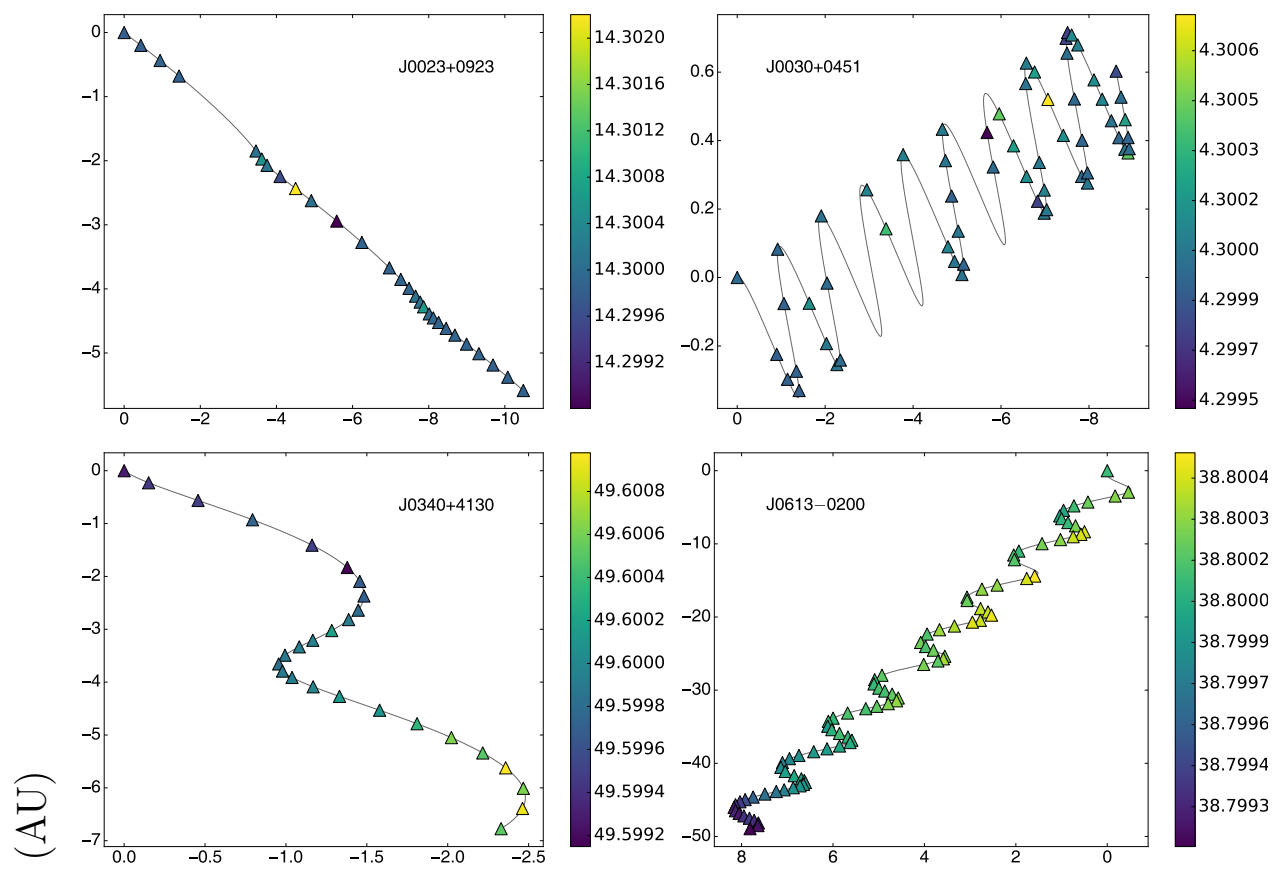

$\lambda$
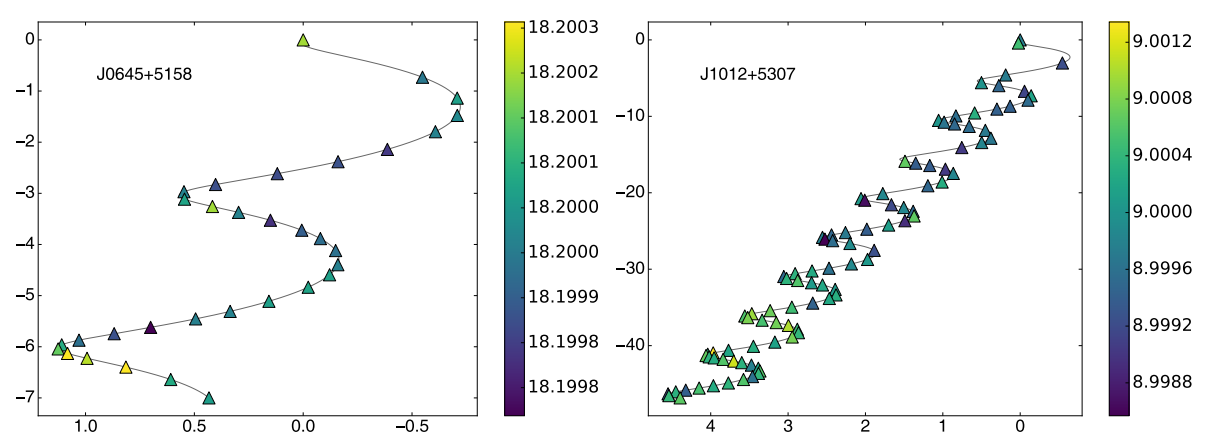

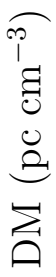
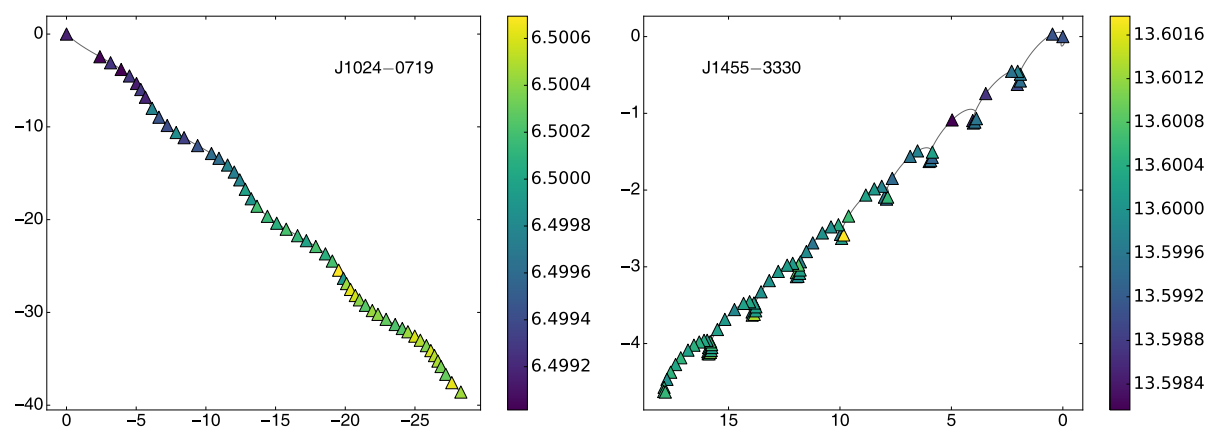

$\Delta_{X}(\mathrm{AU})$

Figure 2.8 MSP trajectories are plotted with color mapping the DM at each epoch. PSR J0613-0200 shows higher DM regions on one side of its trajectory over time, suggesting the presence of a DM gradient that is transverse compared to the pulsar's motion. PSR J1024-0719 shows a monotonically increasing DM, which could be due to a DM gradient oriented in the same direction as the pulsar's motion. 

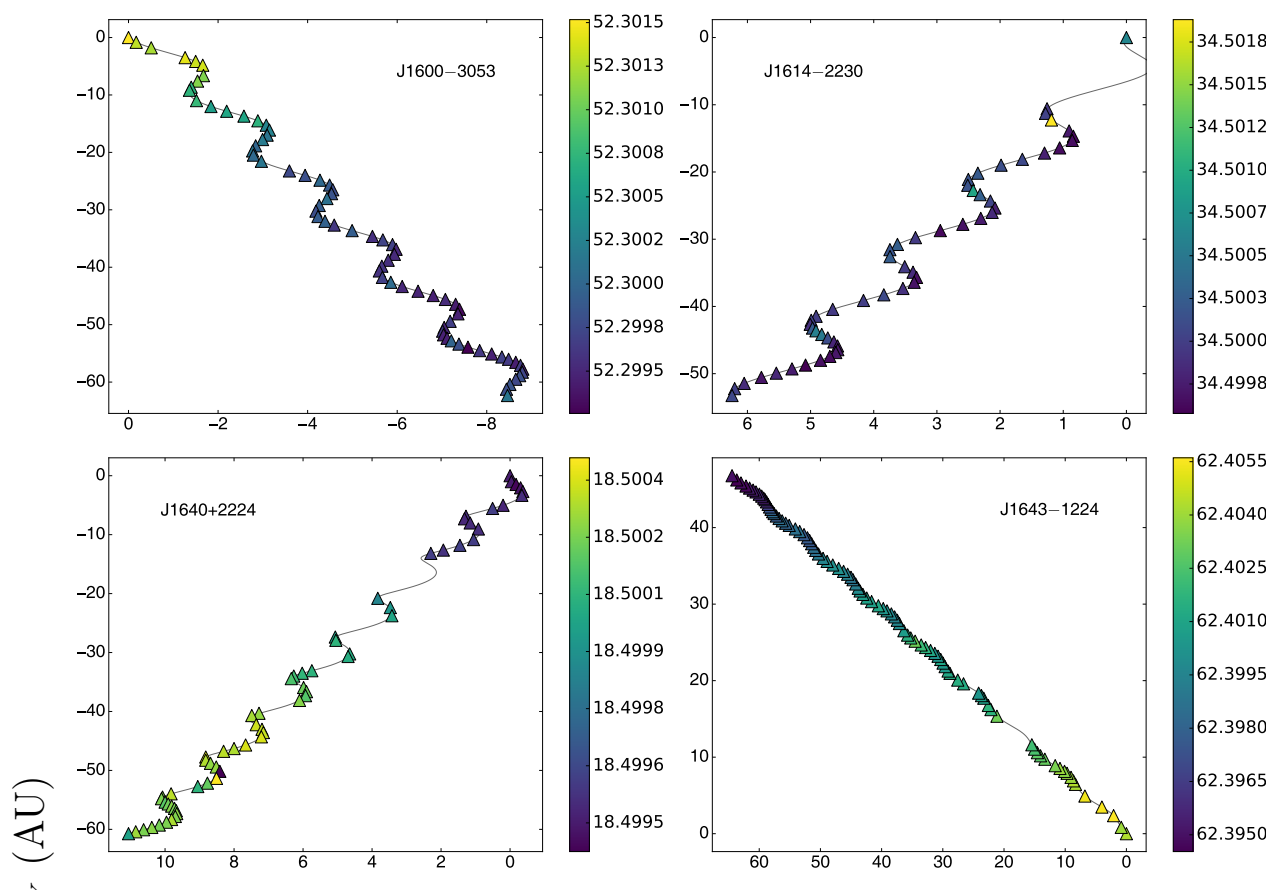

y
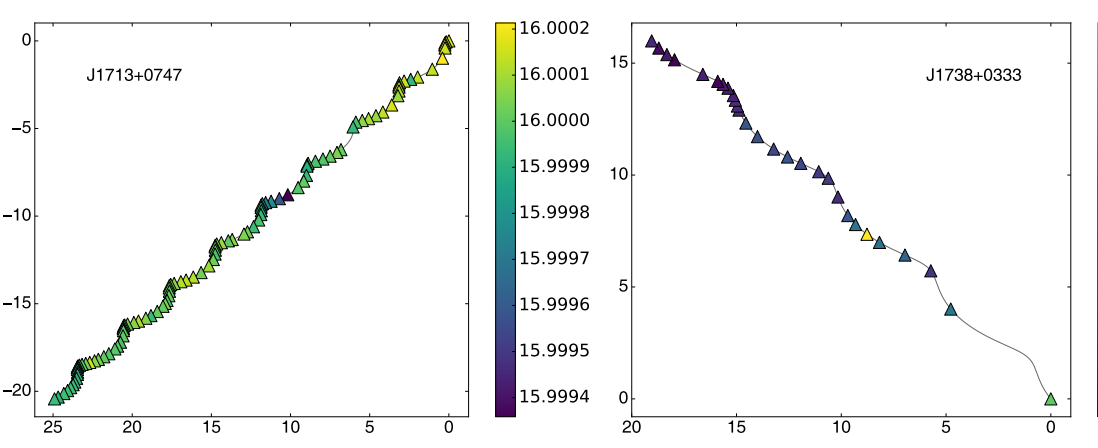

$-33.8038$

$-33.8032$

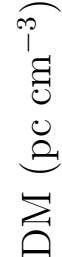
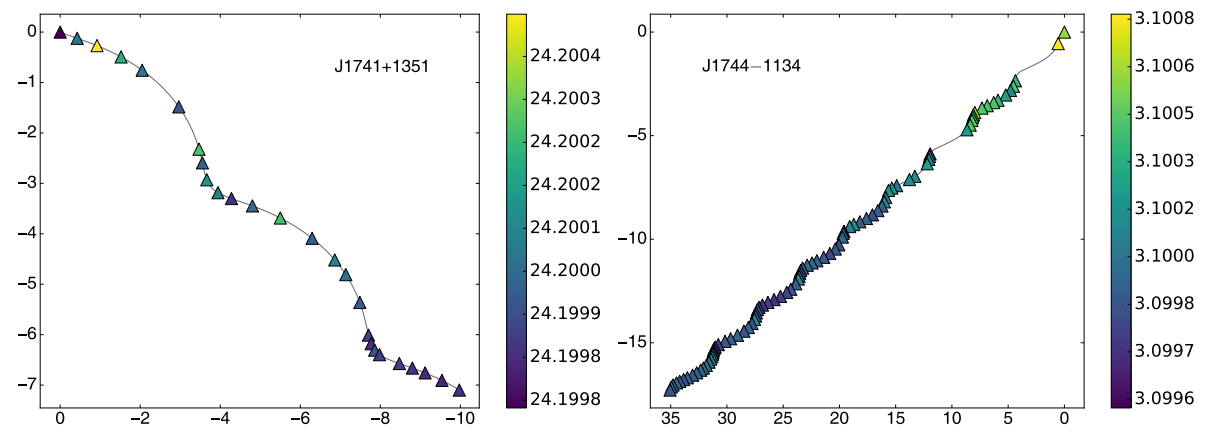

\section{$\Delta_{X}(\mathrm{AU})$}

Figure 2.9 MSP trajectories are plotted with color mapping the DM at each epoch. PSRs J1600-3053, J1640+2224, and J1643-1224 show a monotonically increasing or decreasing DM, which could be due to a DM gradient along the direction of motion. PSR J1614-2230 has high DM regions in the same part of its trajectory every year, suggesting a localized overdensity along the line of sight to the pulsar. 

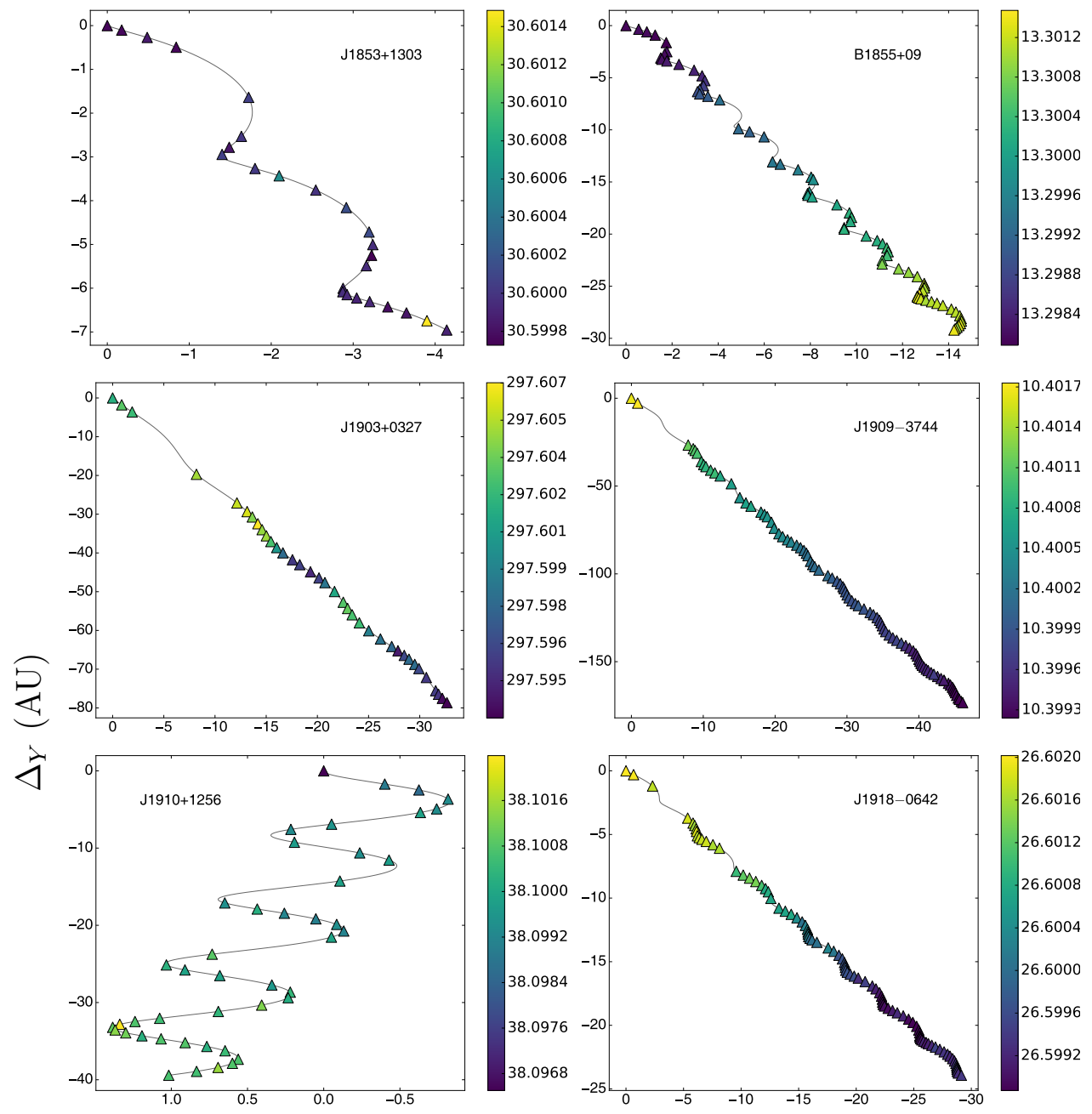

10.4014

10.4011

10.4008

10.4005

10.4002

10.3999

0
1
0
0
$\Xi$
$己$
$\vdots$

26.6012

26.6008

26.6004

26.6000

26.5996

26.5992
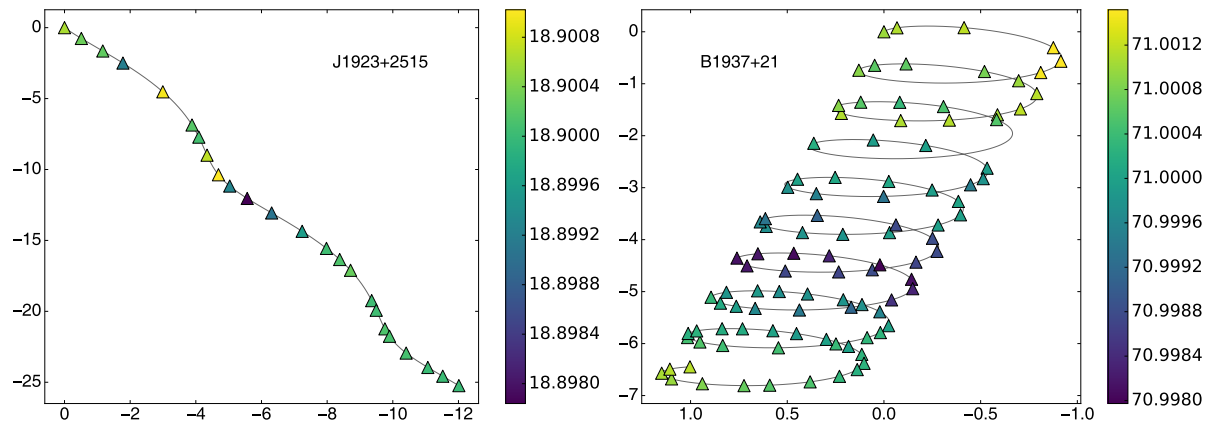

$\Delta_{X}(\mathrm{AU})$

Figure 2.10 MSP trajectories are plotted with color mapping the DM at each epoch. PSRs B1855+09, J1909-3744, and J1918-0642 show a monotonically increasing or decreasing DM, which could be due to a DM gradient along the direction of motion. PSR J1903+0327 has high DM regions in the same part of its trajectory every year, suggesting a localized overdensity along the line of sight to the pulsar. PSR B1937+21 shows a distinct low DM region that is also evident in the DM time series, indicative of a localized bubble in the ISM. 

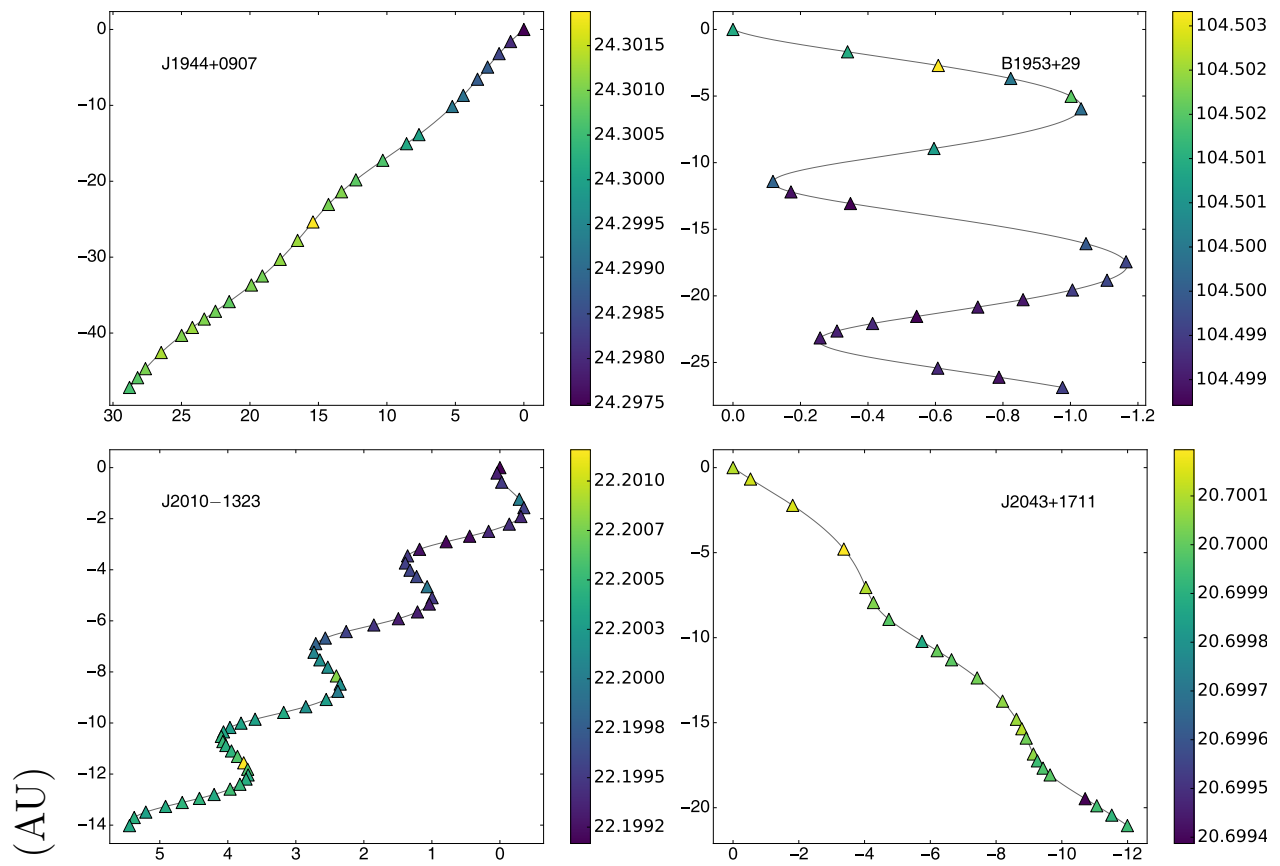

$\lambda$
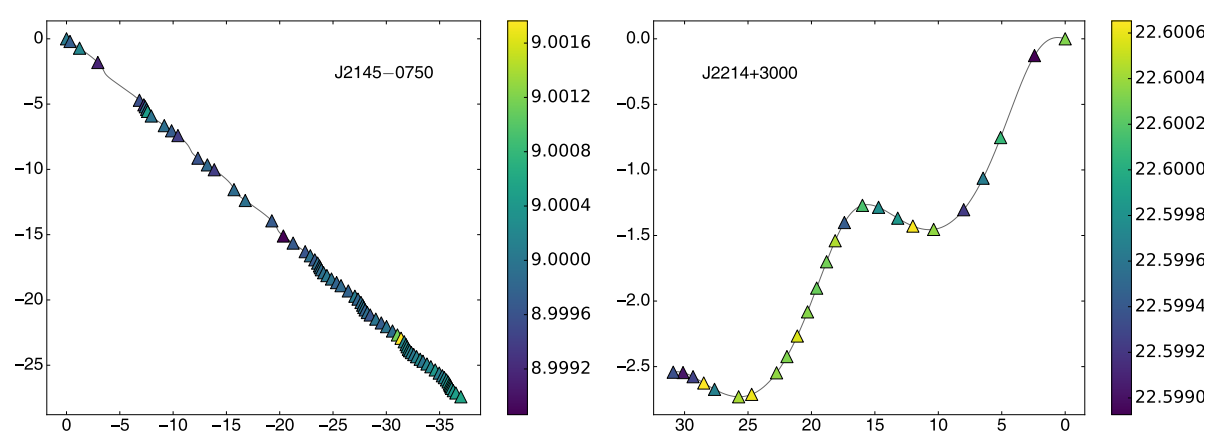

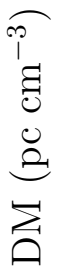

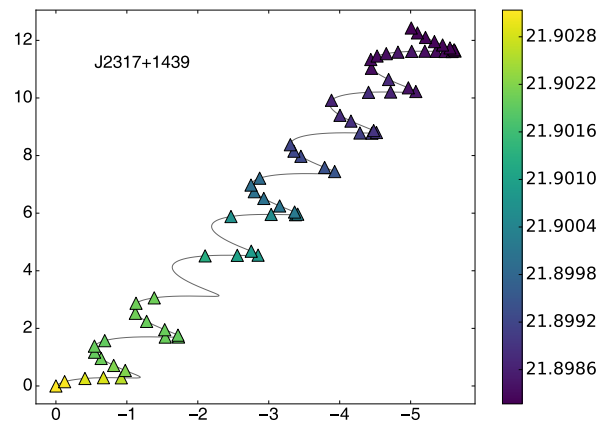

$\Delta_{X}(\mathrm{AU})$

Figure 2.11 MSP trajectories are plotted with color mapping the DM at each epoch. PSRs J1944+0907 and J2317+1439 show a monotonically increasing or decreasing DM, which could be due to a DM gradient along the direction of motion. PSR J2010-1323 shows an increasing DM in the direction of the pulsar's motion as well as high DM regions in the same part of its trajectory every year, suggesting a localized overdensity along the line of sight to the pulsar. 

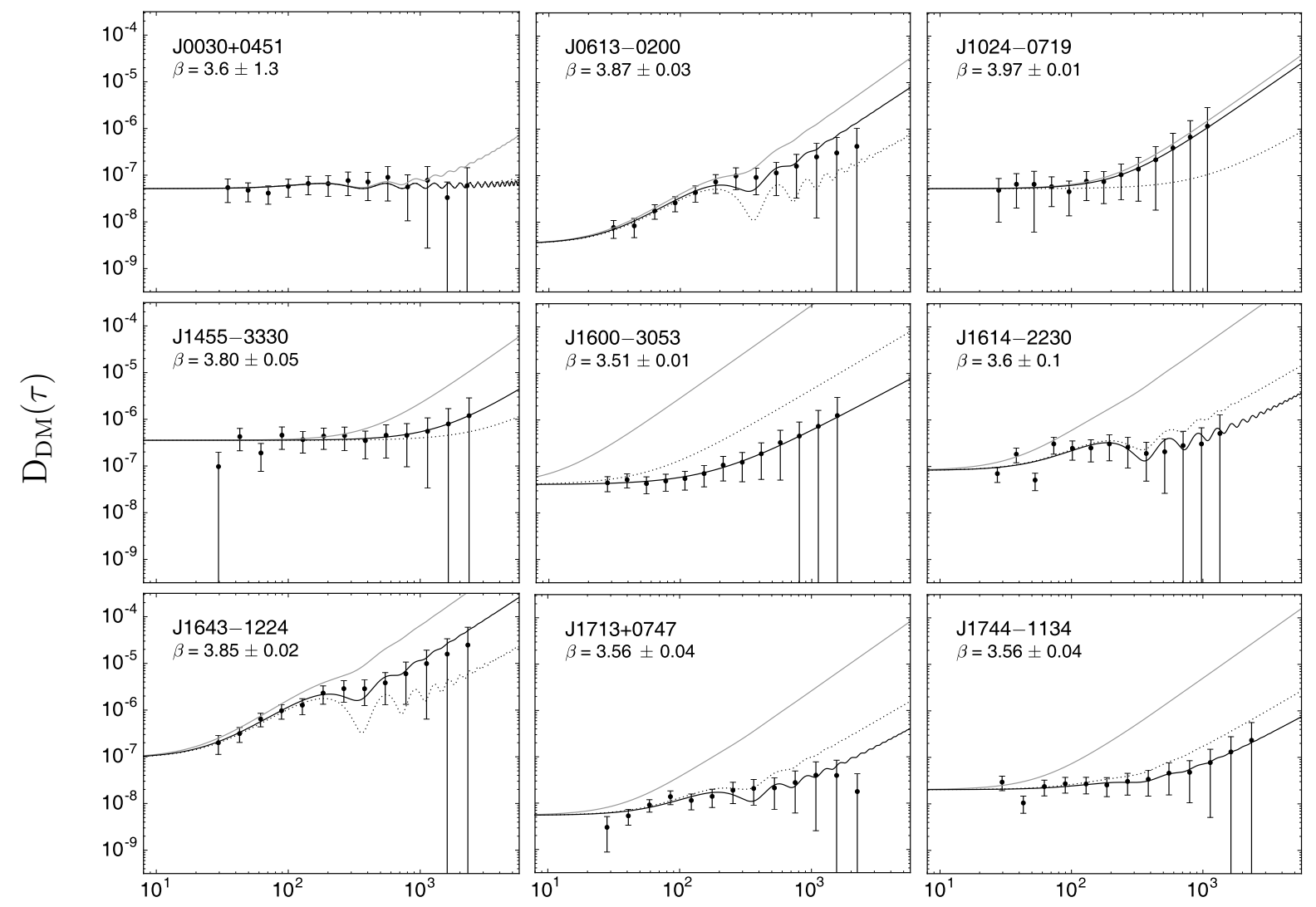

Time Lag [days]

Figure 2.12 Structure functions for the DM variations, calculated for the MSPs with measured diffractive timescales. Error bars extending to the bottom of the frame signify an upper limit (in agreement with You et al. (2007)). The solid grey lines signify a quadratic power law and the dotted lines signify a Kolmogorov power law, which are anchored to the diffractive timescale, while the solid black lines are the best fits for the model. The errors associated with $\beta$ are $\pm 1 \sigma$ errors. 

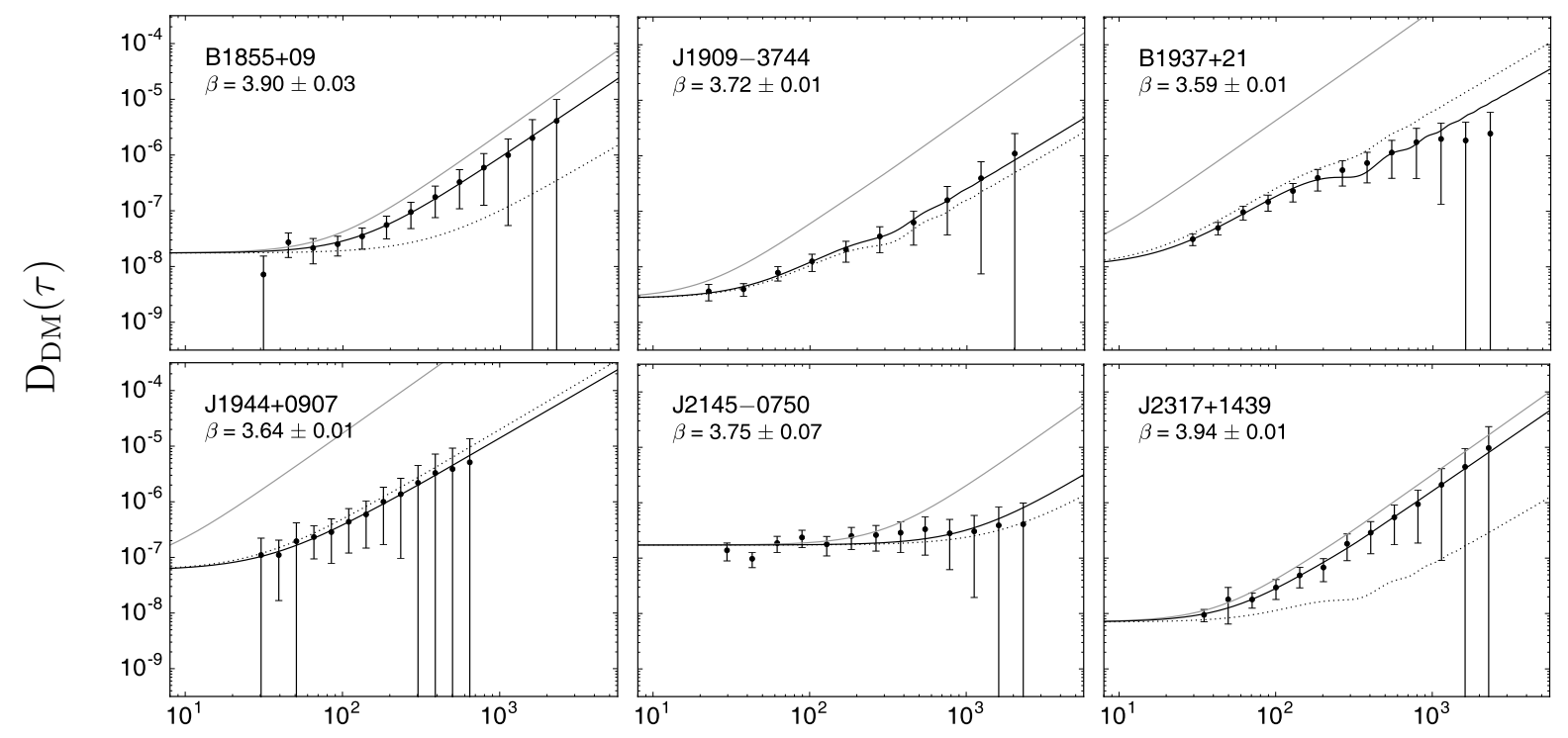

Time Lag [days]

Figure 2.13 Structure functions for the DM variations, calculated for the MSPs with measured diffractive timescales. Error bars extending to the bottom of the frame signify an upper limit (in agreement with You et al. (2007)). The solid grey lines signify a quadratic power law and the dotted lines signify a Kolmogorov power law, which are anchored to the diffractive timescale, while the solid black lines are the best fits for the model. The errors associated with $\beta$ are $\pm 1 \sigma$ errors. 
1990; You et al., 2007; Keith et al., 2013; Fonseca et al., 2014; Lam et al., 2016). We compute SFs by binning the change in time across all epochs into equally log-spaced bins after calculating

$$
D_{\mathrm{DM}}(\tau)=\left\langle[\mathrm{DM}(t+\tau)-\mathrm{DM}(t)]^{2}\right\rangle
$$

where $\tau$ is the time lag in days (Cordes \& Rickett, 1998). The diffractive timescale $\Delta \tau_{D}$, the scale during which the diffraction intensity varies as a result of irregularities in the ionized plasma along the line of sight, is used to anchor the SF

$$
D_{\mathrm{DM}}(\tau)=\left(A_{\nu} \nu\right)^{2}\left(\tau / \Delta \tau_{\mathrm{D}}\right)^{\beta-2}
$$

where $A_{\nu}=3.84 \times 10^{-5} \mathrm{MHz}^{-2} \mathrm{~s}^{-1} \mathrm{pc} \mathrm{cm}{ }^{-3}$ and $\nu$ is the observing frequency. Epoch to epoch variations of $\Delta \tau_{\mathrm{D}}$ are expected of order $10 \%$ or more (Lam et al., 2016). The SF is poorly estimated at large time lags, and so some functions may appear Kolmogorov at shorter timescales but fall below at longer time lags; this is an indication of an underlying Kolmogorov spectrum (You et al., 2007). This is also why SF values at high time lags may have large errors on them. Quadratic SFs occur when the time lag is smaller than the timescale necessary to adequately probe the structure in a region, if any (Lam et al., 2016).

Several models were applied to the $\mathrm{SF}$ in order to constrain a range for $\beta$. Fitting only for Equation 2.10 is problematic because there are other contributions to the SF, such as trends and noise, among other possibilities. However, over-fitting 
the SF will cause the fit to fail for a number of reasons, discussed in this section. The models are of the form

$$
D_{\mathrm{DM}}(\tau)=D_{\text {sto }}(\tau)+D_{\text {lin }}(\tau)+D_{\text {per }}(\tau)+D_{\text {noise }}(\tau)
$$

where the first term is the stochastic (e.g. from the electron density wave number spectrum) component, the second term is the linear component, the third term is the periodic component, and the last term is the noise (e.g. measurement errors) component.

One method is to fit the stochastic and noise components while fixing the linear trend and periodic components to the trend values found in the time series analysis. However, we were only able to successfully fit the SF for one pulsar (PSR J1643-1224) using this model. In all other cases, the SF of the two trends is higher than the calculated SF; fitting for a stochastic component on top of that would only increase the chi-squared, and the fit fails. This failure is evidence that there is contamination between the stochastic and trend components. In many cases, a linear trend fit over the time series will absorb part of the stochastic component. Therefore, the "true" linear trend may be different than the one we fit for in the time series, which will bias the component in the SF high. Therefore, we are unable to obtain a proper fit using this model.

To bypass this contamination between the trend and stochastic aspects, we tried fitting for all parameters (stochastic, noise, linear, and periodic) without using any prior information in the hopes of allowing a fit and comparing values with those 
previously found. Due to the covariant nature of the parameters, we found values for the stochastic, periodic amplitude, and noise components, but the amplitude of the periodic trend was not much larger than its uncertainties while the linear trend component was found to be zero. The period was then fixed to one year in order to eliminate some of the covariance; fixing the period again gave significant values for the stochastic, noise, and periodic amplitude components, and no significance for an additional linear component. This is further evidence of the high covariance between the stochastic and linear trend components. The periodic amplitudes found here agree very well with those found previously by the trend analysis. Simultaneously fitting for both the stochastic and linear components does not appear to yield significant values for both.

The simplest model is then to only fit for stochastic and noise components while not fitting for a linear trend component. This model can be applied to all pulsars. Because the periodic component was not found to be highly covariant with the stochastic component, the periodic amplitudes were fitted as well and compared to the values from the trend analysis. In order to constrain a linear trend, we would need some prior information on the shape of the stochastic component in the time series relative to the true linear component that we do not have at this time.

Diffractive timescales, listed in Table 2.3, were calculated by creating a 2dimensional dynamic spectrum of each $1500-\mathrm{MHz}$ observation in the data set and computing the 2D autocorrelation function of each spectrum, which in turn is summed over time and frequency separately. A Gaussian function is fitted to the 1D frequency-summed autocorrelation function, and the scintillation timescale is 
Table 2.3 Diffractive timescales for 18 MSPs

\begin{tabular}{|c|c|c|c|c|c|c|}
\hline PSR & $\begin{array}{r}\nu_{\text {obs }} \\
(\mathrm{MHz})\end{array}$ & $\begin{array}{r}\Delta \tau_{\mathrm{D}} \\
(\min )\end{array}$ & Source & $\beta$ & $\begin{array}{r}\sigma_{n} \\
\left(10^{-4} \mathrm{pc} \mathrm{cm}^{-3}\right)\end{array}$ & $\begin{array}{r}A \\
\left(10^{-4} \mathrm{pc} \mathrm{cm}^{-3}\right)\end{array}$ \\
\hline $\mathrm{J} 0030+0451$ & 436 & 167.7 & 1 & $3.6(1.3)$ & $1.6(1)$ & $0.9(4)$ \\
\hline J0613-0200 & 1400 & 75 & 2 & $3.87(3)$ & $0.4(1)$ & $1.5(2)$ \\
\hline J1024-0719 & 1400 & 69.7 & 2 & $3.97(1)$ & $1.6(5)$ & 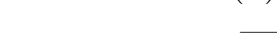 \\
\hline J1455-3330 & 436 & 17.7 & 3 & $3.80(5)$ & $3.8(4)$ & - \\
\hline \multirow[t]{2}{*}{ J1600-3053 } & 1400 & 4.5 & 2 & $3.51(1)$ & $1.43(3)$ & - \\
\hline & 1373 & 4.7 & 4 & & & \\
\hline \multirow[t]{2}{*}{ J1614-2230 } & 1400 & 12.5 & & $3.6(1)$ & $2.0(4)$ & $3.4(8)$ \\
\hline & 1500 & 8.1 & & & & \\
\hline J1643-1224 & 1400 & 9.7 & 2 & $3.85(2)$ & $2.2(6)$ & $8.9(7)$ \\
\hline \multirow[t]{3}{*}{$\mathrm{J} 1713+0747$} & 1400 & 47.6 & 2 & $3.56(4)$ & $0.52(8)$ & $0.7(2)$ \\
\hline & 430 & 14 & 4 & & & \\
\hline & 436 & 28 & 4 & & & \\
\hline \multirow[t]{3}{*}{ J1744-1134 } & 1400 & 34.5 & 2 & $3.56(4)$ & $1.01(9)$ & $0.5(4)$ \\
\hline & 436 & 21 & 4 & & & \\
\hline & 660 & 20 & 4 & & & \\
\hline \multirow[t]{2}{*}{ B1855+09 } & 1500 & 20.3 & & $3.90(3)$ & $0.93(7)$ & - \\
\hline & 1500 & 24.4 & 2 & & & \\
\hline \multirow[t]{2}{*}{ J1909-3744 } & 1300 & 30.6 & & $3.72(1)$ & $0.37(2)$ & $0.63(7)$ \\
\hline & 1400 & 37.6 & 2 & & & \\
\hline \multirow[t]{6}{*}{ B1937+21 } & 1500 & 4.0 & & $3.59(1)$ & $0.7(2)$ & $3.2(3)$ \\
\hline & 1500 & 7.4 & & & & \\
\hline & 320 & 1.1 & 4 & & & \\
\hline & 430 & 1.7 & 4 & & & \\
\hline & 1400 & 7.4 & 4 & & & \\
\hline & 1400 & 5.5 & 2 & & & \\
\hline J1944+0907 & 1500 & 2.0 & & $3.64(1)$ & $1.7(2)$ & - \\
\hline \multirow{3}{*}{$\mathrm{J} 2145-0750$} & 1400 & 56.6 & 2 & $3.75(7)$ & $2.9(2)$ & - \\
\hline & 327 & 6.4 & 4 & & & \\
\hline & 436 & $21-25$ & 4 & & & \\
\hline $\mathrm{J} 2317+1439$ & 436 & 13.5 & 3 & $3.94(1)$ & $0.59(4)$ & $0.5(2)$ \\
\hline
\end{tabular}

Notes. Diffractive timescales obtained from the PPTA and NANOGrav datasets. Values with no reference were calculated from the 9-year data set. The first value listed for each MSP is the value used in calculating the structure function. The values $\beta, \sigma_{n}$, and $A$ are produced by fitting the SF and correspond to the stochastic power law exponent, the white noise component, and the periodic amplitude component respectively. Values in parentheses show the uncertainty in the last digit.

${ }^{1}$ Nicastro et al. (2001), ${ }^{2}$ Keith et al. (2013), ${ }^{3}$ Johnston et al. (1998), ${ }^{4}$ You et al. (2007) 
defined as the half-width at $e^{-1}$. This is following the same procedure as described in Levin et al. (2016).

Most observations in the 9-year data set are around 30 minutes long, and for many pulsars, the scintillation timescale is much longer than this integration time. Therefore, it was only possible to measure diffractive timescales in this way for a few of the pulsars in the sample.

Lag bins are equally spaced in log space. Errors on the SF were calculated by combining the propagated errors from the DM time series values and the errors due to uncertainty in the specific realizations in a stochastic process. To constrain those errors, we used simulations of different spectral slopes and different timespans of data, the standard deviation of the SF was calculated for $10^{4}$ realizations at every time lag $\tau$. This was saved as a 3D grid of values (timespan, $\beta, \tau$ ) and then a function was used to interpolate over that grid to give the realization error of the SF value at each $\tau$. We held $\beta$ constant to the value for a Kolmogorov medium as varying the slope had a negligible effect on the derived errors.

\subsection{Results}

\subsubsection{Linear trends and annual periodicities}

Of the $37 \mathrm{MSPs}$ in the data release, 32 show the presence of DM trends, of which five MSPs show piecewise trends over different time spans. With the least squares fitting procedure, we find periods roughly consistent with an annual periodicity \pm 54 days for 17 pulsars; seven of these periods were also detected by the 
Table 2.4 Positions and Corrected Velocities For Three MSPs

\begin{tabular}{lllrr}
\hline \hline PSR & $l$ & $b$ & $\begin{array}{r}v_{E} \\
\mathrm{~km} \mathrm{~s}^{-1}\end{array}$ & $\begin{array}{r}v_{p} \\
\mathrm{~km} \mathrm{~s}^{-1}\end{array}$ \\
\hline $\mathrm{J} 1012+5307$ & $160.35^{\circ}$ & $50.86^{\circ}$ & $-6.1(3)$ & $38(8)$ \\
$\mathrm{J} 1024-0719$ & $251.70^{\circ}$ & $40.52^{\circ}$ & $-15.8(8)$ & $205(30)$ \\
$\mathrm{J} 1738+0333$ & $27.72^{\circ}$ & $17.74^{\circ}$ & $16.8(8)$ & $-25(16)$ \\
$\mathrm{J} 1903+0327$ & $37.34^{\circ}$ & $-1.01^{\circ}$ & $16.0(8)$ & $58(3)$ \\
\hline \hline
\end{tabular}

Notes. Columns are Galactic longitude and latitude of the pulsar, the LOS component of the solar velocity, and the corrected velocity of the pulsar with respect to its local medium. Galactic coordinates were taken from the ATNF Pulsar Catalogue (Manchester et al., 2005, http://www.atnf.csiro.au/people/pulsar/psrcat/).
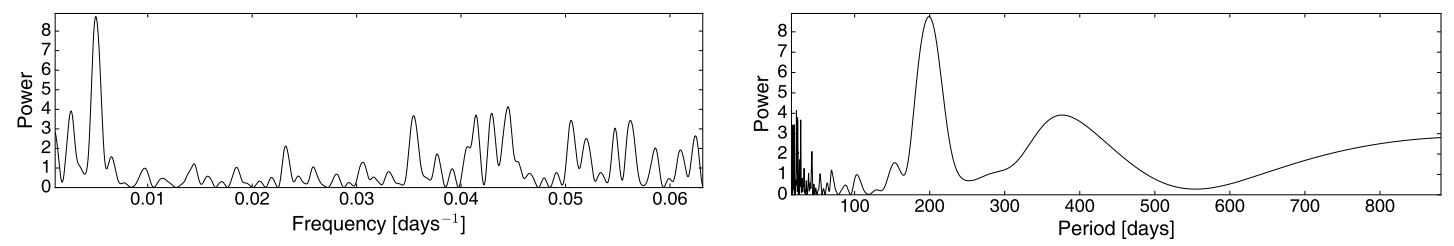

Figure 2.14 Lomb-Scargle periodogram for PSR J0645+5158, shown with frequency (left) as well as period (right). The highest peak occurs at a period of $\sim 200$ days with a false alarm probability of $0.54 \%$, with a secondary peak at an approximately annual period with $\sim 30 \%$ FAP. 
periodogram. The periodogram found significant periods that were not consistent with annual for two pulsars (PSRs J0645+5158 and J1747-4036). A period of 199 days was found for J0645+5158; this roughly semi-annual period could be indicative of several effects in the DM time series (ionosphere, refractive effects from a clump of material along the LOS, or a solar wind event, among other possibilities; Lam et al., 2016). More investigation (and probably more data) would be needed to more definitively identify the source. The power spectrum, seen in Figure 2.14, shows a significant peak at the found period of 199 days and a secondary much less significant annual period, where the uncertainty in both of these periods is equivalent to the cadence of observations. A period of 459 days was found for J1747-4036, however there are less than two years of data for this pulsar so this periodicity may not be robust. Keith et al. (2013) predicted that annual modulations would be seen based on the spectral analyses for PSRs J1024-0719 and J1909-3744, and that they are dominated by the steep linear trend. The authors suggest that a more significant detection could occur through combining data sets. Our trend analysis did not find an annual trend in PSR J1024-0719. We did, however, calculate a period of 366 \pm 5 days for PSR J1909-3744. A linear trend was found in 27 pulsars, 13 of which also exhibit annual trends. Five pulsars exhibit annual trends without a linear trend.

One possible cause of linear trends in $\operatorname{DM}(t)$ is an increasing or decreasing distance between the Earth and the pulsar. For four of the pulsars in the nine-year data set, LOS velocities have been measured with high precision. This allows us to solve for the free electron density around the pulsar. Matthews et al. (2016) calcu-

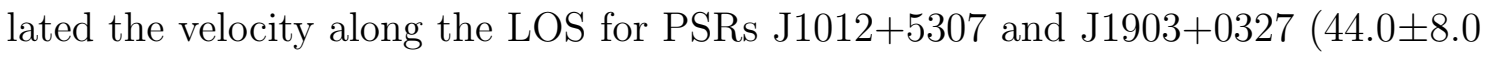


$\mathrm{km} / \mathrm{s}$ and $42.1 \pm 2.50 \mathrm{~km} / \mathrm{s}$ respectively), Kaplan et al. (2016) find a LOS velocity of $221 \pm 30 \mathrm{~km} / \mathrm{s}$ for J1024-0719, and Antoniadis (2013) finds a velocity of $-42 \pm 16$ $\mathrm{km} / \mathrm{s}$ for J1738+0333. We correct these for the Sun's motion by calculating the Suns velocity with respect to the local medium along each of the three lines of sight, given a velocity of $18.0 \pm 0.9 \mathrm{~km} / \mathrm{s}$ in the direction $\mathrm{l}=47.9^{\circ} \pm 3.0^{\circ}$ and $\mathrm{b}=23.8^{\circ} \pm 2.0^{\circ}$ (Frisch et al., 2011). The corrected pulsar velocities are given in Table 2.4. Following Lam et al. (2016), we calculate the corresponding free electron density for each linear trend in DM as

$$
\frac{d \mathrm{DM}}{d t}=n_{e}\left(\mathbf{x}_{p}\right) v_{p}-n_{e}\left(\mathbf{x}_{E}\right) v_{E}
$$

where $n_{e}\left(\mathbf{x}_{p}\right)$ and $n_{e}\left(\mathbf{x}_{E}\right)$ are the free electron densities at the Earth and the pulsar and $v_{p}$ and $v_{E}$ are the LOS velocities of the Earth and the pulsar through their local media. We calculate $n_{e}=2.8 \pm 0.8 \mathrm{~cm}^{-3}$ and $n_{e}=1.9 \pm 0.3 \mathrm{~cm}^{-3}$ for PSRs J1012+5307 and J1024-0719 respectively. These are higher than the typical value for our Galaxy $\left(n_{e} \approx 0.03 \mathrm{~cm}^{-3}\right)$ but local environments can vary drastically, so the linear trends present could be explained by the LOS velocities and could indicate increased ionization as the pulsar is moving away from us. For comparison, Lam et al. (2016) find $n_{e}=7.6 \pm 2.9 \mathrm{~cm}^{-3}$ around PSR J1909-3744 following the same method. For PSR J1738+0333, we find $n_{e}=31 \pm 21 \mathrm{~cm}^{-3}$ which is much higher than a typical value but with high uncertainties given the uncertainties on the pulsar velocity and $d \mathrm{DM} / d t$. For PSR J1903+0327, we calculate a negative $d \mathrm{DM} / d t$, which is not as expected as the pulsar is moving away from us. Using a typical $n_{e}$ we would expect a decreasing linear trend of order $d \mathrm{DM} / d t \approx-7 \times 10^{-7} \mathrm{pc} \mathrm{cm}^{-3} \mathrm{yr}^{-1}$. Therefore, the 
DM variations must be dominated by another effect (i.e. refractive lensing, which may also explain the quasi-periodicities seen for this pulsar; Lam et al., 2016).

Reardon et al. (2016) models the DM variations for 20 PPTA-observed MSPs, nine of which are also included in the NANOGrav 9-year data release (PSRs J0613-0200, J1024-0719, J1600-3053, J1643-1224, J1744-1134, B1855+09, J1909-3744, B1937+21, and J2145-0750). The trends assignments agree between the NANOGrav and PPTA data for PSRs 1024-0719, J1600-3053, J1643-1224, J1713+0747, B1937+21, and J2145-0750. The PPTA data did not show a linear trend for PSR J0613-0200 or periodic trends for PSRs B1855+09 and J1909-3744. Discrepancies could arise due to the difference in data spans, trends that continue or vary after the end of the data set, variations in methods of DM fitting, as well as differences in the trend fitting algorithm; we fit for the period whereas it is fixed at one year by Reardon et al. (2016). There is also the possibility that the DM measured for observed epochs do not agree between the sites due to the difference in observing frequencies used and spatial location; the two data sets may essentially be sampling a different ISM due to multi-path scattering (Cordes et al., 2016).

Lentati et al. (2016) apply a Fourier-based method of DM estimation (see also Lena et al. (2014)) that allows for robust correction of DM variations during epochs without multi-frequency data in the International Pulsar Timing Array (IPTA) data release (Verbiest et al., 2016). The DM noise model used by Lentati et al. (2016) assumes the power spectrum of the variations is in the form of a frequency-dependent power law. It also includes a term that provides power at a one year period; any periodicity found would be in addition to that already included in the model. Lentati 
Table 2.5 Significance of DM peaks for MSPs within $10^{\circ}$ of the ecliptic

\begin{tabular}{llll}
\hline \hline PSR & $\begin{array}{l}\sigma \\
\left(10^{-3} \mathrm{pc} \mathrm{cm}^{-3}\right)\end{array}$ & $\mathrm{DM}_{\text {peak }} / \sigma$ & $\theta$ \\
\hline J0023+0923 & 0.53 & 1.2 & $6.3^{\circ}$ \\
$\mathrm{J} 0030+0451$ & 0.12 & 44.4 & $1.5^{\circ}$ \\
$\mathrm{J} 1614-2230$ & 0.22 & 4.3 & $6.8^{\circ}$ \\
$\mathrm{J} 2010-1323$ & 0.18 & 4.2 & $6.5^{\circ}$ \\
\hline \hline
\end{tabular}

Notes. Columns are the rms $\sigma$ of DM measurements with a Sun-pulsar angle greater than $30^{\circ}$, the ratio of the highest DM value in the data set over the rms, and the minimal angle $\theta$ between the Sun and the pulsar. The rms was calculated only using DM measurements with $\theta>30^{\circ}$ to avoid contamination from the peak DM value. The solar angle corresponds to the minimal angle seen between the pulsar and the Sun in the 9-year data.

et al. (2016) do not find significant annual components in the data set, however this is not surprising as this effect is already being taken into account.

Lentati et al. (2017) find a covariance between temporal variations in scattering and DM. By analyzing PSR J1643-1224, identified here and in the literature as showing periodic variations in DM, Lentati et al. (2017) show that not accounting for high variability in scattering over time where low-frequency (below $1 \mathrm{GHz}$ ) measurements are used to determine $\mathrm{DM}(t)$ introduces periodic structure in the DM time series. Fixing scattering timescales can also result in an underestimation of the uncertainties on $\operatorname{DM}(t)$. This effect is a possible cause of periodic variations in PSR J1643-1224 if the scattering is also variable on roughly annual timescales (Levin et al. (2016) were unable to measure scattering timescale variations for PSR J1643-1224).

The DM is highly anti-correlated with the solar elongation angle for PSRs J0030+0451 and J1614-2230, as can be seen in Table 2.5. There is a moderate correlation in PSRs J0023+0923 and J2010-1323. It is worth noting that in Figure 
2.4.3, the DM measured for the second lowest solar elongation angle is higher than that for the minimum angle; this could be due to a random solar outflow that was not present during subsequent passes near the Sun. Comparison to an inverse square density model for the solar wind is difficult here as the model is an average and hence does not include solar events. The effects of the solar wind are clear in the pulsars with apparent anti-correlation between the peak DM and solar elongation angle. However, because the solar wind can vary stochastically, the overall model may not represent the data fully.

The DM varies by one error bar over a timescale between one month and one year (30 days $<\delta t<365$ days) for 18 MSPs. An additional 10 MSPs had a $\delta t$ approximate to or smaller than the cadence of our observations ( one month). Only four MSPs showed no DM variations beyond measurement error on timescales less than one year, with no measurement calculable for five MSPs due to the absence of a predictable trend to use as a model. This illustrates how quickly the DM can vary by a significant amount and the necessity of observing at $\sim$ week cadences and fitting for DM at every epoch.

The DM trajectory maps (Figures 2.8 through 2.11) are useful in visualizing various ISM features that the pulsar is encountering. A gradient in DM that is in the same direction as the pulsar's motion, as with PSRs J1024-0719 and J1643-1224 for example, could be indicative of an increasing or decreasing distance between Earth and the pulsar or of the pulsar moving through a density gradient. A gradient across the trajectory that is not in line with the pulsar's motion, as with PSRs J06130200 and J1614-2230, cannot arise solely from a change in distance. This coincides 
with the presence of systematic variations; pulsars with linear trends presenting as gradients along the direction of motion and periodic variations due to an overdensity that the pulsar repeatedly probes as it passes through the same LOS annually. Using PSR J0613-0200 as an example, assuming there is a 2D gradient in DM crossing the LOS in front of the pulsar, we find that the gradient has an amplitude of $d \mathrm{DM} / d \tilde{\mathrm{x}} \approx 2.6 \times 10^{-4} \mathrm{pc} \mathrm{cm}^{-3} \mathrm{AU}^{-1}$ roughly perpendicular to the direction of motion on the sky.

\subsubsection{Structure functions}

We have computed SFs for MSPs whose diffractive timescales could be calculated or obtained from the literature, seen in Table 2.3. SFs (see Figures 2.12 and 2.13) could be calculated for 15 of 37 MSPs in the data release. Three MSPs (PSRs J1832-0836, B1953+29, and J2017+0603) whose diffractive timescales were available were omitted because there was fewer than two years of continuous DM measurements. Fit power spectral values can also be seen in Table 2.3. PSRs J0030+0451 and J2145-0750 show fairly flat SFs, which is most likely a result of a white noise dominated data set. Keith et al. (2013) show a SF for J2145-0750 exhibiting similar structure at shorter time lags, but the error bars are too large to allow a detailed comparison.

For J1600-3053, Keith et al. (2013) measure a Kolmogorov SF; You et al. (2007) mention it as being quadratic at shorter time lags becoming less steep at higher lags. Our analysis shows apparent white noise domination for time lags 
below $\sim 100$ days, beyond which the SF is bit more shallow than Kolmogorov for the majority of time lags.

PSRs J0613-0200, J1024-0719, J1643-1224, B1855+09, and J2317+1439 show nearly quadratic power spectra. Our calculated SF for J0613-0200 agrees with You et al. (2007). The SF for J1643-1224 resembles that in Keith et al. (2013). You et al. (2007) show a power law exponent between the expected values for a quadratic and Kolmogorov medium at shorter time lags, with the SF exhibiting a power spectrum below Kolomogorov at higher lags. Our analysis shows a nearly quadratic power law with a distinct turnover present at a time lag of one year before climbing again at higher lags. This could be indicative of an underlying Kolmogorov medium.

You et al. (2007) do not calculate a SF for J1744-1134, but predict it would be Kolmogorov based on previous $d \mathrm{DM} / d t$ measurements. Our calculation compares well to Keith et al. (2013), starting out dominated by white noise then ends roughly Kolmogorov at higher lags. Our analysis finds a power spectrum below Kolmogorov.

PSRs J1614-2230, J2145-0750, J1909-3744, and J1944+0907 are roughly Kolmogorov. PSR J1909-3744 compares well to the calculation in Lam et al. (2016). PSRs J1600-3053, J1713+0747, J1744-1134, and B1937+21 have power-law indices lower than expected for a Kolmogorov medium. PSR J0030+0451 has too large an uncertainty on $\beta$ for a definitive comparison.

The periods found by the SF fitting analysis agree within errors with those found by the DM time series trend analysis for all pulsars except one. A periodic trend was detected in the DM time series PSR B1855+09, but was not found by the SF analysis. 
Here we discuss specific pulsars of interest.

\subsubsection{PSR J1713+0747}

You et al. (2007) show the SF for J1713+0747 as being less steep than a quadratic power law at higher time lags, as does Keith et al. (2013). The SF calculated here looks almost white noise dominated; removing the DM event occurring around MJD 54750 (2008-2009) and re-calculating the SF still yields what looks like a white noise dominated spectrum. We do not believe this is purely white noise because of the correlated structure we see in the time series. However, if that power is evenly distributed over the range of time lags we are concerned with then the SF will appear constant with lag.

Given the time of the observation immediately before the sharp decrease in DM on MJD 54751 and the epoch at which the DM is once again within $1 \sigma$ of the average value, we find an upper limit for the DM event of $\sim 200$ days; using the transverse velocity and distance from Table 2.1 and assuming the structure responsible is located at the pulsar gives an upper limit size to the dispersing region of 4.0 $\pm 0.1 \mathrm{AU}$. With the increased amount of data in the IPTA data set, Lentati et al. (2016) are able to sample the DM event more effectively. They identify the DM event as spanning $\sim 100$ days, with the minimum DM occurring at $\sim$ MJD 54757. 


\subsubsection{PSR B1855+09}

PSR B1855+09 shows a linear SF that does not align with the Kolmogorov or quadratic trendlines when using the diffractive timescale listed in Table 2.3. This could be due to an incorrect or varying diffractive timescale; adding the diffractive timescale as another fitted parameter when fitting the SF gives a value of $\Delta \tau_{D}=$ $55 \pm 1$ minutes, which is more than double the calculated values. The resulting SF is nearly quadratic with some white noise dominating at small time lags.

\subsubsection{PSR B1937+21}

There is an extensive history of SF analysis for B1937+21. Kaspi et al. (1994) found a power law exponent of $\beta=3.874 \pm 0.011$ with a little more than 8 years of data. Cordes et al. (1990) found a similar $\beta$ value that falls between a Kolmogorov and quadratic power spectrum, which agrees with our data at lower lags. Ramachandran et al. (2006) finds a lower value of $\beta=3.66 \pm 0.04$ (compared to our $3.59 \pm 0.01$ ) from 1983 to 2004 that is consistent with a Kolmogorov medium. As with previous studies, Keith et al. (2013) also shows a steady decrease in the DM through the end of 2010, and show a similar Kolmogorov-consistent SF. However, in 2011 the DM started to continuously increase through 2013, which is not a date range that any of the previously calculated SFs covered. It is likely that this latest increase in DM is the reason for the dip at higher lags that are not present in previous data sets. 


\subsection{Discussion}

We examined a large set of DM timeseries from the NANOGrav data set and found evidence on timescales as small as weeks of variations in the vast majority of them. We found evidence of linear trends and annual periodicities, in addition to evidence for discrete ISM structures and one significant non-annual periodicity. We calculated and interpreted SFs for a subset of MSPs and examined DM variations due to LOS motions. These structure functions often appear non-Kolmogorov, but we show that this can be due to the presence of trends in the data and does not necessarily indicate a non-Kolmogorov medium.

DM measurements can inform us about the free electron density along the LOS to a pulsar. In addition, trends due to the changing LOS over time aid in investigating structure in the ISM. Linear trends may be caused by parallel or transverse motion when the free electron density may be changing to a higher or lower than average density in a region. Lam et al. (2016) show that DM variations due to a changing distance between Earth and the pulsar is dominated by parallel motion and that the transverse motion is negligible, entering only as a second order consideration. The free electron density along a particular LOS is typically assumed to be temporally invariant. Examining the scintillation parameters and flux densities of MSPs exhibiting linear DM trends can inform if this is an accurate assumption for that particular LOS. Looking at the LOS velocities for four pulsars, we expect that three of them (PSRs J1012+5307, J1024-0719, and J1738+0333) are consis-

tent with exhibiting linear trends due to the pulsar's increasing distance. The DM 
variation amplitude is much larger than expected given the average Galactic free electron density, indicating that the pulsars may be in overdense regions or ionize the materials along their paths (Chatterjee \& Cordes, 2004). The velocity and direction of motion of PSR J1903+0327 suggest that its increasing distance from the Earth cannot explain its trend. Annual variations may be due to a variety of solar effects and their amplitude is influenced by the relative velocity of the MSP when compared to the Earth's orbital motion as well as the Sun's velocity as it moves through the Galaxy. We see specific cases where the solar wind is the dominant factor in producing annual variations. For others, variations could be due to a cloud or gradient along the LOS. Scintillation parameters and fluxes could indicate whether variations were due to clumps, refraction, scattering variability, or local increases in electron density (such as solar wind) (Stinebring \& Condon, 1990; Clegg et al., 1998; Stinebring et al., 2000; Lentati et al., 2017). Future work may result in some determination, particularly if scintillation parameters over time are available and incorporated.

The relationship between linear and periodic variations and the direction of a gradient along the LOS can also be seen visualized by DM trajectory maps. Five MSPs show only annual trends and 14 show only linear trends, while 13 exhibit both trends. More than half of the MSPs showed significant DM variation beyond our measurement error over the timescale of one month to one year. There are 10 MSPs with timescales less than 31 days, which is on par with the average cadence of our observations. Of those, four MSPs have timescales of 14 days or less, which is the size of the fitting window used for DMX in the 9-year data release. It is therefore 
imperative that we fit for DM at every epoch due to the scale of the variations over these timescales, as well as observe as often as possible to minimize the time between DM measurements due to the rapid variation seen in some MSPs.

For three PSRs, the SFs appear to be dominated by white noise, resulting in a flat power spectrum. Three MSPs have very nearly quadratic power spectra, with two (PSRs J1024-0719 and J2317+1439) having a $\beta$ value within 1\% of quadratic. Lam et al. (2016) suggest discrete structures in the ISM as well as the changing distance will contaminate the SF resulting in a quadratic power spectrum. This steeper than Kolmogorov value could be indicative that the time lag is smaller than the crossing time for the structure probed during the time series. Higher values than consistent with a Kolmogorov medium could be attributed to present trends or systematic variations in addition to a Kolmogorov medium, particularly with the previously discussed difficulties in disentangling a linear trend component from a stochastic one. We cannot impose priors without assuming something about the contributions from the ISM that we are trying to constrain, and which may not actually be the case. There are also more possible sources of error in the calculation of the $\beta$ values than we have included. We have accounted for the random and stochastic uncertainties but not systematic uncertainties, which can result from variability of white noise statistics over time from changing backends, the variation in the diffractive timescale, and the fact that the models used here could be incomplete in describing the ISM. Therefore, while the $\beta$ values presented here are illustrative, their errors bars are likely under-estimated. In addition, care should be taken when using these values to make inferences about the ISM due to possible covariances and 
systematics present.

\subsection{Acknowledgments}

The NANOGrav project received support from the National Science Foundation (NSF) PIRE program award number 0968296 and NSF Physics Frontier Center award number 1430284. MLJ acknowledges support from West Virginia University through the STEM Mountains of Excellence Fellowship. Data for the project were collected using the facilities of the NRAO and the Arecibo Observatory. The NRAO is a facility of the NSF operated under cooperative agreement by Associated Universities, Inc. The Arecibo Observatory is operated by SRI International under a cooperative agreement with the NSF (AST-1100968), and in alliance with the Ana G. Méndez-Universidad Metropolitana, and the Universities Space Research Association. 


\section{Chapter 3}

\section{Investigating the Candidate Displaced Active Galactic Nucleus in}

\section{NGC 3115}

\subsection{Abstract}

The nearby galaxy NGC 3115 contains a known radio-emitting, low-luminosity active galactic nucleus (AGN), and was recently claimed to host a candidate AGN displaced $14.3 \mathrm{pc}$ from the galaxy's optical photocenter. Our goal is to understand whether this represents a single offset AGN, an AGN in orbit around a central black hole, or something else. We present a new, sensitive $\left(\mathrm{RMS}=4.5 \mu \mathrm{Jy}\right.$ beam $\left.^{-1}\right)$ $10 \mathrm{GHz}$ image, which finds evidence for only one AGN. We place a stringent limit on the radio luminosity of any secondary supermassive black hole of $L_{10 \mathrm{GHz}}<5.6 \times 10^{33}$ ergs/s. An analysis of the relative positioning of the radio core, X-ray nucleus, and stellar bulge in this galaxy indicate that the radio source is centrally located, and not offset from the galactic bulge. This provides an argument against a single offset AGN in NGC 3115, however does not provide conclusive evidence against the purported offset AGN as an in-spiralling secondary black hole.

Submitted to ApJ.

Contributing authors: Sarah Burke-Spolaor, Kristina Nyland, Joan M. Wrobel 


\subsection{Introduction}

Supermassive black hole (SMBH) binaries should form during major galaxy mergers. Through the loss of orbital energy, the SMBH pair will eventually coalesce, releasing an enormous of amount of energy in the form of gravitational waves. Gravitational waves produced by SMBH mergers would be detectable by pulsar timing arrays (e.g. Arzoumanian et al., 2018b). Asymmetric gravitational wave emission can produce a strong force - or "kick" - to the final SMBH. If this kick is smaller than the host escape velocity, it can induce oscillation of the SMBH about the galaxy's core (kick velocities can be up to several thousand $\mathrm{km} / \mathrm{s}$, causing the SMBH to be ejected from the galaxy; Blecha et al., 2013). The SMBH should eventually settle into the host galaxy's center due to drag and other dynamical interactions with the stellar and gas environment (e.g. Begelman et al., 1980; Campanelli et al., 2007). While recoiling SMBHs may return to the center after several Gyr, SMBH recoils in gas-poor galaxy mergers can remain non-centrally located for much longer periods of time (Blecha et al., 2013).

SMBH recoils induced by these kicks have astrophysical implications for the host galaxy, such as SMBH and galaxy evolution, galactic core structures, galaxySMBH scaling relations, and the dependence of gravitational wave signals on redshift, among others (Komossa, 2012). The identification of potential recoiling SMBHs is therefore important in exploring past galaxy mergers, information on kick properties, as well as investigating predictions made via numerical relativity.

There are not many small-orbit binary SMBH systems known, with only a 
few examples below separations of $1 \mathrm{kpc}$ (e.g. Rodriguez et al., 2006). There have likewise been scant discoveries of post-merger systems where the SMBH is seen in a state of offset/recoil, with only a few unconfirmed candidate systems (Komossa et al., 2008; Postman et al., 2012; Blecha et al., 2013; Lena et al., 2014; Chiaberge et al., 2017). To achieve sufficient AGN offsets such that the object is identifiable as an offset system, a large kick velocity is necessary. High kick velocities, while possible, are likely to cause stripping of much of the emissive material from the SMBH after its departure from the galactic center; therefore bright AGN with large offsets are likely to be rare.

At a distance of $10.2 \mathrm{Mpc}$, NGC 3115 is the nearest host of a billion-solarmass black hole, and represents one of the first SMBHs with an accurate mass measurement based on stellar or gas dynamics $\left(\mathrm{M}_{\mathrm{BH}}=9.6 \times 10^{8} M_{\odot}\right)$ (Kormendy \& Richstone, 1992; Gültekin et al., 2009). At the object's distance 1" $=49.5$ pc, making the task of spatially resolving any offset more feasible than for more distant sources. Compact radio emission with a luminosity of $3.1 \times 10^{35} \mathrm{ergs} / \mathrm{s}$ that is coincident with the optical center in the nucleus of NGC 3115 was first detected by Wrobel \& Nyland (2012) by analyzing archival VLA data, with an Eddington luminosity of $\mathrm{L}_{\mathrm{Edd}}=1.2 \times 10^{47} \mathrm{ergs} / \mathrm{s}$. This detection is also coincident with an Xray candidate nucleus identified by Wong et al. (2011), who conservatively estimate the luminosity as $\mathrm{L}_{X}<4.3 \times 10^{38} \mathrm{ergs} / \mathrm{s}$. These data suggest the existence of a low-luminosity active galactic nucleus (AGN) residing in the center of this galaxy.

Using the Gemini-South telescope, Menezes et al. (2014) reported the detection of a broad-line $\mathrm{H} \alpha$ emission with a luminosity of $\mathrm{L}_{\mathrm{H} \alpha}=4.2 \pm 0.4 \times 10^{37}$ ergs 
$\mathrm{s}^{-1}$ that was displaced from the photometric center of NGC 3115's stellar bulge by $290 \pm 50$ mas $(14.3 \pm 2.5 \mathrm{pc})$. Upon inspecting several possibilities including a rotating relativistic disk around the central black hole and imprecise starlight subtraction, they concluded that the emission is most likely associated with an offset AGN. If this detection genuinely represents an offset AGN, there are two potential interpretations. First, it is possible the black hole fueling the offset AGN is actually in a pc-scale binary with a second black hole situated at the photocenter. Alternately, the AGN displacement could be the result of a black hole that has been kicked from the galaxy photocenter via recoil.

In this paper we present a radio search for evidence of a binary or offset AGN in NGC 3115. Section 4.3 reports new $10 \mathrm{GHz}$ data collected with National Science Foundation's Karl G. Jansky Very Large Array (VLA), Section 3.4 discusses the new radio measurements in the context of our detection of only one radio core, and reports a closer examination of the relative positions and astrometric errors of several different measurements of the AGN and galaxy center. We discuss the implication of these results in Section 3.5.

\subsection{Very Large Array Data}

We observed NGC 3115 with the VLA at X-band in the A-configuration on 12 June 2015, with 84 minutes on-target. The observational set-up had 64 frequency channels in each of 32 unique spectral windows, across the range $7.976 \mathrm{GHz}$ to $12.024 \mathrm{GHz}$ with a center frequency of $\sim 10 \mathrm{GHz}$. The target pointing position 
was 10:05:13.927, -07:43:06.96. We performed primary flux density and bandpass calibration using standard VLA calibrator 3C286, and used J1007-0207 as a phase calibrator.

We calibrated the data using the VLA calibration pipeline, and interactively deconvolved the images using the CLEAN algorithm in the CASA software package. The RMS of our final image is $4.5 \mu \mathrm{Jy}_{\text {beam }^{-1}}$. The synthesized beam had major and minor axes of 340 mas and 150 mas respectively, with a position angle of $38.34^{\circ}$. Multi-frequency synthesis was performed to account for the large fractional bandwidth with nterms $=1$. We used briggs weighting with a robust value of 0.0 and a minpb of 0.2 . The cellsize was set to 36 mas.

We also made a wide-field image to look for other objects in the field unrelated to this work following the same procedure discussed above; this wide-field image was not used in our analyses but was used to make Figure 3.2, hence the different beam sizes listed for Figures 3.1 and 3.2.

\subsection{Analysis of Available Data}

\subsection{1 $10 \mathrm{GHz}$ Measurement Results}

Our new radio image improved on the RMS sensitivity of our previous image by a factor of $\sim 5$. Figure 3.1 shows a compact source located in the center of NGC 3115 down to our detection limit of three times the RMS. The IMFIT procedure in CASA was used to fit a two-dimensional elliptical-Gaussian to this sole source, yielding the integrated flux density, position, and one-dimensional position error appearing in 


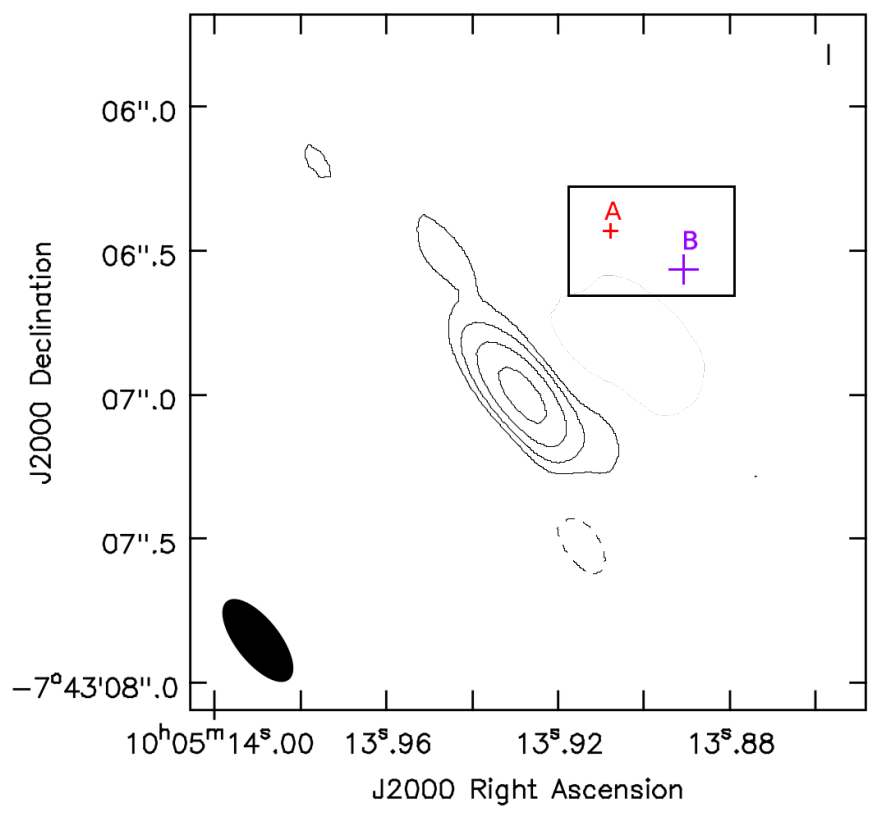

Figure 3.1 Contours of the 10-GHz emission from NGC 3115 at -3, 3, 6, 12, and 24 times the RMS level in the image detected with the VLA. The beam is displayed in the bottom left corner, with major and minor axes of 340 mas and 150 mas respectively and a position angle of $38.34^{\circ}$. The noise level is $4.5 \mu \mathrm{Jy}_{\mathrm{beam}}{ }^{-1}$. The relative positioning of the kinematic center (labelled "B") and the purported offset AGN (labelled "A") of Menezes et al. (2014) are represented by the purple and red crosses, respectively. Note that these are not absolute positions; they are displayed here to demonstrate that we should have been sensitive to two AGN if both were radio-emitting at the relative separation reported by Menezes et al. (2014). The error bars on these two points represent the reported positional error for the bulge, and the AGN offset error for the AGN. 
Table 1. The tabulated position errors are reported as the radius of the error circle at the $95 \%$ confidence level. The flux density error is the quadratic sum of the $3 \%$ scale error (Perley \& Butler, 2013) and the fit residual. The position error is the quadratic sum of terms due to the phase-calibrator position error (less than 2 mas), the phase-referencing strategies (estimated to be 100 mas), and the signal-to-noise ratio of the component (4 mas). The source was found to be point-like, with upper limits of 200 mas $(9.9 \mathrm{pc})$ on its major axis and 60 mas $(3.0 \mathrm{pc})$ on its minor axis, for a position angle of $39.3 \pm 2.2$ degrees.

We detected two additional 10-GHz sources, each offset by more than $1.5^{\prime}$ from the nuclear $10-\mathrm{GHz}$ source and thus unlikely to be associated with it. As an independent cross-check of the VLA position error for the nuclear 10-GHz source (Table 1), we searched the literature for counterparts to the two offset sources. Only one had any counterparts. Figure 3 shows that offset 10-GHz source plus the positions of its X-ray and ugi counterparts (Lin et al., 2015; Cantiello et al., 2015, 2018), and serves to validate the VLA astrometry.

Using previous radio data as listed in Table 3.1 along with the data presented here, for the NGC 3115 nuclear source we measure a spectral index of $\alpha=-0.34 \pm$ 0.09. This is consistent with the index measured by Wrobel \& Nyland (2012) of $\alpha=-0.23 \pm 0.20$ (Fig. 3.3). The relatively flat spectral index of the emission indicates that this emission is likely related to a radio core component, i.e. marks the location of a SMBH rather than marking a distant jet outflow. The flat spectrum and persistence of the source show that it is likely in a low-hard or quiescent state, consistent with the SMBH accreting slowly from the hot gas traced by the X-rays. 


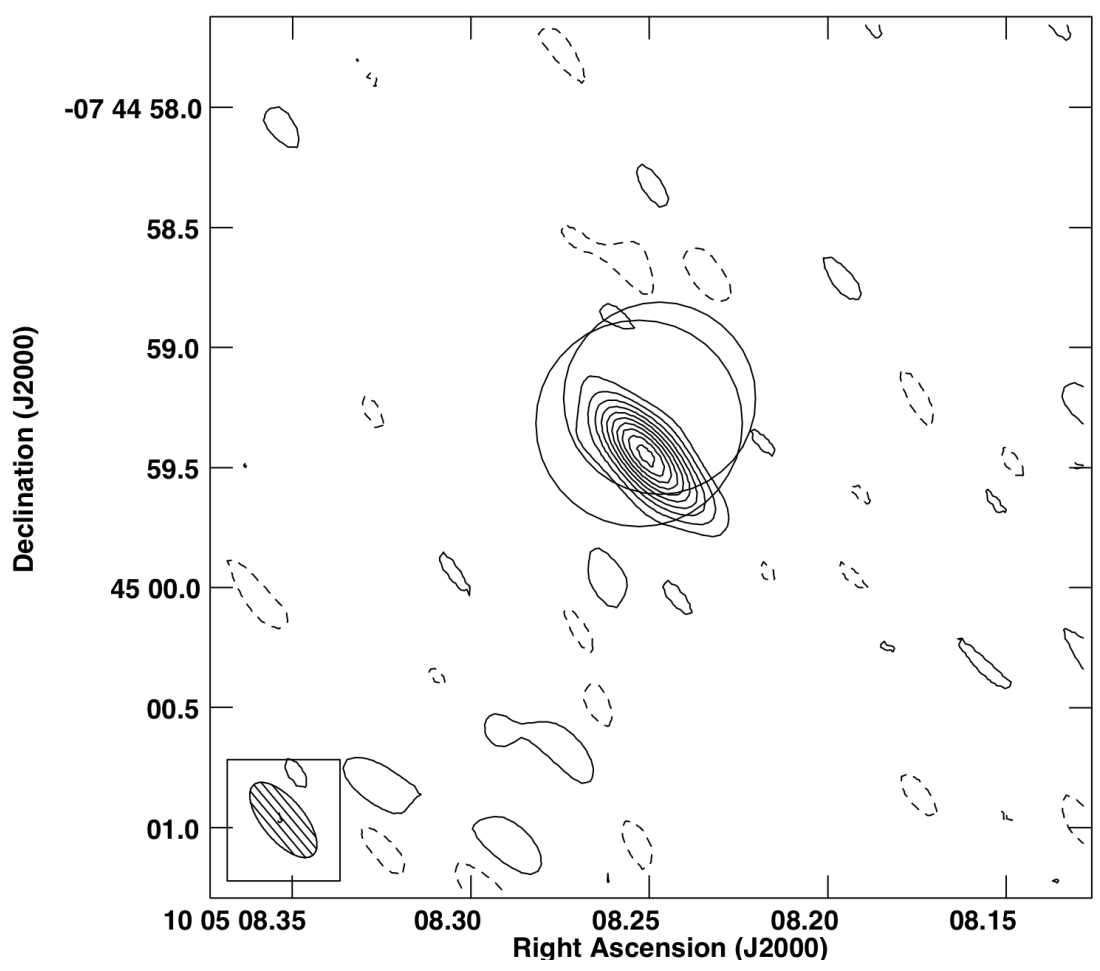

Figure 3.2 VLA image of Stokes $I$ emission near $10 \mathrm{GHz}$ centered on a background source with an X-ray counterpart (Lin et al., 2015) and an ugi counterpart (Cantiello et al., 2015, 2018). The symbols encode the counterpart positions and their errors at the $95 \%$ confidence level. For the $10-\mathrm{GHz}$ image, the local rms noise is 11.4 $\mu \mathrm{Jy}_{\text {beam }^{-1}}(1 \sigma)$ and the beam dimensions at FWHM are 382 mas times 173 mas with a position angle of $40^{\circ}$ (hatched ellipse). The allowed contours are at $1 \sigma$ times $-6,-4,-2,2,4,6,8,10,12,14,16,18$, and 20. Negative contours are dashed and positive ones are solid. 


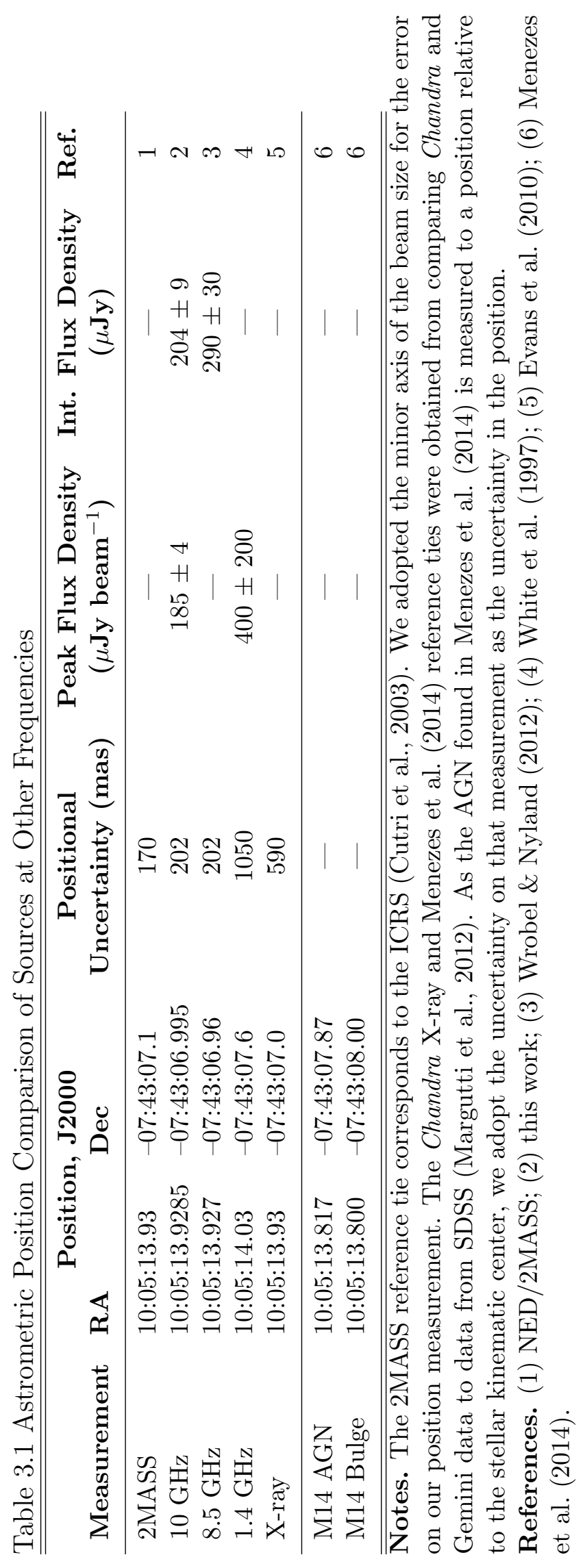




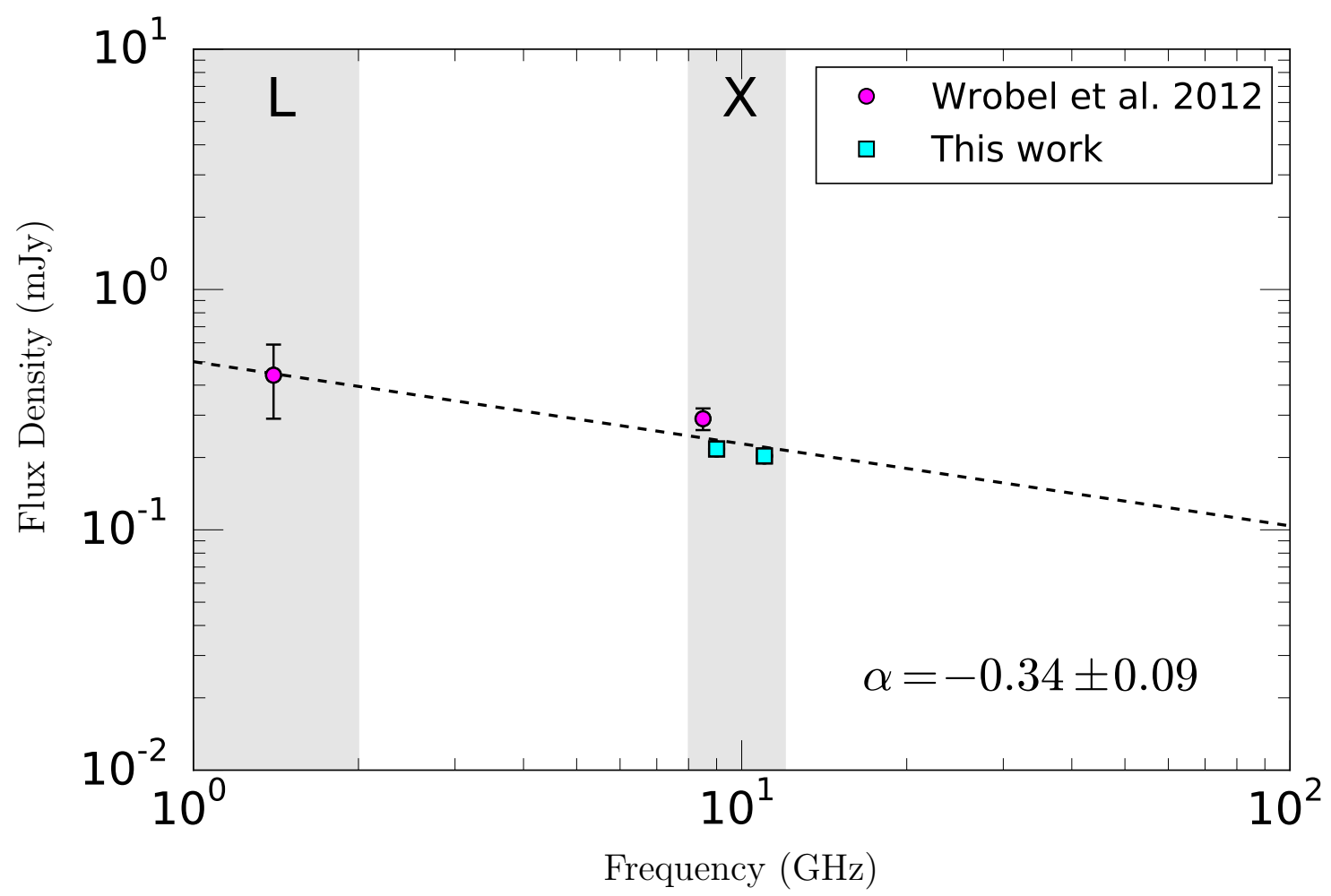

Figure 3.3 Flux density with frequency. Magenta circles represent data at 1.5 and 8.5 GHz from White et al. (1997) and Wrobel \& Nyland (2012), respectively, while the cyan squares signify data from this work, measuring the flux density after splitting the data into two sub-bands centered on 9 and $11 \mathrm{GHz}$.

The integrated flux measured in our new broadband VLA data from $8-12 \mathrm{GHz}$ is consistent with that from archival VLA observations at 8.5 GHz (Table 3.1); thus, in the absence of strong variability, there is no evidence for significant deviation from a single power-law in this radio component (i.e. we are not clearly observing a self-absorption turn-over, nor do we seem to be seeing two distinct regions with vastly different properties within our beam).

It is clear that we have not detected any radio source related to NGC 3115 except for the one previously reported by Wrobel \& Nyland (2012). We initially set out to test the report of Menezes et al. that there was an active nucleus offset from 
the kinematic and photometric center of NGC 3115. As hypothesized in their paper, this could mean they either detected a single offset black hole, or an inspiralling black hole offset from another at the galaxy center, or that there is simply a single black hole at the galaxy center and the offset emission detected by Menezes et al. is caused by, for instance, an outflow. However, several questions remain in the investigation of our initial hypotheses: First, should we have detected a secondary black hole, given the detection of the first one? Second, can we determine whether this object corresponds to the galaxy photocenter or to the purported offset AGN of Menezes et al. (2014)? Third, might the central radio source actually encompass two SMBHs? The first question we address here, and the latter two require a discussion of the relative astrometry of measurements in other wavebands; this is discussed in Section 3.4.3.

\subsubsection{Would we have detected a distinct SMBH companion?}

The radio nucleus in this system has a low luminosity of $L_{10 \mathrm{GHz}}=8 \times 10^{34}$ ergs/s. In considering the hypothesis that this target could contain a binary SMBH, we want to assess the probability that we should have detected a secondary SMBH in our observation given our limiting flux of three times the RMS of the off-source image, $S_{\text {lim }}=13.2 \mu \mathrm{Jy}_{\text {beam }}{ }^{-1}$. At the distance of NGC 3115 this corresponds to a limiting luminosity of $L_{\mathrm{lim}}=5.6 \times 10^{33} \mathrm{ergs} / \mathrm{s}$. Due to the excellent VLA sensitivity and the small distance of this source, our limit on the luminosity of any secondary AGN is exceptionally low; in fact it lies several order of magnitudes below the span 
of published radio-quiet quasar distributions. If there is a companion, we would classify any secondary SMBH in this system as "radio-silent" (Padovani et al., 2015; Padovani, 2016).

Past work has assessed the probability of finding a secondary AGN by integrating over radio luminosity functions down the limiting luminosity in an observation (e.g. Burke-Spolaor, 2011). If this target had a radio-loud or even radio-quiet secondary SMBH by the standard definitions, we should have detected it. It is possible that a secondary SMBH is not in an active state at all, in which case no waveband would have detected its emission. Lützgendorf et al. (2016) discuss gas patchiness as a result of stellar winds; the presence of a second SMBH accreting rapidly from a gas patch is also possible, which would show up as a broad-line AGN and only rarely exhibit radio emission.

\subsubsection{Multi-wavelength astrometry}

Understanding the relative positions of the radio and X-ray emission, the purported offset AGN of Menezes et al. (2014), and the galaxy kinematic center and/or photocenter is key to our interpretation of this object. The study of Menezes et al. (2014) benefited from precise position comparisons due to analysis of relative positioning within a single observation, while our study is by nature limited by astrometric and measurement errors. We will both examine astrometric errors, and re-examine the results of Menezes et al.

While NGC 3115 is a well-studied object, there are relatively few works re- 


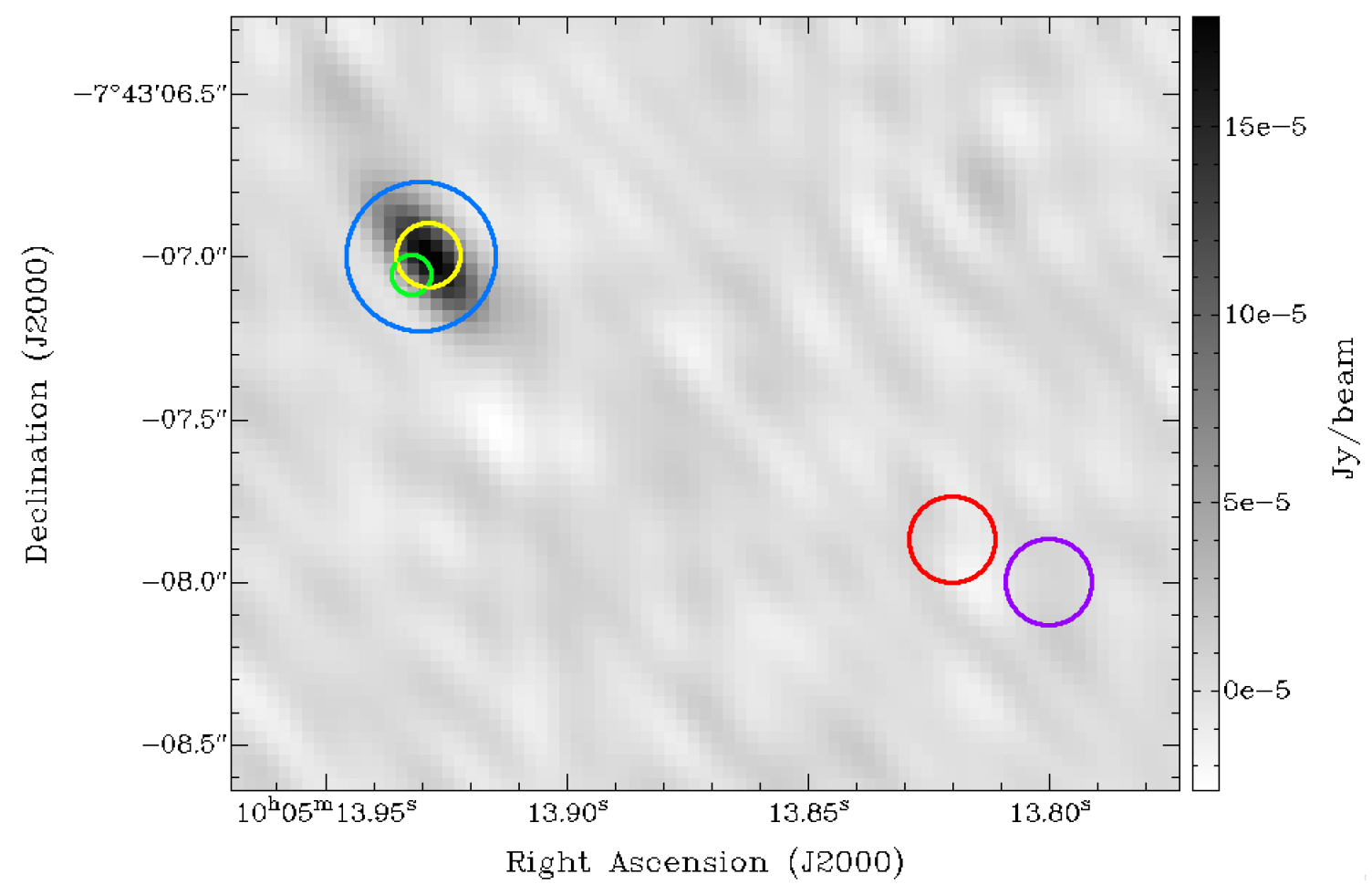

Figure 3.4 The relative positions of the data listed in Table 3.1, with our $10 \mathrm{GHz}$ radio image shown in greyscale. The circle sizes represent the quadrature sum of the astrometric and measurement errors for each data point. The data are as follows: $2 \mu \mathrm{m}$ 2MASS centroid (green); X-ray core (blue); our radio position (yellow). The kinematic center measured by Menezes et al. (2014) is shown in purple (Menezes, private comm.). Their reported offset AGN is shown in red, and is positioned in reference to the purple circle's position. 
porting on the actual position of the galaxy's kinematic center. Here we assess the optical photocenter, the X-ray component, and the radio component to understand what confidence we can have in their relative positioning. Unfortunately, there were insufficient numbers of objects detected both in our image and the X-ray/2MASS data to do a direct astrometric comparison. Instead, the reference tie and measurement errors reported by source publications for each observation are identified in Table 3.1.

The $8.5 \mathrm{GHz}$ observations were also performed with the VLA with the same frame tie accuracy (Wrobel \& Nyland, 2012), while the $1.4 \mathrm{GHz}$ measurement comes from the FIRST survey, which reports a mean astrometric precision of 50 mas (White et al., 1997). The $2 \mu \mathrm{m}$ photocenter position for NGC 3115 was taken from $2 \mathrm{MASS}$. The 2MASS survey is tied to the Tycho 2 catalog, which is accurate to $\sim 70$ mas (Cutri et al., 2003). The position and error listed is that for the NGC 3115 photocenter from the 2MASS point source catalog (Skrutskie et al., 2006). The Chandra X-ray nuclear component was obtained from the Chandra Source Catalog (Evans et al., 2010). The localization of any potential AGN-related nucleus is limited by the fact that there is no nuclear point source, only a plateau of X-ray emission in the core of this galaxy (Wong et al., 2011). The X-ray reference tie is as quoted by (Evans et al., 2010) for the $1 \sigma$ external astrometric error. While the absolute astrometry of Gemini (used by Menezes et al. 2014) is about as accurate as that of Chandra, the astrometrically corrected position information was not reported by Menezes et al. (2014). The AGN and bulge reported by Menezes et al. (2014) are relative positions in the frame of their observation, thus we do not show these errors 
in Fig. 3.4.

Menezes et al. (2014) fit the 2D kinematic profiles of the data in order to obtain a position measurement for the offset broad $\mathrm{H} \alpha$ emission line that indicates the presence of an AGN. They compare this position to the kinematic center of the galaxy (as determined by the velocity dispersions in that region) as well as the stellar bulge center (they equate the image of their collapsed data cube as representing the center of the stellar bulge) and determine that the AGN is coincident with neither the kinematic nor stellar bulge centers of NGC 3115.

In their analysis, Menezes et al. identified an offset of $\sim 290 \pm 50$ mas $(\sim 14.3 \pm 2.5$ pc), with the uncertainties determined using a Monte Carlo simulation. They determine that the kinematic center is coincident with the stellar bulge center, however do not report an absolute position of either the kinematic center, bulge center, or offset AGN. Based on observation headers that are not likely astrometrically corrected, the Gemini pointing center (directed at the stellar bulge center) was at a position of J2000 RA, Dec 10:05:13.80, -07:43:08.00 (R. Menezes, private comm.). The position of the off-centered AGN is reported as $\sim 260$ mas east and 130 mas north from the stellar bulge center, giving an uncorrected AGN position of J2000 RA, Dec 10:05:13.82, -07:43:07.87.

The relative positions and net position errors are displayed atop 2MASS contours in Figure 3.4. The AGN detected by (Menezes et al., 2014) is offset 1.84" from our detected radio source, and their position measurement of the stellar kinematic center is $2.89^{\prime \prime}$ from the 2MASS photocenter; thus there are clear residual absolute astrometry offsets in the observations of Menezes et al. (2014). 


\subsection{Discussion and Conclusions}

\subsubsection{Where is the radio AGN in NGC 3115?}

As shown in Figure 3.4, the position of our radio AGN agrees with the stellar bulge center as indicated by 2MASS, to significantly less than the separation between the offset between the bulge and AGN measured by Menezes et al. As such, it appears that the black hole related to this radio AGN is likely resident in, and not offset from, the galaxy center. As seen in Figure 3.4, the position listed in the Menezes et al. FITs data file header do not appear to have any absolute astrometric correction applied. Regardless, if the bulge center were shifted to the 2MASS position, it is clear that the radio AGN is not colocated with the purported offset AGN.

\subsubsection{Does NGC 3115 contain a binary, offset, or singular central AGN?}

Based on the relatively flat spectrum of the radio AGN and its colocation with the stellar bulge, it is clear that NGC 3115 does not contain a singular offset (recoiling or wandering) AGN. It is possible that the position-offset $\mathrm{H} \alpha$ line does represent a separate $\mathrm{SMBH}$, in which case the offset system may still be undergoing

inspiral after a previous merger. Assuming this SMBH has a mass $\gtrsim 10^{8} M_{\odot}$, the dynamical friction timescale of such an object at this separation is on the order of $100 \mathrm{kyr}$, thus relatively short but not so infeasible to have detected it at this state 
of inspiral (Lacey \& Cole, 1993). The 2D kinematic measurements of Menezes et al. demonstrated some support for this argument, indicating that the isocontours of velocity dispersion demonstrated an elongated, elliptical shape, rather than showing a singular peak at the photometric center. However, the elongation was not along the axis of the AGN offset, suggesting some ongoing mobility of this secondary SMBH in the system under this hypothesis. There are no other large-scale indications that NGC 3115 underwent a merger in the last few Gyr. Finally, it is possible that NGC 3115 simply contains a single, centrally located low-luminosity AGN that is giving rise to the radio source and some component of the central X-ray emission. If the offset source is indeed its own AGN, it appears to have no associated radio emission.

\subsection{Acknowledgments}

MLJ and SBS are members of the NANOGrav Physics Frontiers Center which is supported by NSF award 1430284. SBS is supported by NSF EPSCoR award number 1458952. We thank R. B. Menezes for providing helpful clarifications about the Gemini observations we discuss in this report. The National Radio Astronomy Observatory is a facility of the National Science Foundation operated under cooperative agreement by Associated Universities, Inc. 


\title{
Chapter 4
}

\section{Evaluating Low Frequency Observations at the GMRT in}

\author{
Combination with NANOGrav Data
}

\subsection{Abstract}

The North American Nanohertz Observatory for Gravitational Waves (NANOGrav)

project has the primary goal of detecting and characterizing gravitational waves through high precision pulsar timing. The characterization of interstellar effects is important in order to achieve the precision necessary for gravitational wave detection. Ionization in the interstellar medium causes frequency-dependent time delays due to effects like dispersion and scattering. The lower frequency coverage as well as simultaneous dual-frequency observations available at the Giant Metrewave Radio Telescope (GMRT) have the potential to provide better dispersion measurement than the data taken by NANOGrav. We present GMRT timing data for 10 millisecond pulsars observed at 322 and $607 \mathrm{MHz}$, and compare the dispersion measure estimates obtained with those obtained through NANOGrav observations with the Arecibo Observatory. We analyze the predicted effect of incorporating this low

In prep

Contributing authors: Maura A. McLaughlin, Jayanta Roy, Michael T. Lam, James M. Cordes, Bhaswati Bhattacharya, and the NANOGrav IMM and Timing groups 
frequency data on DM uncertainties. We discuss discrepancies between DMs and investigate possible astrophysical sources of variations in DM measurements. We discuss the possible advantages of incorporating GMRT data into timing efforts for understanding and mitigating interstellar effects as well as improving timing precision.

\subsection{Introduction}

There are currently three global pulsar timing array (PTA) efforts: the North American Nanohertz Observatory for Gravitational waves (Arzoumanian et al., 2018b), the European Pulsar Timing Array (EPTA; Desvignes et al., 2016), and the Parkes Pulsar Timing Array (PPTA; Reardon et al., 2016). These three experiments make up the International Pulsar Timing Array (IPTA; Verbiest et al., 2016), whose primary goal is the detection of nanohertz frequency gravitational waves (GWs) using pulsar timing. The NANOGrav 11-year data set, the most recent data release from the collaboration, includes 45 millisecond pulsars (MSPs; Arzoumanian et al., 2018b). The data set includes up to 11 years of data for each MSP taken at the Arecibo Observatory and the Green Bank Observatory at frequencies between 327 $\mathrm{MHz}$ and $2.3 \mathrm{GHz}$.

Timing models are constructed by accounting for all known effects on the pulsar times of arrival (TOAs) in order to minimize the differences between the measured and model predicted TOAs (i.e. timing residuals). The detection of GWs with pulsar timing requires TOA accuracy less than $\sim$ microsecond. In most 
cases, time delays produced by interstellar effects surpass the MSP pulse periods and therefore must be accounted for in precision timing (e.g. Demorest et al., 2013; Arzoumanian et al., 2015).

One of these such effects is dispersion. As the radio pulse travels through the interstellar medium (ISM), it encounters ionized plasma along the way. The DM is the integrated column density of free electrons along the line of sight (LOS) to a pulsar

$$
\mathrm{DM}=\int_{0}^{d} n_{e}(l) d l
$$

where $n_{e}$ is the free electron density along the LOS and $d$ is the distance. DM therefore can be used to infer the distance to the pulsar by assuming a free electron density model for the Galaxy (e.g. NE2001, Cordes \& Lazio, 2002). The time delay due to dispersion scales as $t \propto \mathrm{DM} / \nu^{2}$ where $\nu$ is the observing frequency; DM can be solved for by observing at multiple frequencies and comparing the respective time delays. Due to the changing LOS, DM is not constant in time (i.e. DM is actually $\mathrm{DM}(t))$; these DM variations affect the timing and must be monitored and corrected for in the data. DM variations can arise for many reasons, including a changing distance between the Earth and the pulsar, varying solar wind, and ionospheric effects. DM variations can also exhibit structure in the form of periodicities and trends.

Trends due to the time-variant LOS are useful in investigating structure in the ISM. Linear trends may be caused by parallel or transverse motion when the free electron density may be varying to higher or lower values than the average 
density in a region. Lam et al. (2016) show that DM variations due to a changing distance between the Earth and the pulsar is dominated by parallel motion and that the transverse motion is negligible, becoming relevant only as a second order consideration. The free electron density along a particular LOS is generally assumed to be time invariant; the examination of scintillation parameters and flux densities of MSPs that exhibit linear DM trends can inform if this assumption is accurate given a particular LOS.

Annual variations may be caused by various solar effects; their amplitude is determined by the relative velocity of the MSP in comparison to the orbital motion of the Earth as well as the solar velocity through the Galaxy. In addition to solar effects, annual variations may also be caused by a gradient along the LOS. As with linear DMs, scintillation parameters and fluxes could inform whether variations were caused by clumps, refraction, scattering variability, or local increases in electron density (e.g. solar wind) (Stinebring \& Condon, 1990; Clegg et al., 1998; Stinebring et al., 2000; Lentati et al., 2017). A more thorough analysis of DM trends seen in the NANOGrav 9-year data set (Arzoumanian et al., 2015) can be seen in Jones et al. (2017).

Turbulence in the ISM which causes trends is typically described as having a Kolmogorov power spectrum, meaning we expect to find larger variations over longer timescales. The power spectrum used to describe a Kolmogorov ISM is

$$
P(q) \propto q^{-\beta}, q_{\text {outer }} \leq q \leq q_{\text {inner }}
$$


where $q$ is the reciprocal of the size scale, and $\beta$ is the power spectrum exponent. A Kolmogorov medium corresponds to $\beta=11 / 3$, while the highest value expected for turbulence in the ISM (for an inner scale shorter than $10^{9} \mathrm{~m}$ ) is $\beta=4$ (Rickett, 1990). The outer scale is described as the size at which the ISM is no longer homogeneous whereas the inner scale is the point where dissipation occurs in the material along the line of sight.

It is difficult to distinguish between DM trends or describe the ISM as showing a specific component. As stated above, a linear trend in DM could be due to an increasing distance between the Earth and the pulsar; however, a purely stochastic medium would also present as a monotonic increase in $\operatorname{DM}(t)$. There is substantial evidence of contamination between the stochastic and trend components. In many cases, a linear trend fit over the DM timeseries will absorb part of the stochastic component. Knowledge of some prior about the contributions from the ISM would need to be known in order to disentangle a linear trend component from a stochastic one. The presence of DM trends or systematic variations in addition to a Kolmogorov medium will also appear as a steeper power law. Jones et al. (2017) discuss in depth the covariances between DM trends, periodicities, noise, and a stochastic medium.

A second effect is scattering. As the pulse travels through the ISM, it will be scattered due to inhomogeneities in the ISM which cause multi-path propagation of the pulse. These multiple ray paths introduce a delay in the TOA. Like DM, scattering delays are also time variable due to dynamic processes in the Galaxy. Scattering scales as $\nu^{-4}$ and is therefore much more influential at lower observing frequencies. Pulse scattering becomes particularly pertinent for MSPs at high DMs (Bhat et al., 
2004). Scattering cannot be corrected as easily as DM, but can be partially corrected using high resolution dynamic spectra due to the similar phenomenological cause behind scattering and the scintillation bandwidth size (Levin et al., 2016). One method of obtaining high resolution dynamic spectra is through cyclic spectroscopy, which recovers the un-scattered pulse by deconvolving the broadened pulse with the exponential tail caused by scattering (Demorest, 2011). Palliyaguru et al. (2015) show that the timing improvement via cyclic spectroscopy is not significant for most MSPs currently observed by NANOGrav, however high resolution dynamic spectra will allow for the accurate recovery of scattering parameters.

Because of the covariances between fitting for DM and scattering, some scattering effects will be absorbed by fitting for only DM. Low-frequency observations can be used in order to disentangle the scattering contributions from the DM as it is easier to discriminate between the $\nu^{-2}$ and $\nu^{-4}$ effects at frequencies where they are more visible. As a result of the change in scattering angle for different observing frequencies, different portions of the ISM are sampled. Cordes et al. (2016) show that DM itself is frequency dependent (i.e. chromatic DM) because of this multipath scattering; using different frequencies to calculate DM results in different DM estimates. This leads to a net difference in dispersive time delays when observing at different frequencies, which in turn increases the uncertainties in DM estimation over wide bandwidths.

Simultaneous dual-frequency observations can improve the PTAs' ability to estimate DM. As discussed above, determining DM from dual frequency observations results in a non-negligible error. Errors can be further decreased by increasing the 


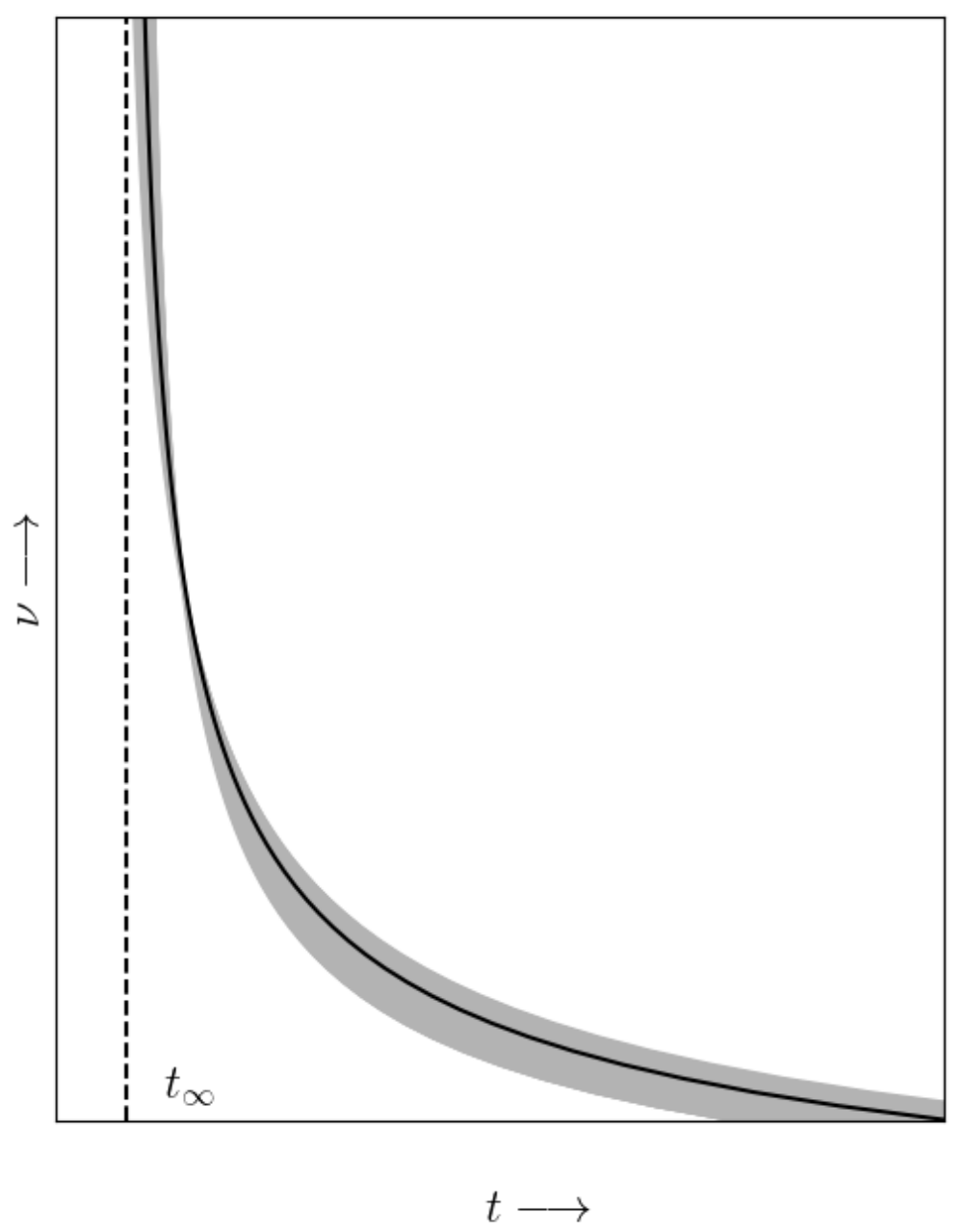

Figure 4.1 DM affecting the pulse arrival time for different frequencies. TOAs at lower frequencies are more affected by dispersion and arrive later than TOAs at higher frequencies. Uncertainties in timing from DM from misestimation correspond to the gray region around the black DM curve. The crossover point on the curve corresponds to some fiducial frequency. 
ratio of observing frequencies. Observing at widely spaced frequencies can aid in obtaining a high precision DM measurement by filling/anchoring part of the DM curve. This more precisely measured DM can then be used to calculate the corrected infinite frequency TOA $t_{\infty}$. A sample DM curve can be seen in Figure 4.1, along with how uncertainties in DM estimation affect the infinite-frequency pulse arrival time.

The increase in precision for DM measured with widely spaced frequencies can be estimated for the data discussed here. Assuming there are no other chromatic timing perturbations, the uncertainty on the estimated DM is

$$
\delta \mathrm{DM} \equiv \mathrm{DM}_{\text {true }}-\mathrm{DM}_{\mathrm{est}}=-\frac{\epsilon_{1}-\epsilon_{2}}{\mathrm{~K}\left(\nu_{1}^{-2}-\nu_{2}^{-2}\right)}
$$

where $K$ is the DM constant, $\epsilon$ is the measurement error on the TOA, $\nu_{1}$ is the lowest observing frequency and $\nu_{2}$ is the highest observing frequency (Cordes et al., 2016). Because of the low frequency coverage of the GMRT, the bottom of Equation 4.3 will be dominated by the GMRT term. For example, if our highest frequency is $\nu_{2}=2300 \mathrm{MHz}$ and we were to go from $\nu_{1}=820 \mathrm{MHz}$ to $\nu_{1}=322 \mathrm{MHz}$, the difference between the TOA measurement errors can be $\sim 4$ times higher and still achieve the same difference between the DMs. If going from $1400 \mathrm{MHz}$ to $322 \mathrm{MHz}$, then the ratio gets even higher — measurement error difference can be 18.9 times higher at lower frequency and still result in the same DM uncertainty. If the errors on the lower frequency data are lower than this level, the $\delta$ DM should decrease. Therefore, lower frequency data are still valuable for estimating DM even if the 
TOA errors are slightly higher.

Along with wider frequency coverage, having an increased number of TOAs is also beneficial for PTAs; a higher cadence of timing observations can improve the possibility of GW detection. The average $\mathrm{S} / \mathrm{N}$ in the intermediate $\mathrm{GW}$ signal limit

$$
\mathrm{S} / \mathrm{N} \propto N_{\mathrm{MSP}} T^{1 / 2}\left(\frac{c}{\sigma_{\mathrm{RMS}}^{2}}\right)^{3 / 26}
$$

where $N_{\mathrm{MSP}}$ is the number of MSPs in the data set, $T$ is the length of the data span, $\sigma_{\mathrm{RMS}}$ is the timing RMS and $c$ is the cadence of observations. Having more frequent observations will improve the $\mathrm{S} / \mathrm{N}$ of PTA data. The TOA RMS goes down by a factor of $\sqrt{N_{o b s}}$ over time for pulsars where the red noise limit has not been reached; more observations could lower the white noise for pulsars. When the white noise becomes low, the red noise becomes the limiting factor.

Frequency independent red noise, like pulsar spin noise or external influences like an asteroid belt (Shannon et al., 2013), can be modeled but cannot be corrected for in the timing. A gravitational wave signal with a period of several years appears as a low-frequency $(\mathrm{nHz})$ red noise process that is independent of radio frequency. Radio frequency dependent red noise, such as from interstellar propagation effects, will appear as a systematic frequency dependent offset in TOAs if not properly corrected for. When frequency dependent red noise processes are not properly mitigated, they present partially as frequency independent processes and can be covariant with a GW signal, making GW detection more difficult. If the goal is to detect GWs using pulsar timing, then PTAs need to optimize their ability to 
Table 4.1 NANOGrav Observing Frequencies

\begin{tabular}{llcccc}
\hline \hline PSR & \multicolumn{5}{c}{ Observing frequencies } \\
& \multicolumn{5}{c}{ MHz } \\
\hline J0030+0451 & $\ldots$ & 430 & $\ldots$ & 1400 & $\ldots$ \\
$\mathrm{J} 1640+2224$ & $\ldots$ & 430 & $\ldots$ & 1400 & $\ldots$ \\
$\mathrm{J} 1713+0747$ & $\ldots$ & $\ldots$ & 820 & 1400 & 2300 \\
$\mathrm{~J} 1738+0333$ & $\ldots$ & $\ldots$ & $\ldots$ & 1400 & 2300 \\
$\mathrm{~B} 1855+09$ & $\ldots$ & 430 & $\ldots$ & 1400 & $\ldots$ \\
$\mathrm{J} 1909-3744$ & $\ldots$ & $\ldots$ & 820 & 1400 & $\ldots$ \\
$\mathrm{B} 1937+21$ & $\ldots$ & $\ldots$ & 820 & 1400 & 2300 \\
$\mathrm{~J} 2017+0603$ & $\ldots$ & 430 & $\ldots$ & 1400 & 2300 \\
$\mathrm{~J} 2145-0750$ & $\ldots$ & $\ldots$ & 820 & 1400 & $\ldots$ \\
$\mathrm{J} 2214+3000$ & $\ldots$ & $\ldots$ & $\ldots$ & 1400 & 2300 \\
$\mathrm{~J} 2317+1439$ & 327 & 430 & $\ldots$ & 1400 & $\ldots$ \\
\hline \hline
\end{tabular}

Notes. Observing frequencies for MSPs that are discussed in this paper, observed with the Arecibo and Green Bank observatories from the NANOGrav 11-year data set (Arzoumanian et al., 2018b).

characterize and model other red noise processes like DM in the data.

The Giant Metre-wave Radio Telescope (GMRT) is located $80 \mathrm{~km}$ north of Pune, India. The array consists of 30 45-meter stationary antennas. Using six feeds, the array can observe frequencies ranging from 150 to $1500 \mathrm{MHz}$. The GMRT has a dual feed receiver capable of executing simultaneous dual-frequency observations that can be complementary to NANOGrav observations by offering coverage at lower frequencies.

We present timing data for 10 MSPs obtained using the GMRT. There are several questions we want to answer with these data. Are the DMs measured at high and low frequencies consistent, and what constraint can we place on DMs that differ? Can this coverage at low frequency improve DM measurements, with better precision than those attained with the Arecibo Observatory? We discuss the data 
and acquisition system at the GMRT in Section 4.3. We examine data taken with the GMRT along with NANOGrav observations to compare DM measurements in Section 4.4. We discuss its potential for producing higher precision DMs in Section 4.5 .

\subsection{Data}

Data were taken simultaneously centered at $322 \mathrm{MHz}$ and $607 \mathrm{MHz}$ with a $32 \mathrm{MHz}$ bandwidth at each frequency using the phased array mode. Observations occurred at 11 epochs between February 2nd 2013 and May 12th 2014. A test pulsar B1929+10 was observed at each epoch for $\sim 5$ minutes. The GMRT Software Backend simultaneously creates both coherently and incoherently dedispersed data (Roy et al., 2010). The analysis was performed on the coherently dedispersed pulsar data. Data were split into 32 sub-bands across each band. No flux or polarization calibrations were done during these observations. The data headers also have to be inserted after the observation using a separate script as they are not encoded during the observation. Clock correction files do not exist for the GMRT, and were thus not used in the timing. Observation information is recorded in Table 4.2.

Data were folded using parfiles obtained from $\operatorname{PSRCAT}^{1}$. Multiple gaussian fits were used for each pulsar at each frequency for the epoch producing the highest signal-to-noise $(\mathrm{S} / \mathrm{N})$ observation in order to produce a pulse template for calculating TOAs. PSRCHIVE was used for TOA generation (van Straten et al., 2012). Fitting

\footnotetext{
${ }^{1}$ http://www.atnf.csiro.au/people/pulsar/psrcat/
} 


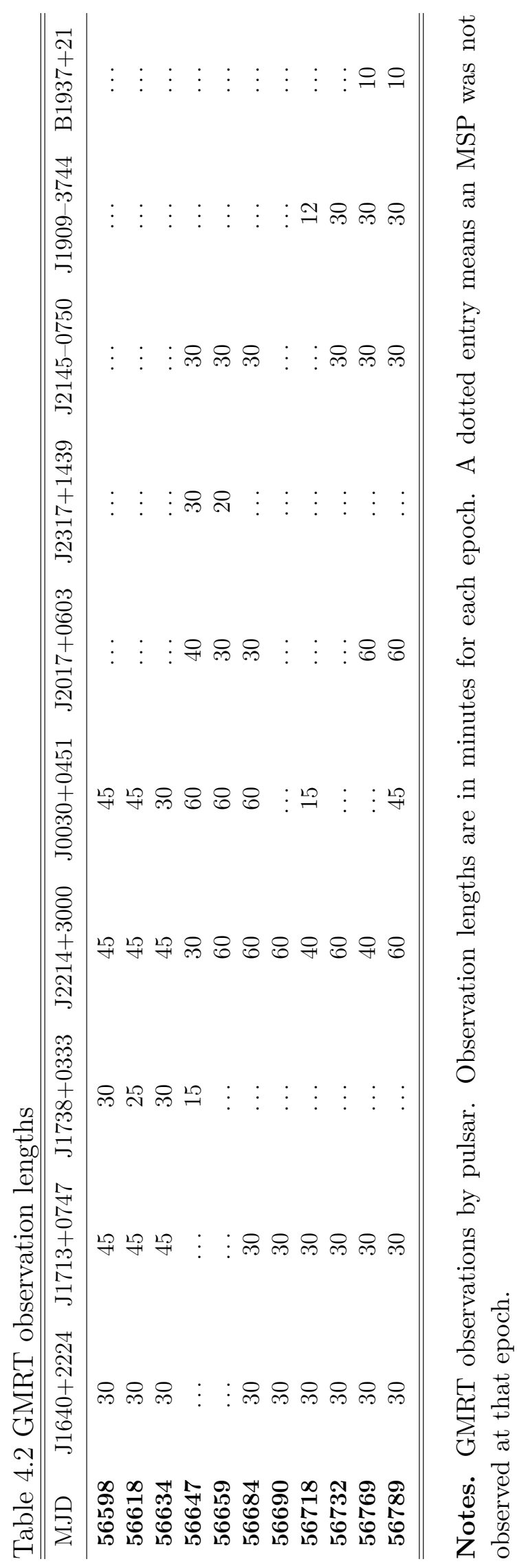


was done using the TEMPO software package ${ }^{2}$, which applies a least-squares fit to the data. Errors on DM are $1 \sigma$ and are determined from the timing-parameter covariance matrix after the least-squares timing model fit. Timing was performed using parameter files produced from the NANOGrav 11-year data set (Arzoumanian et al., 2018b). The NANOGrav parameter values are more sensitive than those we would obtain from fitting just the GMRT data, therefore all parameters except DM were held constant in order to obtain a DM estimate while analyzing the GMRT data.

Observation frequencies for the NANOGrav data can be seen in Table 4.1 for comparison. GMRT data could provide low frequency coverage in complement with the NANOGrav data, and fill in the gaps where data below $820 \mathrm{MHz}$ does not exist in the 11-year data set.

\subsection{Comparison of DM measurements}

GMRT DM measurements obtained from timing can be seen in Table 4.3 alongside DMs measured from the NANOGrav 11-year data. DMs were obtained at $322 \mathrm{MHz}, 607 \mathrm{MHz}$, and then a joint fit for both frequencies for comparison. DMs from the NANOGrav data and the dual-frequency GMRT data sets are also plotted in Figure 4.2. Even with the larger errors on the GMRT DM estimates, the values are not consistent for many epochs.

There are several reasons why DM measurements would differ between the data sets. Non-simultaneous measurements between the different observatories could

\footnotetext{
${ }^{2}$ TEMPO software package: http://tempo.sourceforge.net
} 


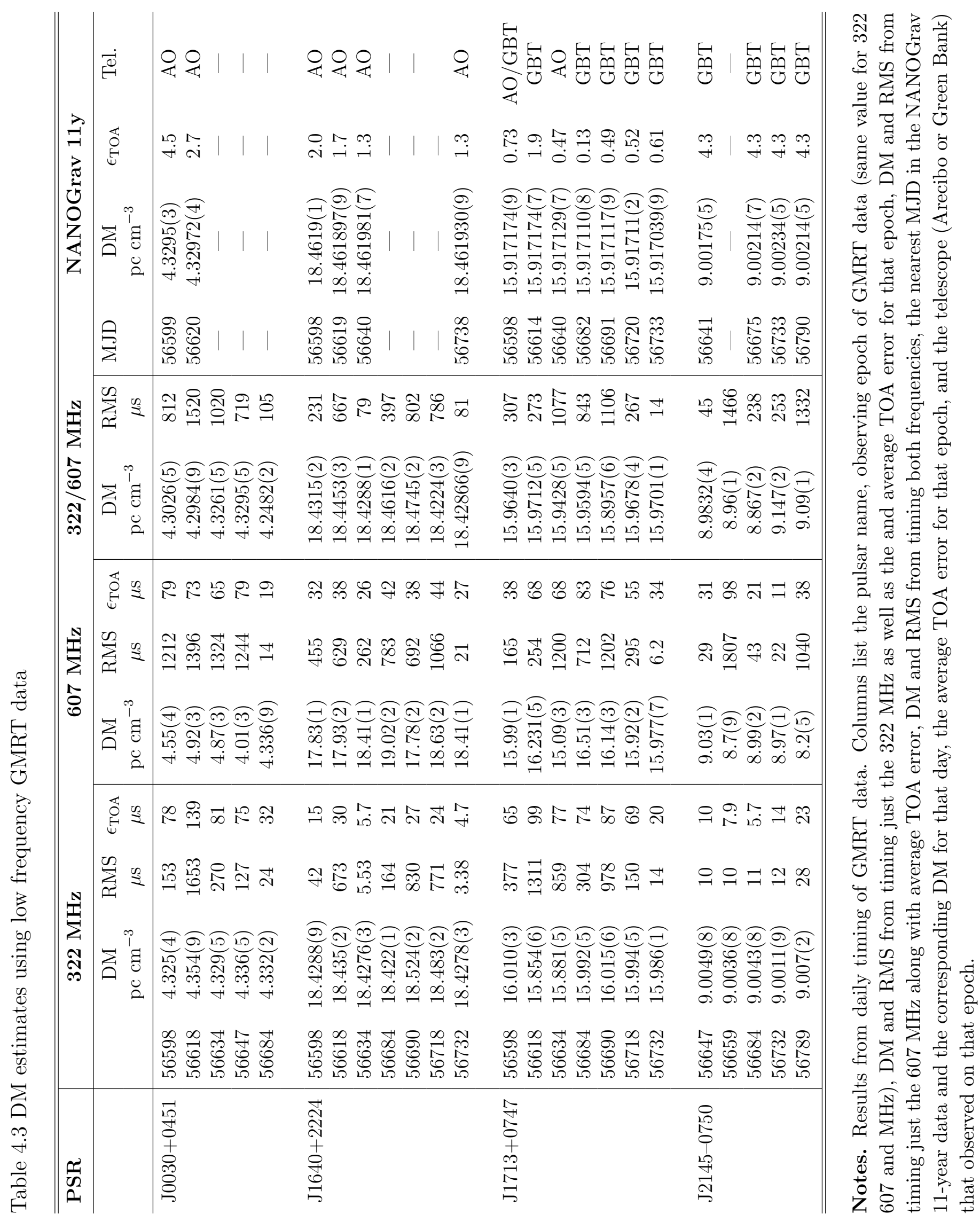



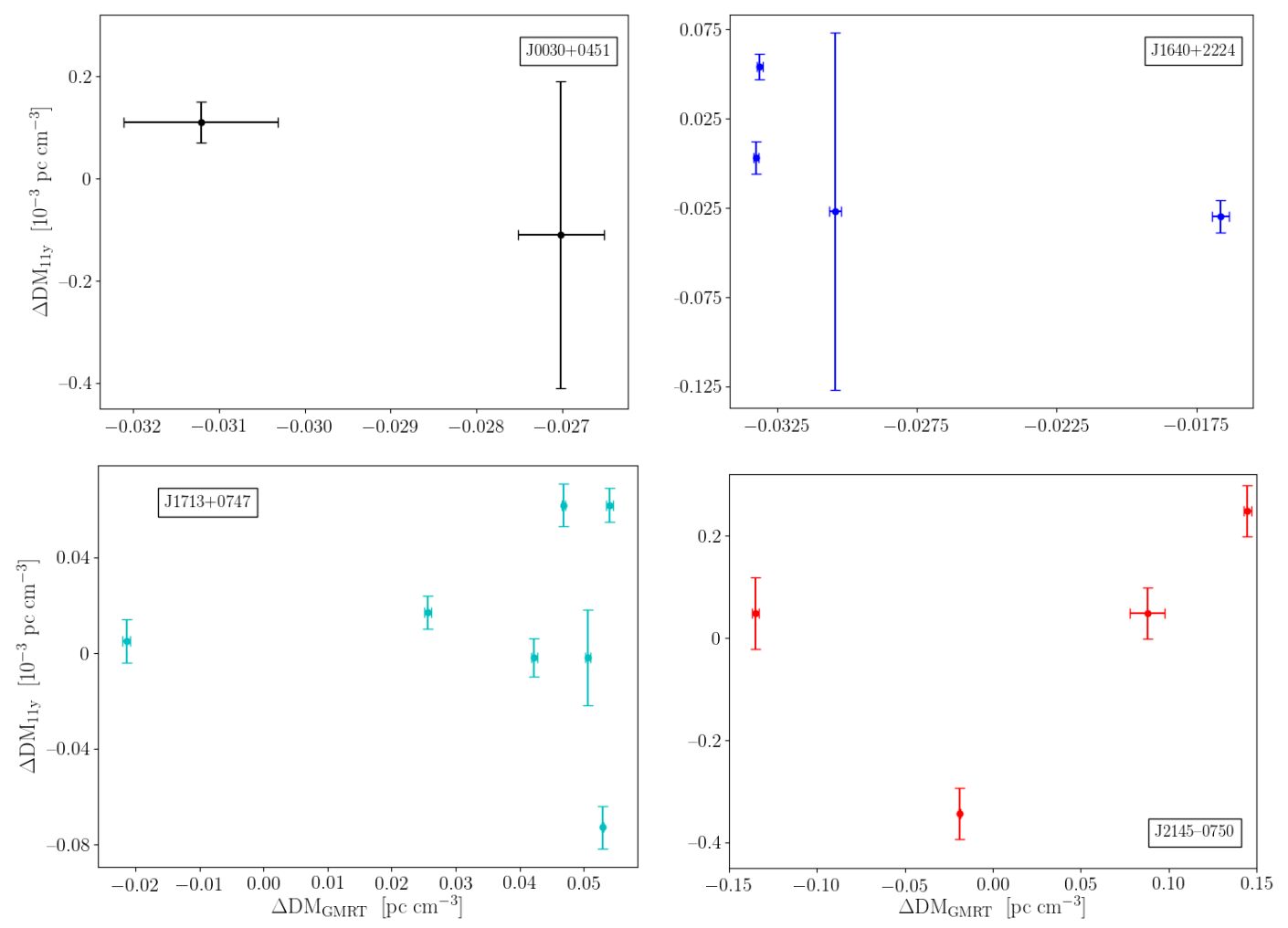

Figure 4.2 DM comparison from timing. DM measurements obtained from the combined 322/607 MHz GMRT data are shown on the x-axis, while DMs from the NANOGrav 11-year data are on the y-axis. Note that the two axes are not to the same scale due to the high precision of the NANOGrav measurements. If the estimates matched, they would sit at 0 , signifying a 1:1 relation. 
cause discrepancies. Some of DM measurements have $\sim$ week differences between epochs. Jones et al. (2017) calculate the timescales it takes for the DM to vary beyond the measurement errors. The DM variation timescales for the pulsars timed here are all greater than one month, so this is unlikely to be the reason for the discrepancies. Lam et al. (2016) modeled ionospheric effects and place an upper limit of their DM contribution of $\sim 10^{-4} \mathrm{pc} \mathrm{cm}^{-3}$, two orders of magnitude smaller than all of the DM differences seen in Table 4.3. The largest time between epochs is 9 days, making the upper limit on such a change in DM $\sim 6 \times 10^{-6} \mathrm{pc} \mathrm{cm}^{-3}$ (the scaling over time is sub-linear). Following Equation 12 in Cordes et al. (2016) which assumes scattering is due to a thin screen, a fiducial pulsar observed at 322 and 607 $\mathrm{MHz}$ would result in an RMS DM offset due to chromatic DMs of $\sim 10^{-4} \mathrm{pc} \mathrm{cm}^{-3}$. Correcting for only DM and not scattering will cause discrepancies as the DM fit will absorb some scattering effects. However, all four MSPs have DMs below 19 pc $\mathrm{cm}^{-3}$, so they likely do not show significant amounts of scattering. Lam et al. (2015) estimate the expected DM change per day from a strictly Kolmogorov medium of

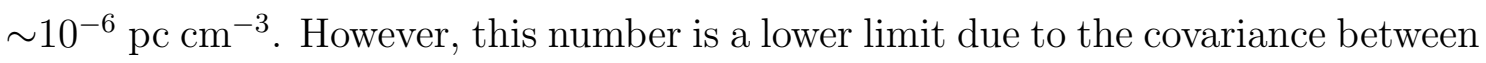
the power spectrum of a Kolmogorov medium and the presence of a DM trend; the presence of a trend would results in a slightly higher DM change.

Here we discuss the DMs for each MSP in more detail, which can be seen in Table 4.3. DM trends listed below were calculated for NANOGrav 9-year data set and discussed by Jones et al. (2017). 


\subsubsection{PSR J0030+0451}

For MJD 56598, PSR J0030+0451 has a mean TOA uncertainty of $\epsilon_{\mathrm{TOA}}=$ $78 \mu s$ at $322 \mathrm{MHz}$. Following Equation 4.3, the expected uncertainty in DM when just using the $322 \mathrm{MHz}$ data is $\delta \mathrm{DM}=0.003 \mathrm{pc} \mathrm{cm}^{-3}$. For comparison, the actual DM uncertainty for this day is $0.004 \mathrm{pc} \mathrm{cm}^{-3}$, which agrees with the predicted $\delta \mathrm{DM}$ value. By combining the $322 \mathrm{MHz}$ and NANOGrav data (where $\nu=1400 \mathrm{MHz}$ for this epoch), the expected DM uncertainty becomes $\delta \mathrm{DM}=4 \times 10^{-4} \mathrm{pc} \mathrm{cm}^{-3}$ whereas the NANOGrav data alone has a DM uncertainty of $3 \times 10^{-4} \mathrm{pc} \mathrm{cm}^{-3}$. For this particular MJD, adding in the $322 \mathrm{MHz}$ data neither helps nor hurts DM uncertainty as the two values are comparable. Other MJDs have a lower average TOA uncertainty, but did not have a NANOGrav observation within a few weeks that could be used for a comparison.

A linear trend was not found for PSR J0030+0451.A periodic component was found with a peak amplitude of $1.2 \pm 0.3 \times 10^{-4} \mathrm{pc} \mathrm{cm}^{-3}$. This DM variation amplitude is an order of magnitude smaller than the variations between DMs seen for this pulsar at $322 \mathrm{MHz}$ and two orders of magnitude smaller than those at 607 $\mathrm{MHz}$. It is therefore not feasible that this periodicity is causing the variation seen between DMs.

\subsubsection{PSR J1640+2224}

Using timing data from MJD 56732, PSR J1640+2224 has a mean TOA uncertainty of $\epsilon_{\mathrm{TOA}}=4.7 \mu \mathrm{s}$ at $322 \mathrm{MHz}$. The expected uncertainty in DM when 
just using the $322 \mathrm{MHz}$ is $\delta \mathrm{DM}=2 \times 10^{-4} \mathrm{pc} \mathrm{cm}^{-3}$. The actual DM uncertainty for this day is $3 \times 10^{-4} \mathrm{pc} \mathrm{cm}^{-3}$, which agrees with the predicted value. By combining the $322 \mathrm{MHz}$ and NANOGrav data (where $\nu=1400 \mathrm{MHz}$ ), the expected DM uncertainty becomes $\delta \mathrm{DM}=2 \times 10^{-5} \mathrm{pc} \mathrm{cm}^{-3}$; for comparison, the NANOGrav data alone is $9 \times 10^{-6} \mathrm{pc} \mathrm{cm}^{-3}$, which means the two values are fairly similar. The majority of TOA uncertainties for other epochs at both 322 and $607 \mathrm{MHz}$ are $\sim$ an order of magnitude larger the uncertainty for this epoch, so it is unlikely that adding in other days would improve the DM uncertainty.

A linear trend was calculated to be $d \mathrm{DM} / d t=1.45 \pm 0.03 \times 10^{-2} \mathrm{pc} \mathrm{cm}^{-3}$ $\mathrm{yr}^{-1}\left(4.0 \pm 0.08 \times 10^{-5} \mathrm{pc} \mathrm{cm}^{-3} \mathrm{day}^{-1}\right)$. This value is too small to account for the DM changes seen over $\sim 20$ days between observing epochs, so a linear trend cannot account for these variations.

\subsubsection{PSR J1713+0747}

For MJD 56732, PSR J1713+0747 shows a mean TOA uncertainty of $\epsilon_{\mathrm{TOA}}=$ $20 \mu s$ at $322 \mathrm{MHz}$. The expected uncertainty in DM when just timing the $322 \mathrm{MHz}$ data are $\delta \mathrm{DM}=9 \times 10^{-4} \mathrm{pc} \mathrm{cm}^{-3}$. The actual DM uncertainty for this epoch is $0.001 \mathrm{pc} \mathrm{cm}^{-3}$, which agrees with the predicted value. By combining the $322 \mathrm{MHz}$ and NANOGrav data (where $\nu=1400 \mathrm{MHz}$ ), the expected DM uncertainty becomes $\delta \mathrm{DM}=9 \times 10^{-5} \mathrm{pc} \mathrm{cm}^{-3}$. Timing the NANOGrav data alone gives a DM uncertainty of $9 \times 10^{-6} \mathrm{pc} \mathrm{cm}^{-3}$, an order of magnitude more precise than that calculated from including the low frequency data. The TOA uncertainty is $2-3$ orders of magnitude 
larger for other epochs at both 322 and $607 \mathrm{MHz}$, therefore incorporating more of the current low frequency data will not improve the precision of DM measurements for this MSP.

PSR J1713+0747 was found to show a linear trend of $d \mathrm{DM} / d t=-6.6 \pm 0.8 \times 10^{-5}$ pc $\mathrm{cm}^{-3} \mathrm{yr}^{-1}\left(-1.8 \pm 0.2 \times 10^{-7} \mathrm{pc} \mathrm{cm}^{-3}\right.$ day $\left.^{-1}\right)$ as well as a periodic component with a peak amplitude of $5 \pm 1 \times 10^{-5} \mathrm{pc} \mathrm{cm}^{-3}$. These trends are much too small to account for the variations in DMs seen here. The DMs are not varying monotonically, which is uncharacteristic for a linear trend but is expected for a periodicity; however the DM variations are still $\sim 2$ orders of magnitude too large to be accounted for by this periodic component.

\subsubsection{PSR J2145-0750}

For MJD 56684, PSR J2145-0750 has a mean TOA uncertainty of $\epsilon_{\mathrm{TOA}}=$ $5.7 \mu s$ at $322 \mathrm{MHz}$. The expected uncertainty in DM when just using the $322 \mathrm{MHz}$ data are $\delta \mathrm{DM}=2.5 \times 10^{-4} \mathrm{pc} \mathrm{cm}^{-3}$. The actual $\mathrm{DM}$ uncertainty for this epoch is $8 \times 10^{-4} \mathrm{pc} \mathrm{cm}^{-3}$, which is comparable to the predicted value. By combining the $322 \mathrm{MHz}$ and NANOGrav data (where $\nu=820 \mathrm{MHz}$ for this epoch), the expected DM uncertainty becomes $\delta \mathrm{DM}=3 \times 10^{-5} \mathrm{pc} \mathrm{cm}^{-3}$. The NANOGrav data alone has a DM uncertainty of $7 \times 10^{-5} \mathrm{pc} \mathrm{cm}^{-3}$; this is the only MSP for which adding in the $322 \mathrm{MHz}$ data in its current state will improve the uncertainty on DM (data at 607 $\mathrm{MHz}$ is much less precise on two epochs, and comparable for three epochs). PSR J2145-0750 exhibits a linear trend of $d \mathrm{DM} / d t=8 \pm 2 \times 10^{-5} \mathrm{pc} \mathrm{cm}^{-3}$ 


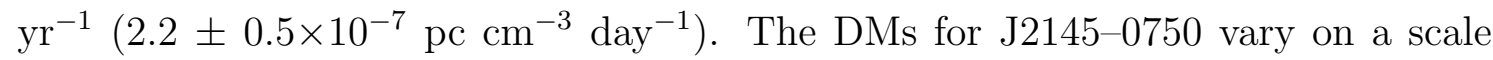
$\sim 2$ orders of magnitude higher than this. In addition, the DM values do not vary monotonically, so a linear trend is not the cause of these varying DMs.

\subsection{Conclusions}

The 9-year data set shows DM variations on the scale of the sources of varying DM discussed in $§ 4.4$. The DMs measured for the GMRT show variations much greater than these, which suggests that the reasons for these variations are not astrophysical.

For all of the MSPs discussed above, previously measured DM trends could not account for the variations seen between DM measurements. A DM variation amplitude much larger than would be expected given the average Galactic free electron density could indicate that the MSP is located in an overdense environment or is ionizing the material in the ISM along its path. However, it is unlikely that all of the pulsars discussed here are sitting in localized overdense regions, and this explanation does not account for the large difference in DMs measured between the GMRT and NANOGrav data. Due to chromatic DMs, we would not expect agreement between DMs measured at different frequencies, but as discussed above these difference are outside the range possible if caused by chromatic DMs.

For one MSP, PSR J2145-0750, incorporating the low frequency data did lower the predicted DM uncertainty when combined with NANOGrav data. However, this prediction is based solely on the mean TOA uncertainties and as explained above 
Table 4.4 Sky and system temperatures

\begin{tabular}{lcrc}
\hline \hline PSR & $\begin{array}{c}\mathrm{T}_{\text {sky }} \\
\mathrm{K}\end{array}$ & $\begin{array}{c}\text { Flux } \\
\mathrm{mJy}\end{array}$ & $\begin{array}{c}\mathrm{T}_{\text {sys }} / \mathrm{G} \\
\mathrm{K} /(\mathrm{K} / \mathrm{Jy})\end{array}$ \\
\hline $\mathrm{J} 0030+0451$ & 41 & 1.4 & 211 \\
$\mathrm{~J} 1640+2224$ & 60 & 1.0 & 43 \\
$\mathrm{~J} 1713+0747$ & 128 & 6.3 & 516 \\
$\mathrm{~J} 2145-0750$ & 46 & 3.2 & 30 \\
\hline
\end{tabular}

Notes. Sky and system temperatures for GMRT pulsars. Spectral index values were assumed to be $\alpha=-1.6$ (Jankowski et al., 2018). Flux density measurements are for 1400 MHz. System temperatures were calculated via Equation 4.5.

does not explain why the DMs between frequencies are so variant. None of the MSPs that we calculated DMs for were observed at $2300 \mathrm{MHz}$ by NANOGrav; conducting this analysis with MSPs for which NANOGrav has measured TOAs at this higher frequency could produce a higher precision DM estimate when combined with low frequency data.

The ratio of the system temperature $T_{\text {sys }}$ and the gain $G$ can be calculated by

$$
\frac{T_{\text {sys }}}{G}=\sigma_{\mathrm{RMS}} \sqrt{2 \Delta \nu \Delta t}
$$

where $\sigma_{\mathrm{RMS}}$ is the off pulse RMS, $\Delta \nu$ is the observing bandwidth and $\Delta t$ is the number of seconds per channel $\left(t_{\text {obs }} / n_{\text {bins }}\right)$. The expected flux at 322 and $607 \mathrm{MHz}$ can be scaled from other know values at other frequencies; scaling factors for a few of these pulsars can be seen in Table 4.4 along with calculated system temperatures.

We are not currently obtaining better DM measurements with the GMRT data. As outlined in previous sections, there are some missing pieces to the data that could improve the timing and make it useful for inclusion into the IPTA. As 
mentioned in Section 4.3, polarization calibration was not done when the GMRT data used here were obtained. Not accounting for polarization causes TOA uncertainties due to deviations from the pulse profile template. Using fiducial values for NANOGrav data, Lam et al. (2018) calculate the TOA uncertainty induced by errors in polarization calibration to be $\sim 1 \mu$ s for narrow frequency channels (this value averages down per observation, but may change systematically between epochs); as no polarization calibration was performed, the errors for the GMRT data are likely larger. The reason for the large errors currently seen in the GMRT data are unclear. Uncertainties like these would need to be mitigated before inclusion into the IPTA. 


\section{Chapter 5}

\section{Conclusion}

We have utilized multiple radio telescopes to investigate astrophysics related to the detection of GWs using PTAs. We used data from the Arecibo and Green Bank observatories to study scale and structure in DM variations, and to characterize components in the ISM. We analyzed VLA data to follow up on a (relatively) nearby SMBH binary candidate that could be a detectable GW source in the future. We compared DM measurements obtained with the GMRT to those measured by NANOGrav to assess their precision and the possibility of including the GMRT into IPTA efforts. Here we summarize the importance of these works, future works that can be done, and future directions for PTAs as a whole.

\subsection{Importance of DM characterization and understanding the ISM}

We examined a large set of DM timeseries from the NANOGrav 9-year data set and found evidence of timescales as small as weeks of variations in the vast majority of MSPs. We found evidence of linear trends and annual periodicities, in addition to evidence for discrete ISM structures. We calculated and interpreted structure functions (SFs) for a subset of MSPs and examined DM variations due to LOS motions. These SFs often appear non-Kolmogorov, but we showed that this can be due to the presence of trends in the data and does not necessarily indicate a 
non-Kolmogorov medium.

As stated in previous sections, DM characterization is crucial for PTA success in the search for GWs. Monitoring DM gives us information about the ISM along the LOS, specifically the free electron density. The changing LOS over time that causes trends in DM can inform us on structure in the ISM. Understanding structure in the ISM and being able to distinguish ISM effects from other noise will help us form a more complete model of the time delays present in PTA data. More telescopes with a wider frequency range, like the GMRT, and more frequent observations (like CHIME's ability to get daily DMs) will improve our ability to measure and correct for DM and monitor variations.

\subsection{Investigating a potential SMBH binary candidate}

We investigated the hypothesis that the central SMBH in NGC 3115 was in fact offset from the galactic center, which could represent a single offset active galactic nucleus (AGN) or an AGN in orbit around a central black hole. We analyzed $10 \mathrm{GHz}$ VLA data and found evidence for only one AGN. We placed a limit on the radio luminosity of any secondary supermassive black hole. We analyzed the relative positioning of the radio core, X-ray nucleus, and stellar bulge in this galaxy and determined that the radio source is centrally located with no offset from the galactic bulge.

NGC 3115 is a relatively nearby galaxy; if a SMBH binary was confirmed, it would be a potential future GW source for PTAs. Once GWs are detected with 
PTAs, multi-messenger observations will give additional information about astronomical events that we have never had before and can aid in identifying possible sources that in the future may be GW emitters. One difficulty in investigating these sources is the comparison of reference frames between wavelengths and data sets. Each observation and instrument has its own astrometric offset (e.g. from the International Celestial Reference Frame); accurately accounting for these differences in reference frames can be complex.

\subsubsection{Data combination and PTA data standards}

We discussed the importance of lower frequency coverage as well as simultaneous dual-frequency observations available at the GMRT, and its potential to provide higher precision dispersion measurements than the data taken by NANOGrav. We compared the DMs obtained through both data sets. We discussed the possible advantages of incorporating GMRT data into timing efforts, as well as anticipated challenges.

There are several telescopes starting pulsar timing now, and many more that are anticipated to be useful instruments for pulsar timing. With more telescopes comes higher cadence, more TOAs, better frequency coverage, and improved sky coverage. Some future telescopes will be larger and/or have more advanced electronics, and will surpass current observatories in sensitivity. Incorporating these additional instruments will increase GW sensitivity.

As more telescopes come online in the next few years, along with new PTAs 
forming that may join the IPTA, establishing minimum data standards and uniformity in data acquisition/recording will become necessary, particularly when those working on data combination efforts are not familiar with a specific telescope. PTAs must establish a minimal documentation requirement for observers or data processors to use to avoid reinventing the wheel each time data change hands. It would also establish a norm so that all data used for the same purpose would be processed the same way to avoid discrepancies in different pipelines.

There are several implementations that could be made to improve data processing. There is a difference between the terrestrial time standard (that must be converted to the Solar System barycenter) and the observatory clocks; this offset can be corrected for in the timing using a clock correction. These time corrections are required to avoid a systematic offset for every TOA measured at that observatory.

Headers must be recorded for every observation to avoid confusion and an opportunity for mistakes during the processing. The headers must include information like the pulsar name, RA and Dec, observing frequency, and the offset from UTC that needs to be added to the timestamp. For the GMRT, timestamp information is recorded in Indian Standard Time (IST) that must be converted to UTC. Errors in the header may be overridden in PSRCHIVE if necessary. A standard requirement of creating and incorporating headers in data would alleviate concerns about incorrect header information and the necessity to manually update each data header.

Polarization calibration must be done for all observations; errors in calibration (or an absence of calibration) introduce uncertainty to the timing residuals. As mentioned above, different observatories use different hardware and software; cali- 
bration observations when observing pulsars are very important for correcting and assessing data from multiple sites.

\subsection{Going forward}

We are entering a new era in GW astronomy. More telescopes are coming online with wideband systems which will not only improve our ability to measure DM but also increase TOA sensitivity. As time goes on, pulsar searches will find more MSPs which may be viable for PTA inclusion. For certain types of GW sources, a few well-timed pulsars are more useful than having many pulsars (e.g. continuous waves or bursts with memory). PTAs will become more sensitive as time goes on; certain telescopes will bring specific advantages, which can be exploited to maximize our sensitivity for searches for a particular GW source. Data combination will result in more data accessible to the IPTA as well as more algorithms for data processing and analysis.

PTAs are likely to detect GWs from the stochastic background in the next $\sim 5$ years. NANOGrav is already probing into the region of strain amplitude phase space where we expect GWs to exist. Placing these constraints can inform on the SMBH binary environments, like gas/stellar content and orbital eccentricity. PTAs also put limits on cosmic string tension that are more precise than other experiments such as Planck. PTAs will continue to increase sensitivity and improve limits, and by doing so will explore a new frontier in the GW universe not accessible by other experiments. The detection of low-frequency GWs will be another step towards 
the characterization of our Universe. We have entered the era of GW astronomy, where GW and electromagnetic emission can be used to learn more about exotic astronomical events than has ever been possible before. 


\section{Bibliography}

Andrae, R., Schulze-Hartung, T., \& Melchior, P. 2010, ArXiv e-prints, arXiv:1012.3754

Antoniadis, J. I. 2013, PhD thesis, University of Bonn

Arzoumanian, Z., Brazier, A., Burke-Spolaor, S., et al. 2015, ApJ, 813, 65

Arzoumanian, Z., Baker, P. T., Brazier, A., et al. 2018a, ApJ, 859, 47

-. 2018b, ApJ, 859, 47

Backer, D. C., Kulkarni, S. R., Heiles, C., Davis, M. M., \& Goss, W. M. 1982, Nature, 300, 615

Begelman, M. C., Blandford, R. D., \& Rees, M. J. 1980, Nature, 287, 307

Bhat, N. D. R., Cordes, J. M., Camilo, F., Nice, D. J., \& Lorimer, D. R. 2004, ApJ, 605,759

Blecha, L., Civano, F., Elvis, M., \& Loeb, A. 2013, MNRAS, 428, 1341

Burke-Spolaor, S. 2011, MNRAS, 410, 2113

Campanelli, M., Lousto, C. O., Zlochower, Y., \& Merritt, D. 2007, Physical Review Letters, 98, 231102

Cantiello, M., Capaccioli, M., Napolitano, N., et al. 2015, A\&A, 576, A14

Cantiello, M., D'Abrusco, R., Spavone, M., et al. 2018, A\&A, 611, A93 
Chatterjee, S., \& Cordes, J. M. 2004, ApJL, 600, L51

Chiaberge, M., Ely, J. C., Meyer, E. T., et al. 2017, A\&A, 600, A57

Clegg, A. W., Fey, A. L., \& Lazio, T. J. W. 1998, ApJ, 496, 253

Cordes, J. M. 2002, in Astronomical Society of the Pacific Conference Series, Vol. 278, Single-Dish Radio Astronomy: Techniques and Applications, ed. S. Stanimirovic, D. Altschuler, P. Goldsmith, \& C. Salter, 227-250

Cordes, J. M., \& Lazio, T. J. W. 2002, ArXiv Astrophysics e-prints, arXiv:astro$\mathrm{ph} / 0207156$

Cordes, J. M., \& Rickett, B. J. 1998, ApJ, 507, 846

Cordes, J. M., \& Shannon, R. M. 2010, ArXiv e-prints, arXiv:1010.3785

Cordes, J. M., Shannon, R. M., \& Stinebring, D. R. 2016, ApJ, 817, 16

Cordes, J. M., Wolszczan, A., Dewey, R. J., Blaskiewicz, M., \& Stinebring, D. R. 1990, ApJ, 349, 245

Cutri, R. M., Skrutskie, M. F., Van Dyk, S., et al. 2003, Explanatory Supplement to the 2MASS All Sky Data Release and Extended Mission Products, , nASA/IPAC Infrared Science Archive, http://www.ipac.caltech.edu/2mass/releases/allsky/doc/

Demorest, P. B. 2011, MNRAS, 416, 2821

Demorest, P. B., Ferdman, R. D., Gonzalez, M. E., et al. 2013, ApJ, 762, 94 
Desvignes, G., Caballero, R. N., Lentati, L., et al. 2016, MNRAS, 458, 3341

Dolch, T., Lam, M. T., Cordes, J., et al. 2014, ApJ, 794, 21

Einstein, A. 1915, Sitzungsber. preuss.Akad. Wiss., vol. 47, No.2, pp. 831-839, 1915, 47,831

Evans, I. N., Primini, F. A., Glotfelty, K. J., et al. 2010, ApJS, 189, 37

Fonseca, E., Stairs, I. H., \& Thorsett, S. E. 2014, ApJ, 787, 82

Frisch, P. C., Redfield, S., \& Slavin, J. D. 2011, ARA\&A, 49, 237

Gültekin, K., Richstone, D. O., Gebhardt, K., et al. 2009, ApJ, 698, 198

Hamilton, P. A., Hall, P. J., \& Costa, M. E. 1985, MNRAS, 214, 5P

He, C., Ng, C.-Y., \& Kaspi, V. M. 2013, ApJ, 768, 64

Hellings, R. W., \& Downs, G. S. 1983, ApJL, 265, L39

Hewish, A., Bell, S. J., Pilkington, J. D. H., Scott, P. F., \& Collins, R. A. 1968, Nature, 217, 709

Hobbs, G., Dai, S., Manchester, R. N., et al. 2014, ArXiv e-prints, arXiv:1407.0435

Hulse, R. A., \& Taylor, J. H. 1975, ApJL, 195, L51

Isaacman, R., \& Rankin, J. M. 1977, ApJ, 214, 214

Jankowski, F., van Straten, W., Keane, E. F., et al. 2018, MNRAS, 473, 4436

Jenet, F. A., Hobbs, G. B., Lee, K. J., \& Manchester, R. N. 2005, ApJL, 625, L123 
Johnston, S., Nicastro, L., \& Koribalski, B. 1998, MNRAS, 297, 108

Jones, M. L., McLaughlin, M. A., Lam, M. T., et al. 2017, ApJ, 841, 125

Kaplan, D. L., Kupfer, T., Nice, D. J., et al. 2016, ApJ, 826, 86

Kaspi, V. M., Taylor, J. H., \& Ryba, M. F. 1994, ApJ, 428, 713

Keith, M. J., Coles, W., Shannon, R. M., et al. 2013, MNRAS, 429, 2161

Komossa, S. 2012, Advances in Astronomy, 2012, 364973

Komossa, S., Zhou, H., \& Lu, H. 2008, ApJL, 678, L81

Kormendy, J., \& Richstone, D. 1992, ApJ, 393, 559

Lacey, C., \& Cole, S. 1993, MNRAS, 262, 627

Lam, M. T., Cordes, J. M., Chatterjee, S., \& Dolch, T. 2015, ApJ, 801, 130

Lam, M. T., Cordes, J. M., Chatterjee, S., et al. 2016, ApJ, 821, 66

Lam, M. T., McLaughlin, M. A., Cordes, J. M., Chatterjee, S., \& Lazio, T. J. W. 2018, ApJ, 861, 12

Lena, D., Robinson, A., Marconi, A., et al. 2014, ApJ, 795, 146

Lentati, L., Kerr, M., Dai, S., et al. 2017, ArXiv e-prints, arXiv:1703.02108

Lentati, L., Shannon, R. M., Coles, W. A., et al. 2016, MNRAS, 458, 2161

Levin, L., McLaughlin, M. A., Jones, G., et al. 2016, ApJ, 818, 166 
Lin, D., Irwin, J. A., Wong, K.-W., et al. 2015, ApJ, 808, 19

Lorimer, D. R., \& Kramer, M. 2012, Handbook of Pulsar Astronomy

Lützgendorf, N., Helm, E. v. d., Pelupessy, F. I., \& Portegies Zwart, S. 2016, MNRAS, 456, 3645

Manchester, R. N., Hobbs, G. B., Teoh, A., \& Hobbs, M. 2005, AJ, 129, 1993

Manchester, R. N., \& IPTA. 2013, Classical and Quantum Gravity, 30, 224010

Manchester, R. N., Hobbs, G., Bailes, M., et al. 2013, PASA, 30, e017

Margutti, R., Berger, E., Fong, W., et al. 2012, ApJ, 756, 63

Matthews, A. M., Nice, D. J., Fonseca, E., et al. 2016, ApJ, 818, 92

McLaughlin, M. A. 2013, Classical and Quantum Gravity, 30, 224008

Menezes, R. B., Steiner, J. E., \& Ricci, T. V. 2014, ApJL, 796, L13

Ng, C. 2017, ArXiv e-prints, arXiv:1711.02104

Nicastro, L., Nigro, F., D’Amico, N., Lumiella, V., \& Johnston, S. 2001, A\&A, 368, 1055

Padovani, P. 2016, A\&A Rev., 24, 13

Padovani, P., Bonzini, M., Kellermann, K. I., et al. 2015, MNRAS, 452, 1263

Palliyaguru, N., Stinebring, D., McLaughlin, M., Demorest, P., \& Jones, G. 2015, ApJ, 815, 89 
Perley, R. A., \& Butler, B. J. 2013, ApJS, 204, 19

Petroff, E., Keith, M. J., Johnston, S., van Straten, W., \& Shannon, R. M. 2013, MNRAS, 435, 1610

Postman, M., Lauer, T. R., Donahue, M., et al. 2012, ApJ, 756, 159

Ramachandran, R., Demorest, P., Backer, D. C., Cognard, I., \& Lommen, A. 2006, ApJ, 645, 303

Rankin, J. M., \& Roberts, J. A. 1971, in IAU Symposium, Vol. 46, The Crab Nebula, ed. R. D. Davies \& F. Graham-Smith, 114

Reardon, D. J., Hobbs, G., Coles, W., et al. 2016, MNRAS, 455, 1751

Rickett, B. J. 1990, ARA\&A, 28, 561

Rodriguez, C., Taylor, G. B., Zavala, R. T., et al. 2006, ApJ, 646, 49

Roy, J., Gupta, Y., Pen, U.-L., et al. 2010, Experimental Astronomy, 28, 25

Scargle, J. D. 1982, ApJ, 263, 835

Shannon, R. M., Cordes, J. M., Metcalfe, T. S., et al. 2013, ApJ, 766, 5

Siemens, X., Ellis, J., Jenet, F., \& Romano, J. D. 2013, Classical and Quantum Gravity, 30, 224015

Skrutskie, M. F., Cutri, R. M., Stiening, R., et al. 2006, AJ, 131, 1163

Stinebring, D. R., \& Condon, J. J. 1990, ApJ, 352, 207 
Stinebring, D. R., Smirnova, T. V., Hankins, T. H., et al. 2000, ApJ, 539, 300

Taylor, S. R., Vallisneri, M., Ellis, J. A., et al. 2016, ApJL, 819, L6

van Straten, W., Demorest, P., \& Oslowski, S. 2012, Astronomical Research and Technology, 9, 237

Verbiest, J. P. W., Lentati, L., Hobbs, G., et al. 2016, MNRAS, 458, 1267

Vigeland, S. J., \& Siemens, X. 2016, ArXiv e-prints, arXiv:1609.03656

Volonteri, M. 2010, A\&A Rev., 18, 279

Weisberg, J. M., Nice, D. J., \& Taylor, J. H. 2010, ApJ, 722, 1030

Wheeler, J. T. 2013, Lecture Notes: Gravitational Waves, http://www.physics. usu.edu/Wheeler/GenRel2013/Notes/GravitationalWaves.pdf, ,

White, R. L., Becker, R. H., Helfand, D. J., \& Gregg, M. D. 1997, ApJ, 475, 479

Wolszczan, A., \& Frail, D. A. 1992, Nature, 355, 145

Wong, K.-W., Irwin, J. A., Yukita, M., et al. 2011, ApJL, 736, L23

Wrobel, J. M., \& Nyland, K. 2012, AJ, 144, 160

You, X. P., Hobbs, G. B., Coles, W. A., et al. 2007, MNRAS, 378, 493 